\title{
Membrane Fatty Acid Transporters as Regulators of Lipid Metabolism: Implications for Metabolic Disease
}

Citation for published version (APA):

Glatz, J. F. C., Luiken, J. J. F. P., \& Bonen, A. (2010). Membrane Fatty Acid Transporters as Regulators of Lipid Metabolism: Implications for Metabolic Disease. Physiological Reviews, 90(1), 367-417. https://doi.org/10.1152/physrev.00003.2009

Document status and date:

Published: 01/01/2010

DOI:

10.1152/physrev.00003.2009

Document Version:

Publisher's PDF, also known as Version of record

Document license:

Taverne

Please check the document version of this publication:

- A submitted manuscript is the version of the article upon submission and before peer-review. There can be important differences between the submitted version and the official published version of record.

People interested in the research are advised to contact the author for the final version of the publication, or visit the DOI to the publisher's website.

- The final author version and the galley proof are versions of the publication after peer review.

- The final published version features the final layout of the paper including the volume, issue and page numbers.

Link to publication

\footnotetext{
General rights rights.

- You may freely distribute the URL identifying the publication in the public portal. please follow below link for the End User Agreement:

www.umlib.nl/taverne-license

Take down policy

If you believe that this document breaches copyright please contact us at:

repository@maastrichtuniversity.nl

providing details and we will investigate your claim.
}

Copyright and moral rights for the publications made accessible in the public portal are retained by the authors and/or other copyright owners and it is a condition of accessing publications that users recognise and abide by the legal requirements associated with these

- Users may download and print one copy of any publication from the public portal for the purpose of private study or research.

- You may not further distribute the material or use it for any profit-making activity or commercial gain

If the publication is distributed under the terms of Article $25 \mathrm{fa}$ of the Dutch Copyright Act, indicated by the "Taverne" license above, 


\title{
Membrane Fatty Acid Transporters as Regulators of Lipid Metabolism: Implications for Metabolic Disease
}

\author{
JAN F. C. GLATZ, JOOST J. F. P. LUIKEN, AND AREND BONEN \\ Cardiovascular Research Institute Maastricht (CARIM), Maastricht University, Maastricht, The Netherlands; \\ and Department of Human Health and Nutritional Sciences, University of Guelph, Guelph, Canada
}

I. Introduction 368

II. Mechanism of Transmembrane Transport of Fatty Acids 368

A. Membrane fatty acid transport mediated by lipids or proteins? 369

B. Evidence for the involvement of membrane proteins 370

III. Membrane-Associated Fatty Acid Transporters 373

A. Plasma membrane fatty acid binding protein 373

B. Fatty acid translocase/CD36

C. Fatty acid transport proteins $\quad 375$

$\begin{array}{ll}\text { D. Caveolins } & 377\end{array}$

E. Overall conclusions on fatty acid transporters 378

IV. Functioning and Subcellular Localization of Fatty Acid Transporters 378

A. Subcellular translocation of fatty acid transporters 378

B. Posttranslational modification of fatty acid transporters 381

C. Functioning of fatty acid transporters in mitochondrial fatty acid utilization 382

D. Coordinated functioning of fatty acid transporters 383

E. Do fatty acid transporters channel fatty acids to a particular metabolic fate? 384

V. Signaling and Trafficking Events Regulating Membrane Transporter Translocation 385

A. Signaling pathways

B. Trafficking pathways $\quad 389$

VI. Chronic Physiological Regulation of Fatty Acid Transporters 391

A. Regulation of fatty acid transporter expression 391

B. Effects of development, ageing, and gender 392

C. Effects of fasting, hormones, and exercise training 393

VII. Alterations in Fatty Acid Transporters in Disease 394

A. Cardiac hypoxic disease and heart failure 394

B. Insulin resistance and type 2 diabetes 396

C. Type 1 diabetes 402

VIII. Conclusions and Perspectives 403

A. Integration of regulatory steps 403

B. Fatty acid transporters as potential therapeutic targets 404

Glatz JFC, Luiken JJFP, Bonen A. Membrane Fatty Acid Transporters as Regulators of Lipid Metabolism: Implications for Metabolic Disease. Physiol Rev 90: 367-417, 2010; doi:10.1152/physrev.00003.2009.-Long-chain fatty acids and lipids serve a wide variety of functions in mammalian homeostasis, particularly in the formation and dynamic properties of biological membranes and as fuels for energy production in tissues such as heart and skeletal muscle. On the other hand, long-chain fatty acid metabolites may exert toxic effects on cellular functions and cause cell injury. Therefore, fatty acid uptake into the cell and intracellular handling need to be carefully controlled. In the last few years, our knowledge of the regulation of cellular fatty acid uptake has dramatically increased. Notably, fatty acid uptake was found to occur by a mechanism that resembles that of cellular glucose uptake. Thus, following an acute stimulus, particularly insulin or muscle contraction, specific fatty acid transporters translocate from intracellular stores to the plasma membrane to facilitate fatty acid uptake, just as these same stimuli recruit glucose transporters to increase glucose uptake. This regulatory mechanism is important to clear lipids from the circulation postprandially and to rapidly facilitate substrate provision when the metabolic demands of heart and muscle are increased by contractile activity. Studies in both humans and animal models have implicated fatty acid transporters in the pathogenesis of diseases such as the progression of obesity to insulin resistance and type 2 diabetes. As a result, membrane fatty acid transporters are now being regarded as a promising therapeutic target to redirect lipid fluxes in the body in an organ-specific fashion. 


\section{INTRODUCTION}

The importance of long-chain fatty acids and lipids for mammalian homeostasis is well recognized. Fatty acids (for convenience this term is used to designate "longchain fatty acids," unless otherwise indicated) are primarily known as constituents of "fat," which represents a crucial and efficient energy store due to the high energy content per unit weight. Apart from their fundamental role as a fuel for energy production, fatty acids are incorporated into phospholipids forming the core of biological membranes and serve in selected signal transduction pathways to alter gene expression. However, largely due to their hydrophobic properties, fatty acids also exert harmful effects and may cause (acute) cellular injury (96, $235,468)$. Taken together, these divergent characteristics of fatty acids require that their transport among and into tissues occurs through specific mechanisms that allow their rapid and controlled distribution without the possible detrimental effects associated with their detergentlike properties.

Dietary fats typically comprise $30-40 \%$ of energy intake and consist mostly of long-chain fatty acids esterified in triacylglycerols. Lingual and pancreatic lipases will hydrolyze these triacylglycerols into monoacylglycerol and fatty acids which then are taken up by jejunal and ileal enterocytes, reesterified into triacylglycerols, and incorporated with other lipids, lipid-soluble vitamins, and apolipoproteins into chylomicrons for subsequent secretion into the circulation. Similarly, the liver secretes very-lowdensity lipoproteins produced from fatty acids synthesized de novo or taken up from blood plasma and subsequently esterified into triacylglycerols and apolipoproteins. Both chylomicrons, carrying exogenous lipids, and very-low-density lipoproteins, carrying endogenous lipids, undergo hydrolysis of their triacylglycerols by lipoprotein lipase located at the surface of the capillaries, so as to deliver the fatty acids into peripheral tissues. Fatty acids stored in adipocytes are hydrolyzed by hormone-sensitive lipase (HSL) and adipose tissue triacylglycerol lipase (ATGL), and distributed to other tissues bound to albumin via the circulation. Taken together, a complex system operates to distribute fatty acids among various tissues.

The uptake of fatty acids by parenchymal cells, especially their translocation across the cell membrane, has long been considered to occur by simple (passive) diffusion, with the rate of uptake being determined primarily by the rate of fatty acid delivery (blood flow $\times$ extracellular concentration) and the rate of intracellular fatty acid metabolism. However, from a physiological perspective, it would be highly desirable to regulate the entry of fatty acids into the cell to tune their uptake to the metabolic needs and avoid possible harmful effects of excess fatty acid accumulation. Specifically, the objective of such control would be 1) to ensure fatty acid uptake when its extracellular concentration is relatively low, 2) to limit uptake when the extracellular fatty acid concentration is relatively high, 3) potentially select for specific fatty acid types, and 4) allow rapid adjustments in fatty acid provision at the local tissue level to meet rapid fluctuations in metabolic demands, especially in heart and skeletal muscle.

In the past few decades it has become clear that various membrane-associated fatty acid-binding proteins (termed "fatty acid transporters," for convenience) facilitate the cellular entry of fatty acids, which are then accepted by cytoplasmic fatty acid binding proteins $\left(\mathrm{FABP}_{\mathrm{c}}\right)$. Furthermore, it has been found that acute changes in fatty acid uptake in response to mechanical (e.g., muscle contraction) and hormonal stimuli (insulin) are regulated by specific membrane proteins, in a fashion similar to the regulation of glucose uptake by glucose transporters. Finally, studies in both humans and animal models have implicated the membrane fatty acid transporters in various metabolic aberrations and pathologies. Thus a selective expression and/or regulation of specific (sets of) membrane-associated and cytoplasmic fatty acidbinding proteins could contribute to the control of the fatty acid uptake and utilization processes, thereby enabling tissue-specific fatty acid uptake and utilization independent of fatty acid delivery. However, while $\mathrm{FABP}_{\mathrm{c}}$ inside the cell functions as a sink for incoming fatty acids, it displays merely a permissive action in cellular fatty acid uptake in that only its full ablation reduces the rate of cellular fatty acid uptake and utilization (32, 33, 280, 370; for detailed reviews of $\mathrm{FABP}_{\mathrm{c}}$, see Refs. 142, 188, 407). In contrast, it appears that specific (sets of) plasma membrane-associated proteins are central to regulating fatty acid uptake and utilization.

In this review we discuss our current understanding of the role of membrane fatty acid transporters in cellular lipid metabolism, focusing on both the acute and chronic regulation of cellular fatty acid uptake and on chronic metabolic diseases, including myocardial disease, insulin resistance, and types 1 and 2 diabetes. Data are presented mostly for heart and skeletal muscle, as these tissues have been studied most intensively, but the concepts to be outlined generally may also apply to other tissues (see sect. VIII). Other related and recent reviews have addressed changes in lipid and carbohydrate metabolism in the failing heart (400), fatty acid metabolism in the type 2 diabetic heart (66), and skeletal muscle lipid metabolism in exercise and insulin resistance (242).

\section{MECHANISM OF TRANSMEMBRANE TRANSPORT OF FATTY ACIDS}

In recent years there has been considerable debate on the mechanism by which fatty acids are taken up by 
cells, that is, how fatty acid transport occurs across the plasma membrane, between the aqueous phases on either side of this barrier. The dispute centers around the ratelimiting kinetic step in this process, being either the adsorption of fatty acids to, or insertion into, the outer leaflet of the lipid bilayer, the subsequent transfer to the inner leaflet (referred to as flip-flop), or the desorption from the membrane into the aqueous phase (163), and whether one or more membrane proteins could facilitate either one or all of these steps or serve distinct functions in the overall uptake process. Below we discuss specific features and limitations of the methodologies and the experimental models used. The reader is referred to early $(161,163)$ and more recent $(162,233)$ reviews of the controversies concerning the rate-limiting processes involved in transferring fatty acids through the plasma membrane.

\section{A. Membrane Fatty Acid Transport Mediated by Lipids or Proteins?}

When considering the cellular uptake of fatty acids, the physical transport can be regarded as seven kinetic steps: 1) dissociation of fatty acid from extracellular albumin into the aqueous phase; 2) diffusion through the outer aqueous phase; 3) insertion into the outer leaflet of the phospholipid bilayer; 4) flip-flop from the outer to the inner leaflet, defined as the complete movement of the fatty acid across the bilayer with reorientation of the carboxyl head group from the outer lipid-water interface to the inner lipid-water interface; 5) dissociation from the inner leaflet; 6) diffusion through the inner aqueous phase; and 7 ) binding to $\mathrm{FABP}_{\mathrm{c}}$. Thereafter, the fatty acid may be activated to its acyl-CoA ester and undergo further metabolism.

The aqueous solubility of fatty acids, earlier estimated to be in the micromolar range (395), is now recognized to be extremely low, in the range 1-10 nM (465), indicating that virtually all of the fatty acids will be present in membranes or bound to proteins. The soluble fatty acid binding proteins allow fatty acids to be miscible in aqueous environments. Thus albumin in the circulation and interstitium (348) and $\mathrm{FABP}_{\mathrm{c}}$ in the cytoplasm (141, 350 ) act as extracellular and intracellular buffers, respectively, for fatty acids so that under normal physiological conditions (total fatty acid concentration in the range $100-400 \mu \mathrm{M})$ generally only $<1$ part in $10^{5}$ is present in the aqueous phase. In line with this, the average concentration of (non-protein bound) fatty acids in human plasma was reported to be $7.5 \pm 2.5 \mathrm{nM}$ (349).

Different approaches and model systems have been used to delineate the rate governing kinetic step in the overall cellular fatty acid uptake process. Various groups have studied fatty acid transport across the lipid bilayer of artificial phospholipid vesicles by incubating these vesi- cles with fatty acids, or albumin-fatty acid complexes, and monitoring either the appearance of fatty acids in the internal aqueous phase of the vesicle or the change in $\mathrm{pH}$ inside the vesicle that occurs as a result of the transmembrane movement of fatty acids. The intravesicular fatty acid concentration has been measured using ADIFAB, a fluorescent probe composed of acrylodan-derivatized intestinal type $\mathrm{FABP}_{\mathrm{c}}$ that allows the accurate assessment of very low concentrations (nM) of fatty acids in aqueous solutions without disturbing their binding equilibrium with proteins or membranes (351). ADIFAB has been trapped into phospholipid vesicles or erythrocyte ghosts during their formation and has also been microinjected into adipocytes (234). Alternatively, a pH-sensitive fluorophore such as pyranine or $2^{\prime}, 7^{\prime}$-bis(2-carboxyethyl)-5(6)carboxyfluorescein (BCECF), has been trapped inside phospholipid vesicles, to monitor the $\mathrm{H}^{+}$that dissociates from the transported (un-ionized) fatty acid upon its appearance at the inner leaflet of the bilayer (see below) (84; for review, see Ref. 59). In earlier studies transport has also been measured with fluorescently labeled fatty acid analogs $(251,408)$, but the addition of a large fluorescent moiety is expected to dramatically alter the physicochemical properties of fatty acids, and therefore alter transport rates (233). Because of these considerations, these studies will not be discussed here.

Hamilton and co-workers have monitored the movement of fatty acids across phospholipid membranes using $\mathrm{pH}$-sensitive probes. When presented either as albuminfatty acid complex, dissolved in organic solvent, or as the $\mathrm{K}^{+}$soap, the fatty acids rapidly partition into the outer leaflet of the membrane. Because in such environment the apparent $\mathrm{p} K_{\mathrm{a}}$ of the fatty acid shifts from $\sim 4.5$ in an aqueous solution to $\sim 7.6$ (independent of fatty acid type), about half of the fatty acids are present in the un-ionized, i.e., protonated, form. This uncharged species can then easily flip-flop without electrochemical restrictions to the inner leaflet of the membrane, after which a proton is donated to the interior solution and the fatty acid is available for desorption (229). Applying this approach to studies with phospholipid vesicles (230) and with adipocytes $(84,228)$ revealed linear relationships between the quantity of added fatty acids, the amount of fatty acids that binds to the plasma membrane, and the decrease in intracellular $\mathrm{pH}$. From these various studies it was concluded that binding of the fatty acid to the membrane (adsorption) occurs extremely fast, seems to be diffusionlimited, and is largely independent on the fatty acid chain length, and that transbilayer movement is fast $\left(t_{1 / 2}<1 \mathrm{~s}\right)$ for all fatty acid types and fast in cells $(\sim<10 \mathrm{~s})$. These observations have been interpreted to infer that fatty desorption from the membrane may be the rate-limiting step for the overall transport rate of fatty acids, at least for a protein-free model membrane (which represents an 
artificial situation). A more recent study has provided further evidence for this concept (383).

Kleinfeld and colleagues (97) used similar model systems to study transmembrane fatty acid transport, applying ADIFAB to detect fatty acid influx, to observe virtually identical overall rates of transport as reported by Hamilton's group (228). However, in contrast to Hamilton, they concluded that flip-flop is rate-limiting, since their data showed that the dissociation of fatty acids from the membrane is faster than flip-flop $(97,250)$. The discrepancies with other reports have been attributed to 1) the absence of albumin in some of these other studies which exposes the membranes to high $(>5 \mu \mathrm{M})$ concentrations of fatty acids that perturb the bilayer structure, and 2) to misinterpretation of the measurements (233). More recently, Kampf and Kleinfeld (232) have used quantitative fluorescence ratio microscopy to measure (noninvasively) fatty acid transport into adipocytes by imaging the intracellular (non-protein bound) fatty acid concentrations (232). Their results indicate that transport rate constants are $>50$-fold slower in adipocytes than in artificial phospholipid vesicles that contain no proteins, such as are normally present in biological membranes. From these data they conclude that fatty acid transport across adipocyte membranes is highly regulated and best described by a membrane carrier model (for review, see Ref. 233).

In summary, studies in protein-free artificial membranes show that passive flip-flop of the un-ionized form of fatty acids can occur rapidly and in a protein-independent manner across the lipid bilayer phase, indicating that the lipid bilayer does not represent a barrier for fatty acids (Fig. 1A). However, contrary to most findings with these protein-deficient, synthetic lipid vesicles, newer studies with cellular preparations that contain proteins and which apply noninvasive techniques to monitor fatty acid uptake, suggest that flip-flop is the rate-limiting step for fatty acid transport across lipid bilayers (233, 234). Because flip-flop is relatively slow and dependent on the membrane structure (being slower through the ordered phase than through the liquid-crystalline phase), diffusion rates through the lipid bilayer may not be sufficiently rapid to meet the metabolic demands of certain cells and/or under certain conditions, particularly cells in which the metabolic demands for fatty acids can be rapidly upregulated (e.g., heart and skeletal muscle). This implies that at least certain biological tissues may require membrane proteins to catalyze the flip-flop step (231). Such proteins could act as transmembrane transporters for fatty acids, but they could also attract albumin or other fatty acid carriers and enhance the concentration of fatty acids near the membrane surface, which would help overcome the barriers of the unstirred water layer. Another possibility is that membrane proteins act as a sink for fatty acids, as has been proposed for caveolin-1 which has mul- tiple basic residues at its intracellular domain that could interact with the carboxylate anion and in this way accelerate transmembrane fatty acid transport (see sect. $\mathrm{m} D$ ) (299).

A prevalent view is that both passive diffusion and protein-mediated transport contribute to the cellular uptake of fatty acids. Estimates of the contributions of these two mechanisms have been made by deconvolution of uptake curves and by the use of inhibitors of protein-mediated uptake. Because of saturation of the protein-mediated component at high fatty acid concentration, most of these studies have been interpreted to suggest that protein-mediated uptake is important at physiological concentrations of fatty acids and that passive diffusion becomes predominant at higher, presumably nonphysiological concentrations of fatty acids $(2,3)$. However, others feel that such data need to be interpreted with caution (161). Still others have questioned the coexistence of diffusional and protein-mediated fatty acid transport across the membrane's lipid phase and have proposed that fatty acid movement across the plasma membrane is primarily protein mediated $(232,233)$.

Taken together, the unifying concept arises that during the process of cellular uptake, fatty acids rapidly bind and partition into the plasma membrane, then may undergo lateral diffusion to specific domains such as lipid rafts (333) before their desorption into the intracellular compartment. Membrane proteins thus would function in regulating fatty acid entry into the cell by 1) adsorbing fatty acids from the extracellular media and modulating their transport into the membrane, and 2) segregating or organizing fatty acids for metabolism.

\section{B. Evidence for the Involvement of Membrane Proteins}

Starting in the early 1980 s, investigators from different laboratories reported that the uptake of fatty acids into various parenchymal cell types showed 1) saturation kinetics, 2) sensitivity to general inhibitors of protein-mediated plasma membrane transport processes (e.g., phloretin and proteases), 3) sensitivity to inhibition by nucleophilic fatty acid derivatives (e.g., sulfo- $N$-succinimidyloleate, SSO; later shown to specifically inhibit CD36, see sect. IvA1), and 4) sensitivity to competitive inhibition $(5,6,287,412)$. Although those observations each are in favor of protein-mediated transport, they have been disputed by others $(161,162$, 358) who argued that saturation of fatty acid transport can also be explained as saturation of metabolism in combination with passive diffusion. Moreover, the used inhibitors could theoretically inhibit fatty acid uptake via indirect effects on the structural organization of the bilayer, and the fatty acid competition experiments could unveil competition for albumin rather than for transporters. 
A

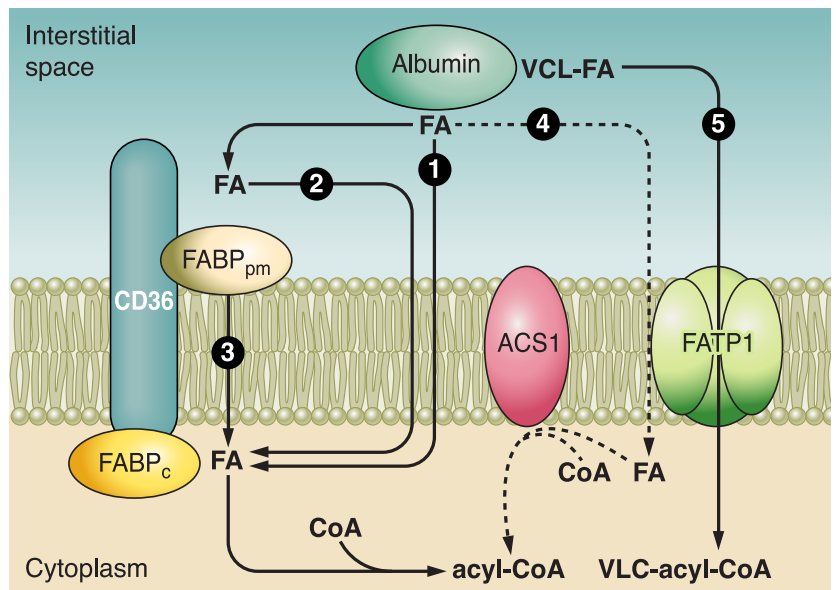

B

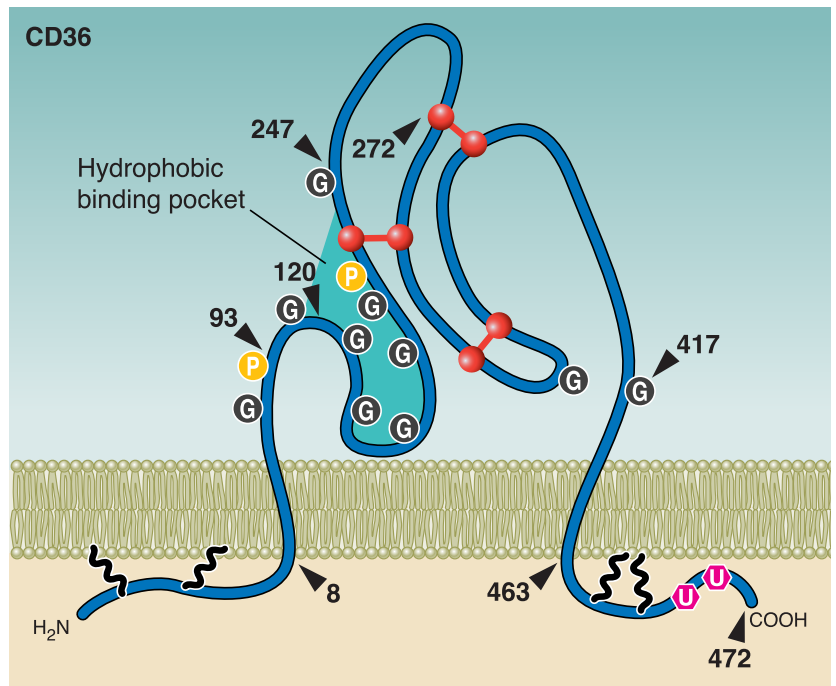

Palmitoylation site

G Glycosylation site

(U) Ubiquitination site

C

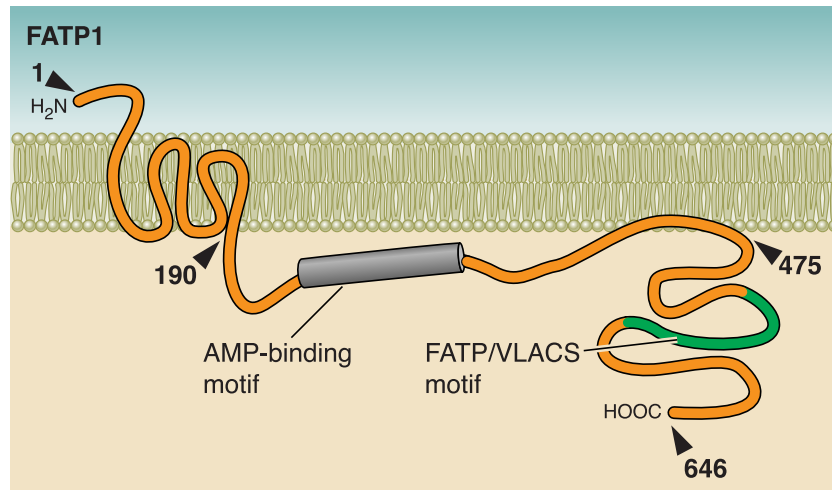

On the other hand, when (both quiescent and contracting) cardiac myocytes are subjected to increasing concentrations of externally added fatty acids, uptake becomes saturable while the intracellular fatty acid concentration remains low $(287,290)$. This observation indicates that the metabolic machinery is easily capable of trapping and metabolizing incoming fatty acids during $V_{\max }$ conditions and that saturation must come from uptake. However, during fatty acid uptake, the major portion of fatty acids is immediately esterified in both quiescent (287) and contracting (290) cardiac myocytes, implying that fatty acid uptake is closely coordinated with subsequent metabolism. For this latter reason, the introduction of giant vesicles for the study of fatty acid transport was a key methodological development $(45,286)$, as this offered for the first time the means to examine the rates of fatty acid transport in metabolically important mammalian tissues in rodents $(44,45,253,272,286)$ and in humans (48), independent of concurrent fatty acid metabolism.

FIG. 1. A: putative molecular mechanism(s) for the cellular uptake of long-chain fatty acids (FA) and of very-long-chain fatty acids (VLCFA). 1: In view of their hydrophobic nature, fatty acids could dissociate from their albumin binding sites and cross the plasma membrane by simple diffusion (referred to as uptake by passive diffusion). 2: Alternatively, membrane-associated proteins, such as the peripheral membrane protein $\mathrm{FABP}_{\mathrm{pm}}$ or the transmembrane protein CD36, could act, either alone or together, as acceptor for fatty acids to increase their concentration at the cell surface and thus enhance the number of fatty aciddiffusion events. 3: CD36 itself may also facilitate the transport of fatty acids across the phospholipid bilayer (uptake by facilitated diffusion). Once at the inner side of the cell membrane, fatty acids are bound by cytoplasmic $\mathrm{FABP}\left(\mathrm{FABP}_{\mathrm{c}}\right)$ before entering metabolic or signaling pathways. 4: Additionally, a minority of fatty acids are thought to be transported by FATP1 and rapidly activated by plasma membrane acyl-CoA synthetase (ACS1) to form acyl-Co esters. 5: VLC-FA are preferentially transported by FATP1 (or other FATPs; see text) and by action of the synthetase activity of FATP1 directly converted into VLC-acyl-CoA esters (uptake by vectorial acylation). $B$ : schematic presentation of the proposed topology of CD36 (two transmembrane domains), with the small cytoplasmic tails palmitoylated. The large extracellular loop has 10 putative $N$-linked glycosylation sites and two phosphorylation sites. Disulfide bonds between the extracellular cysteines are also shown (between amino acid residues 243-311, 272-333, and 313-323). The $\mathrm{NH}_{2}$ and $\mathrm{COOH}$ termini each contain two palmitoylation sites, and the $\mathrm{COOH}$ terminus contains two ubiquitination sites. The shaded area designates a hydrophobic pocket comprised by amino acid residues $93-183$ that likely is involved in ligand binding (residues $93-120$ was identified as the thrombospondin binding site, residues 120-155 was mapped for oxidized LDL, and residues 139-183 form a multiligand binding site). Arrowheads and numbers indicate the approximate positions of amino acid residues. [Data compiled from Tao et al. (427), Febbraio and Silverstein (125), Ibrahimi et al. (214), and Hoosdally et al. (205).] $C$ : proposed topology of FATP1. Only a short segment of the $\mathrm{NH}_{2}$ terminus faces the extracellular side of the membrane. Amino acid residues 1-190 are integrally associated with the membrane, while residues 190-257 are cytosolic and contain the AMP-binding motif that mediates the acyl-CoA synthetase activity. Amino acid residues 258-475 are peripherally associated with the inner leaflet of the cell membrane. The $\mathrm{COOH}$ terminus is located in the cytoplasm. Arrowheads and numbers indicate the approximate positions of amino acid residues. [Data compiled from Lewis et al. (266) and DiRusso et al. (103).] 
Giant vesicles are formed from parenchymal cells by incubation with an appropriate collagenase for that tissue in a high $\mathrm{K}^{+}$-containing buffer and are harvested by centrifugation $(295,332)$. These vesicles have a size $(10-15$ $\mu \mathrm{m}$ diameter) that is similar to that of small cells, are oriented $100 \%$ right-side out, and contain soluble cytoplasmic constituents such as cytoplasmic FABP which will act as a sink for fatty acids that have crossed the plasma membrane $(45,253)$. Because of the absence of subcellular organelles or metabolic enzymes, giant vesicles can be used to study substrate uptake dissected from metabolism. Studies of the rate of fatty acid entry into rat heartand skeletal muscle-derived giant vesicles have provided convincing evidence in support of the involvement of a protein-mediated system. In these vesicles, the rate of fatty acid (palmitate) entry is saturable, and inhibitable by $50-70 \%$ with protein-modifying agents (trypsin, phloretin), reactive oleate esters, antisera to putative membrane fatty acid transporters, and with other long-chain (oleate) but not short-chain (octanoate) fatty acids (45, $286,445)$. Among heart as well as red and white muscles, the $K_{\mathrm{m}}$ for vesicular fatty acid entry was similar $(6-9 \mathrm{nM})$, while the $V_{\text {max }}$ differed markedly and correlated with the fatty acid oxidation capacities of these muscles (Fig. 2). Together, these studies using rat tissue-derived giant vesicles also demonstrated that fatty acids traverse the plasma membrane in heart and skeletal muscle largely via a protein-mediated system, one that is scaled with the capacity to metabolize fatty acids in these tissues.

Although giant plasmalemmal vesicles offer the advantage of conducting substrate uptake studies in metabolically important tissues such as heart, skeletal muscle, adipose tissue, and liver from rodents (41, 44, 45, 253, 273, 286) and human skeletal muscle (48), this model system also has some limitations. For instance, specific subplasmalemmal domains may not be included in the vesicle preparation. Specifically, $\mathrm{t}$ tubules are completely excluded, while it is known that substrate transport proteins such as GLUT4 are present in t tubules $(261,264)$. In addition, caution should be taken with the interpretation of experimental data from studies with vesicles from distinct tissues. For instance, in heart and muscle, the driving gradient for net fatty acid movement is always from the extracellular space into the myocyte, while in adipose tissue fatty acid transport may be directed into or out of adipocytes. Therefore, in view of their distinct metabolic functions, muscle tissues (heart and skeletal muscle) and adipose tissue cannot serve as interchangeable model systems for examining the regulation of fatty acid transport (39).

It should be noted that the $K_{\mathrm{m}}$ for vesicular fatty acid entry into heart and skeletal muscle $\left(K_{\mathrm{m}}=6-9 \mathrm{nM}\right.$; Refs. $44,45,286)$ is similar to the extracellular or circulating concentration of (non-protein bound) fatty acids $(\sim 7.5$ $\mathrm{nM}$; see sect. ${ }_{\mathrm{II}} A$ ). Such a close relationship also exists between the transport capacities of the glucose transporter GLUT4 and the lactate transporter MCT1 with their circulating substrate concentrations in vivo, i.e., GLUT4 $K_{\mathrm{m}}=4.3 \mathrm{mM}$ (316) and plasma glucose $4-5 \mathrm{mM}$, MCT1 $K_{\mathrm{m}}=3.5 \mathrm{mM}$ and plasma lactate 1-2 mM (55). Thus, for substrate transport, it seems to be a general principle that the $K_{\mathrm{m}}$ of substrate transporters is closely matched to circulating substrate concentrations. This is advantageous, as this allows for highly sensitive protein-mediated transport of circulating substrates, including fatty acids.

In summary, there is a clear role for membrane proteins in the cellular uptake of fatty acids. Although the exact mechanism of transmembrane transport of fatty acids remains largely unknown, i.e., diffusional transbilayer movement of fatty acids in the membrane may occur independently or as part of a protein mediated process, properties of both the lipid bilayer and the fatty acid

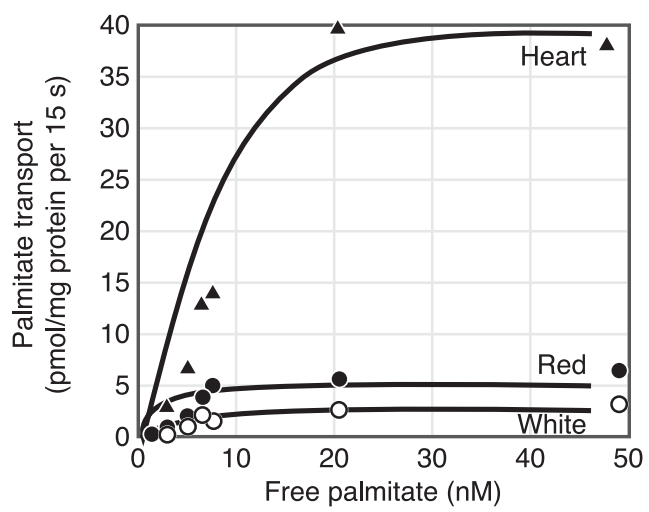

FIG. 2. Characterization of fatty acid transport into giant sarcolemmal vesicles obtained from heart and skeletal muscle. A: kinetics of palmitate transport into giant sarcolemmal vesicles from rat heart and red and white skeletal muscle. $B$ : correlation between the rate of palmitate transport into giant sarcolemmal vesicles and the amount of the fatty acid transporter CD36 located on the plasma membrane of these vesicles. $C$ : correlation between the rate of palmitate transport into giant sarcolemmal vesicles and the fatty acid oxidation capacities of the tissues. [Redrawn from studies by Bonen et al. (45), Glatz et al. (144), and Luiken et al. (286).] 
binding membrane proteins each will influence the uptake process. The presence of an intracellular fatty acid receptor beyond the cell membrane, such as $\mathrm{FABP}_{\mathrm{c}}$, is critical (280). This may explain why in certain cell lines an increased presence of fatty acid transporters in the cell membrane did not increase fatty acid uptake (118, 458, 460). Such concern does not apply to glucose uptake because of its miscibility in the cytoplasm.

\section{MEMBRANE-ASSOCIATED FATTY ACID TRANSPORTERS}

The early observations of saturation kinetics of fatty acid transport $(5,6,412)$ already triggered the search for membrane proteins that act as fatty acid transporters (172-174, 371, 413-416, 437, 438). Since then, different groups have identified integral and peripheral membrane proteins that appeared to be involved in the transport of fatty acids into parenchymal cells. For convenience, these proteins are commonly referred to as "fatty acid transporters," despite the remaining uncertainty as to the exact mechanism by which any one of these proteins participates in the transport process within the plasma membrane. Table 1 provides a listing of putative fatty acid transporters identified to date, together with their occurrence. Strikingly, these proteins differ in molecular mass and degree of posttranslational modification; some show a characteristic pattern of tissue distribution, while others are ubiquitously expressed. Interestingly, the FATPs form a fam- ily of six proteins. Support for facilitating long-chain fatty acid transport by each of these differing transporters (with the exception of caveolin-1) has been obtained from genetic studies in cell lines [plasma membrane-associated FABP $\left(\right.$ FABP $\left._{\text {pm }}\right)$ (217), CD36 (214), FATP1-6 (104, 159, 160, 268)], as well as from studies in tissues of genetically altered animals $\left[\mathrm{FABP}_{\mathrm{pm}}(85,200,315), \operatorname{CD} 36(42,86,123,153)\right.$, FATP1 (80, 479), FATP4 (315)].

\section{A. Plasma Membrane Fatty Acid Binding Protein}

In mammalian tissues, $\mathrm{FABP}_{\mathrm{pm}}$ was originally identified by Stremmel et al. in rat liver (416) and jejunal microvilli (414), and later in adipose tissue $(336,378)$ and cardiac myocytes $(393,411)$, all of which are cells with high transmembrane fluxes of fatty acids. $\mathrm{FABP}_{\mathrm{pm}}$ is peripherally bound at the outer leaflet of the plasma membrane, as $\mathrm{FABP}_{\mathrm{pm}}$ from rat liver could be isolated by a high-ionic-strength medium (394). Antibodies directed against rat liver $\mathrm{FABP}_{\mathrm{pm}}$ were found to inhibit fatty acid uptake by hepatocytes $(415,416)$, jejunal microvilli (414), adipocytes (378), cardiomyocytes (393, 411), and cardiac and skeletal muscle-derived giant vesicles $(286,445)$ by $50-75 \%$. This suggested that at least a substantial portion of overall fatty acid uptake involved the binding of fatty acids by $\mathrm{FABP}_{\mathrm{pm}}$ and that the same, or a very similar, protein is expressed in these distinct tissues. In addition, these studies do not rule out the contributions of other

TABLE 1. Membrane-associated putative fatty acid transporters

\begin{tabular}{|c|c|c|c|}
\hline Protein (Current Designation) & Molecular Mass, kDa & Tissue Occurrence & Key Reference Nos. \\
\hline $\begin{array}{l}\text { Plasma membrane fatty acid binding } \\
\text { protein }\left(\mathrm{FABP}_{\mathrm{pm}}\right)\end{array}$ & $40-43$ & $\begin{array}{l}\text { Liver, heart, muscle, adipose tissue, } \\
\text { intestine, placenta }\end{array}$ & $\begin{array}{l}26,63,85,217,378,411,414,416 \\
\quad 419\end{array}$ \\
\hline FA transport protein (FATP) & 63 & & \\
\hline FATP1 & & $\begin{array}{l}\text { Adipose tissue, heart, muscle, brain, kidney, } \\
\text { skin, lung }\end{array}$ & $181,185,191,219,315,371,375$ \\
\hline FATP2 & & Kidney, liver, intestine & $183,190,191,226,366$ \\
\hline FATP3 & & Lung, liver, testis, skin & $191,328,375$ \\
\hline FATP4 & & $\begin{array}{l}\text { Intestine, brain, kidney, liver, skin, lung, } \\
\text { heart, skeletal muscle }\end{array}$ & $\begin{array}{l}107,153,181,185-187,191,219 \\
\quad 315,375\end{array}$ \\
\hline FATP5 & & Liver & $105,191,209$ \\
\hline FATP6 & & $\begin{array}{l}\text { Heart, skeletal muscle, placenta, testis, } \\
\text { adrenal glands, kidney, bladder, uterus, } \\
\text { skin }\end{array}$ & $139,181,219,375$ \\
\hline Fatty acid translocase/CD36 & $88^{*}$ & $\begin{array}{l}\text { Heart, intestine, skeletal muscle, adipose } \\
\text { tissue, spleen, platelets, } \\
\text { monocyte/macrophage, endothelium, } \\
\text { epidermis, kidney, brain, liver }\end{array}$ & $4,124,150,272,273,294,375,459$ \\
\hline Caveolin-1 & $21-24$ & $\begin{array}{l}\text { Ubiquitously expressed, except in muscle } \\
\text { and heart where caveolin-3 is } \\
\text { predominantly expressed }\end{array}$ & $333,335,436$ \\
\hline
\end{tabular}

The designation "fatty acid transporter" is used for convenience but does not necessarily imply a classic transmembrane transport mechanism such as that of GLUT4. The proteins share the feature of facilitating the transmembrane translocation of (long-chain) fatty acids, although the role of caveolins in fatty acid transport remains controversial (see sect. $\mathrm{III} D$ ). Tissue occurrence refers to tissues for which the expression (mRNA or protein) was reported. Relative occurrence is not always clear, as this may differ across species and will depend among other things on developmental and nutritional status, muscle and cardiac activity, and health status. *Glycosylated protein mass. The mass of the nonglycosylated protein is $53 \mathrm{kDa}$. Glycosylation may differ in some tissues, such as mammary gland, where CD36 molecular mass is $\sim 75 \mathrm{kDa}$. 
fatty acid binding proteins, as inhibition of fatty acid transport by $\mathrm{FABP}_{\mathrm{pm}}$ antibodies was incomplete.

Analysis of its amino acid sequence showed $\mathrm{FABP}_{\mathrm{pm}}$ to be identical to the mitochondrial aspartate aminotransferase (mAspAt) $(26,419) . \mathrm{FABP}_{\mathrm{pm}}$ and mAspAt are derived from the same gene while not requiring alternative splicing of the mRNA (51). Apparently, $\mathrm{FABP}_{\mathrm{pm}} / \mathrm{mAspAt}$ is a protein with distinct functions at different subcellular sites. In Xenopus laevis oocytes (484), 3T3 fibroblasts (217), and rat skeletal muscle $(85,200)$, transfected with mAspAt cDNA, $\mathrm{FABP}_{\mathrm{pm}} / \mathrm{mAspAt}$ was localized both to the mitochondria (200) and to the plasma membrane $(85,200$, $217,484)$. This increased the rates of fatty acid transport into giant sarcolemmal vesicles obtained from skeletal muscle $(85,200)$. However, the relative increase in plasmalemmal $\mathrm{FABP}_{\mathrm{pm}}(+173 \%)$ was far in excess of the increase in fatty acid transport (+79\%) (85), suggesting that the transport capacity of $\mathrm{FABP}_{\mathrm{pm}}$ alone is perhaps modest.

\section{Effects of $F A B P_{p m}$ on fatty acid metabolism}

$\mathrm{FABP}_{\mathrm{pm}}$ overexpression in skeletal muscle did not alter triacylglycerol formation, but fatty acid oxidation was increased $(85,200)$. This was attributable to additional fatty acids transported into the muscle cell, as the concurrent overexpression of $\mathrm{FABP}_{\mathrm{pm}} / \mathrm{mAspAt}$ in mitochondria did not alter fatty oxidation by isolated mitochondria (200; see also sect. Iv $B$ ). There is some evidence for cooperation of $\mathrm{FABP}_{\mathrm{pm}}$ with other fatty acid transporters, specifically CD36 (see sect. IVA) (75). Ablation studies of $\mathrm{FABP}_{\mathrm{pm}} / \mathrm{mAspAt}$ have not yet been performed.

\section{B. Fatty Acid Translocase/CD36}

Studies by Abumrad and co-workers on the inhibitory action of reactive sulfo- $N$-succinimidyl esters of longchain fatty acids (SSO, see sect. ${ }_{\mathrm{I}} B$ ) on fatty acid uptake by rat adipocytes (172-174) led to the identification of an integral membrane protein designated (putative) membrane fatty acid translocase (4) that appeared identical to leukocyte cluster-of-differentiation antigen CD36 (glycoprotein IV), now recognized as a class B scavenger receptor protein with multiple functions, particularly the binding of thrombospondin, oxidized low-density lipoprotein (LDL), and anionic phospholipids, and its action as a gustatory lipid sensor $(124,125,212,241,417,420)$. CD36 is a 472 -amino acid (53 $\mathrm{kDa})$ protein that has a hairpin membrane topology with two transmembrane spanning regions, with both the $\mathrm{NH}_{2}$ and $\mathrm{COOH}$ termini as short segments in the cytoplasm (427) (Fig. $1 B$ ). The $\mathrm{NH}_{2}$ terminal hydrophobic domain appears to serve as a transmembrane anchor (427). The protein is heavily glycosylated (10 predicted $N$-linked glycosylation sites situated in the large extracellular loop), has two phosphorylation sites (Thr-92 and Ser-237) and three external disulfide bridges, and contains four palmitoylation sites (225, 427), two each at the extreme $\mathrm{NH}_{2}$ and $\mathrm{COOH}$ termini (cysteines 3, 7, 464, and 466) (see sect. IvB1). The COOHterminal domain also contains two ubiquitination sites (Lys-469 and Lys-472) (see sect. IVB3). Studies in rat hepatoma cells suggest that the COOH-terminal YCAR motif is required for CD36 localization to the cell surface and to enhance long-chain fatty acid uptake (119).

The extensive glycosylation increases the apparent mass of CD36 from 53 to $88 \mathrm{kDa}$ (Table 1). However, it was recently shown that mature glycosylation is not necessary for surface expression of CD36 in mammalian cells (205). CD36 is associated with the cholesterol- and sphingolipid-rich membrane microdomains known as rafts (or as caveolae when they contain caveolin). The role of caveolae in CD36 functioning is discussed in section $\mathrm{III} D$. Whether caveolins are involved in targeting CD36 to the plasma membrane is not entirely clear given some contradictory evidence among studies in the heart $(14,15)$, mouse embryonic fibroblasts (354), and 3T3-L1 fibroblasts and adipocytes $(333,334)$. Alternatively, palmitoylation of each of the cytoplasmic tails could help recruit CD36 to these membrane microdomains. In this respect, palmitoylation is often involved in regulation of intracellular trafficking and localization of membrane proteins (reviewed in Ref. 149), especially in the trafficking of these proteins to lipid rafts and/or caveolae.

CD36 is ubiquitously expressed in many tissues, as well as endothelial cells, platelets, and macrophages, and is involved in angiogenesis, atherosclerosis, inflammation, and lipid metabolism (for review, see Refs. 124, 125). In vitro studies showed that CD36 purified from adipose tissue binds long-chain but not short-chain fatty acids (16, 225). A key role for CD36 in fatty acid transport was demonstrated when Ob17PY fibroblasts (214), C2C12 fibroblasts (20), and skeletal muscle (315) were transfected with CD36, which resulted in increased rates of fatty acid uptake. However, others have found that transfecting CD36 into CHO cells fails to increase fatty acid uptake under basal conditions $(118,460)$ or when insulin was used to increase plasmalemmal CD36 (460), suggesting that in these cells, such an increase requires additional proteins, or a protein activation step. CD36 is also required both for the uptake of very-long-chain fatty acids (VLCFAs) in cultured cells and for the intestinal absorption of dietary VLCFAs in mice (110). CD36 can also function as a cell adhesion molecule or as a class B scavenger receptor $(124,125)$. Finally, CD36 appears to be a selective and nonredundant sensor of microbial diacylglycerides (192). This CD36 versatility for a wide variety of ligands may possibly be due to differences in the glycosylation of the protein and/or specific interaction with other proteins or membrane constituents. 


\section{Effects of CD36 on basal fatty acid metabolism}

Under basal conditions, a null mutation in murine CD36 reduced the uptake of the fatty acid analogs 15-( $p$ iodophenyl)-3-(R,S)-methyl pentadecanoic acid (BMIPP) and 15 -( $p$-iodophenyl)pentadecanoic acid (IPPA) in vivo in heart ( -50 to $-80 \%)$, skeletal muscle ( -40 to $-75 \%)$, and adipose tissue $(-60$ to $-70 \%)(86)$. CD36 null mice did not show alterations in fatty acid uptake by the liver (86), an organ with absent or very low CD36 expression $(123,253,272)$. Comparable reductions were also observed in studies using a naturally occurring fatty acid (palmitate) in CD36 null skeletal muscles (-23\%) (42), but not in CD36 null cardiac myocytes (153), presumably due to the compensatory twofold overexpression of FATP1 in CD36 null cardiac myocytes (153).

In perfused, CD36-null murine muscles, the basal rates of fatty acid oxidation (-26\%) and triacylglycerol formation $(-38 \%)$ were reduced (42). Similarly, in CD36 null cardiac myocytes, there was a $25 \%$ reduction in fatty acid oxidation (216), which was restored by transgenic rescue of CD36 (216). There were also reductions in intracellular triacylglycerol concentrations in perfused CD36 null hearts ( -27 to $-64 \%$; Refs. 86, 216) and in skeletal muscle ( $-70 \%$; Ref. 86$)$. This was likely due to a reduction in the basal rate of triacylglycerol esterification in CD36 null mice ( $-38 \%$, in red muscles; Ref. 42). In contrast to these studies in CD36 null mice, no changes in basal rates of fatty acid metabolism were observed in isolated soleus muscles of CD36-overexpressing mice (213), presumably since rates of fatty acid metabolism are low in isolated muscle that are at complete rest (see below).

\section{Effects of CD36 on fatty acid metabolism during metabolic challenges}

Examining the role of CD36 on fatty acid metabolism under basal conditions has underestimated its role in regulating fatty acid uptake and metabolism. Metabolic challenge studies have now been performed in isolated muscles of CD36 overexpressing mice (213) and in hindlimb muscles (42), hearts (258), and cardiac myocytes (153) of CD36 null mice, using stimuli that are known to increase triacylglycerol formation (insulin, Refs. 91, 115, 278 ) and/or fatty acid oxidation (contraction, AICAR, oligomycin, working heart, Refs. 213, 277, 385, 386), as well as inducing the translocation of CD36 to the plasma membrane in these tissues $(44,73,91,277,278)$ (see sect. Iv $A$ ).

In cardiac myocytes and in perfused skeletal muscles of CD36 null mice, stimulation of fatty acid uptake by oxidation-enhancing agents was markedly impaired [cardiac myocytes: wild type (WT) $+150 \%$; knock out (KO) $+20 \%$ (153); skeletal muscle: WT +77\%, KO +13\% (42)], while insulin stimulation of fatty acid uptake was also markedly impaired in muscle of CD36 null mice $(+21 \%)$ compared with WT mice $(+60 \%)$ (42). These reductions in fatty acid uptake also contributed to altered rates of fatty acid metabolism. For example, in working hearts, fatty acid oxidation remained 40-60\% lower in CD36 null than in WT mice $(254,258)$, and in CD36 null muscle, AICARstimulated fatty acid oxidation was also markedly impaired (KO +38\%; WT + 100\%; Ref. 42). Conversely, in CD36 overexpressing mice, muscle contraction increased fatty acid oxidation by $+400 \%$, while in WT mice, the increase was much less, i.e., $+100 \%$. Insulin-stimulated triacylglycerol esterification in CD36 null red muscle $(+34 \%)$ was increased much less than in red muscles of WT mice $(+70 \%)$ (42). Since the cellular signaling pathways and enzymatic activities are not altered in CD36 null mice $(42,86,153)$, these studies demonstrate that CD36 contributes markedly to the regulation of fatty acid oxidation and esterification in heart and skeletal muscle, particularly during metabolic challenges.

\section{Fatty Acid Transport Proteins}

Schaffer and Lodish (371) used an expression cloning strategy to identify a protein that, when expressed in COS7 cells, increased the uptake of fluorescently labeled fatty acids. This 646-amino acid fatty acid transport protein (FATP; recently renamed as FATP1) is an integral membrane protein that has six membrane-spanning regions. Subsequently, others disclosed the existence of a family of integral membrane FATPs, referred to as FATP2-6 (139, 191). These FATP isoforms are expressed in somewhat of a tissue-specific manner (Table 1) (107, 469).

FATP1 contains a hydrophobic $\mathrm{NH}_{2}$-terminal region (residues 1-190) that is integrally associated with membranes, whereas amino acid residues 258-313 and 314475 are only peripherally membrane associated, and residues 191-257 and 476-646 do not direct membrane association and likely face the cytosol (266) (Fig. 1C). The mechanism of action for FATP1 involves ATP binding that is dependent on serine-250 of the IYTSGTTGXPK motif (residues 247-257) $(347,418)$. FATP1 may also form detergent-resistant dimers that have a functional role in fatty acid transport (347). The region between amino acid residues 191-475 is sufficient to allow an association of FATP1s (347). Both the monomeric $(\sim 63 \mathrm{kDa})$ and oligomeric forms $(\sim 130 \mathrm{kDa})$ are present in NIH 3T3 cells (347).

FATP1 is expressed in many tissues, including brain, kidney, lung (191), skin (375), adipose tissue (107), heart $(73,80,107,139,153,181)$, and skeletal muscle (42, 46, $107,219,315$ ) (Table 1). FATP2 is expressed primarily in liver and kidney $(183,191,226,366)$ as well as in the intestines (190), whereas FATP3 has a more restricted distribution being expressed in liver, lung, testis, and skin 
(191, 328, 375). FATP4 is expressed in intestine, brain, kidney, liver, skin, lung, heart, and skeletal muscle (107, 153, 181, 185-187, 191, 219, 315, 375). In contrast, FATP5 is only expressed in liver $(105,191,209)$. Finally, although FATP6 was deemed to be heart specific (139), this protein is expressed in a number of other tissues, including skeletal muscle, placenta, testis, adrenal glands, kidney, bladder, uterus, and skin (139, 181, 219, 375).

\section{Fatty acid transport roles of FATPs}

Expression of the murine FATPs in a genetically defined yeast strain increased fatty acid transport with varying degrees of effectiveness. FATP1, -2 , and -4 are particularly effective in facilitating the rates of long-chain fatty acid transport by 8.2-, 4.5-, and 13.1-fold, respectively, whereas FATP3 and -5 provide only a modest 2 -fold increase, and FATP6 provides virtually no increase in long-chain fatty acid transport (104). However, studies in other models suggest somewhat different fatty acid transport roles for FATPs. In MA-10 Leydig cells, FATP3 expression failed to alter fatty acid transport (328). Knockdown studies of FATP1 (3T3-L1 adipocytes) revealed that basal fatty acid uptake was reduced (268), whereas with FATP4 knockdown (3T3-L1 adipocytes) or overexpression (HEK-293 cells), there were no changes in fatty acid uptake (268). Another study has indicated that FATP4 is not present at the plasma membrane and is not a fatty acid transporter in a wide variety of cultured cells (300). In contrast, experiments in rat skeletal muscle indicated that FATP1 and -4 have fatty acid transport function, with FATP4 being more effective than FATP1 (315), as was also observed in yeast (104). Others have shown that the transport capacity of FATPs in 293 cells depends on the fatty acid used (palmitate transport: FATP6 $>>$ FATP1 $>$ FATP4; oleate transport: FATP4 $>$ FATP1 $=$ FATP6) (139), suggesting that different FATPs transport specific fatty acids with different efficiencies. Thus the claim that FATP6 is the most important fatty acid transporter in the heart (139) is premature. Interestingly, in vitro FATP5 from liver exhibits both fatty acid transport and bile acidCoA ligase activity $(104,107,191,209)$.

The differing transport capacities for the same FATP isoform among several studies may indicate that there may well be cell-type specific responses, whereby the functioning of the FATP isoforms may depend on the presence and/or interaction with other proteins or membrane constituents. Therefore, some care is required when using cultured cell lines to extrapolate the function of FATPs and other fatty acid transporters to the in vivo situation in mammalian tissues, as all the necessary biochemical transport and/or trafficking machinery normally present in mammalian tissues may be incomplete in selected cell lines, as has been suggested in several recent studies $(118,460)$.
To elucidate the in vivo roles of the FATPs, generation of transgenic mice has been undertaken for FATP1, -4 , and -5 . Cardiac-specific overexpression (8-fold) of FATP1 increased the rate of fatty acid uptake (4-fold) (80). In contrast, ablation of FATP1 did not reduce basal rates of fatty acid uptake, either in adipocytes or in skeletal muscle (479). Combined, these findings may indicate that FATP1 is a functionally important fatty acid transporter but only at nonphysiologically high expression levels, and that at normal expression levels it contributes minimally to the bulk uptake of fatty acids in muscle tissues. Nevertheless, during metabolic challenges, the role of FATP1 might become more important, as it was reported that insulin-stimulated fatty acid uptake is markedly reduced in FATP1 null muscle (479). Deletion of one allele of FATP4 resulted in 48\% reduction of FATP4 protein levels and a $40 \%$ reduction of BODIPY-fatty acid uptake by isolated enterocytes, but did not affect intestinal fat absorption either with normal diet or with a highfat diet (138). Deletion of both FATP4 alleles resulted in early embryonic lethality $(138,187)$, possibly as a result of reduced fat absorption in association with FATP4 deletion from the epithelial cells of the visceral endoderm and the brush-border membrane of extraembryonic endodermal cells, as well as neonatally lethal restrictive dermopathy (187). In epidermal keratinocytes, FATP4 is essential for the maintenance of a normal epidermal structure (186). FATP5 null mice showed a decreased fatty acid uptake rate $(-50 \%)$ in primary hepatocytes and reduced hepatic fatty acid and triacylglycerol contents (105). Knockdown of hepatic FATP5 in vivo resulted in a marked reduction of hepatic dietary fatty acid uptake, reduced caloric uptake, and concomitant protection from diet-induced nonalcoholic fatty liver disease (106). Defective bile acid conjugation, which relates to the bile acidCoA synthetase activity of FATP5, has been observed in FATP5 null mice (209).

\section{Very-long-chain acyl-CoA synthetase activity of FATPs}

In early studies on FATPs, it was found that these proteins shared considerable sequence homologies and domain organization to acyl-CoA synthetases, suggesting that FATPs are members of the superfamily of adenylate forming acyl-CoA synthetases, particularly very-longchain acyl-CoA synthetases (VLACS) for >C22 (120, 371, $470,471)$. However, in vivo very-long-chain fatty acids constitute only a small fraction of the total fatty acid pool. For example, in dogs fatty acids $\geq \mathrm{C} 22$ constitute $0.5 \%$ of the circulating fatty acids and $1.8 \%$ of the triacylglycerol fatty acids (456). Circulating fatty acids and triacylglycerols are largely comprised ( $\geq 90 \%)$ of long-chain (C16:0C18:2) fatty acids in dogs (456) and rodents (145), and this is also reflected in the makeup of intramuscular lipids 
$(145,456)$. These observations would seem to imply that very-long-chain fatty acids are relatively unimportant substrates for cardiac and skeletal muscle energetics and that the VLACS function of FATP1 would not mediate the transport function for long-chain (C14:0-C18:1) fatty acids. Indeed, there is only a limited sequence similarity between FATP1 and the multigene family of long-chain acyl-CoA synthetases (ACS) for these abundant longchain (C14:0-C18:1) fatty acids. Nevertheless, the concern remained that the fatty acid transport function of FATP was due to the rapid esterification of fatty acids to CoA thioesters by acyl-CoA synthetase (a process known as vectorial acylation; Refs. 249, 323), as this would serve to increase the fatty acid concentration gradient across the plasma membrane by removing fatty acids once they have traversed the plasma membrane. A similar process of metabolic trapping by the hexokinase-catalyzed phosphorylation of glucose has long been recognized. Although the overexpression of FATPs increases the activity of long-chain and very-long-chain fatty acyl-CoA synthetases $(87,120,185,448,486,487)$, it is doubtful that VLACS activity accounts for the transport of long-chain fatty acids $(159,160,486)$ because for the most abundant circulating fatty acids (C:14-C:18), the acyl-CoA synthetase activity is either very low (FATP1), or alternatively, in vivo, these fatty acids are not preferred (FATP4).

\section{Long-chain acyl-CoA synthetases}

Expression of fatty acyl-CoA synthetases that are unrelated to the FATP-associated VLACS can enhance fatty acid uptake in Escherichia coli $(292,373,472)$, yeast (432), and 3T3-L1 adipocytes $(136,346)$, suggesting that vectorial acylation may drive fatty acid transport. In 3T3-L1 adipocytes, ACS1 is an integral membrane protein which colocalizes and interacts with FATP1 $(136,346)$. Overexpression of each of these two proteins increases fatty acid uptake, and their concomitant overexpression has a synergistic effect on fatty acid uptake (136), while blocking ACS1 activity reduces fatty acid uptake (346). However, these studies (136, 292, 346, 373, 432, 472) should not be taken to imply that the formation of fatty acyl-CoAs (vectorial acylation) obviates the need for a fatty acid transport mechanism. Instead, fatty acid transport and ACS1 activity are most likely complementary processes, comparable to that of GLUT4-facilitated glucose transport and hexokinase II activity in vivo (130135). Indeed, in skeletal muscle, the coordinated expression of CD36 and ACS1 in red and white muscles, and in chronically stimulated muscles $(r=0.98)$ is an example of functional complementarity between these proteins, as this allows a greater rate of fatty acids to be transported into the muscle cell via CD36, which can then be activated by ACS1 (279).
The independence of fatty acid transport from vectorial acylation has been shown in a number of studies. First, with giant vesicles there is no evidence for any fatty acid metabolism, because incoming fatty acids were retrieved as fatty acids in the vesicular lumen, most likely bound to cytoplasmic FABP (45, 286). Second, studies by DiRusso et al. (104) have resolved some of the confusion concerning the fatty acid transport functions and acyl-CoA synthetase activities of FATPs. Expression of the murine FATP1-6 in a genetically defined yeast strain, which normally cannot transport longchain fatty acids, and which has a reduced VLACS activity, demonstrated the independent fatty acid transport capacities from the VLACS activity of many of these FATP isoforms. Specifically, FATP1-5 expression, but not FATP6, increased fatty acid transport differentially, i.e., 2.3-fold (FATP3 and -5), 4.4-fold (FATP2), 8-fold (FATP1), and 13fold (FATP4). Concomitantly, these proteins only marginally altered oleoyl-CoA synthase activity (104) while, except for FATP5, VLACS activity was increased for all other FATPs (range 2- to 24-fold; Ref. 104). Thus FATP isoforms appear to play unique roles in fatty acid trafficking, including the transport of exogenous long-chain fatty acids (FATP1-5) and very-long-chain fatty acid activation (FATP1-4, -6). Importantly, these studies also demonstrated that the fatty acyl activation function of FATPs is not required for long-chain (C:14-C:18) fatty acid transport (104).

In summary, it appears that the fatty acid transport function of FATPs is not attributable to their FATP-associated VLACS activity, which is very low compared with ACS1 activity. Moreover, ACS1 activity by itself also does not account for fatty acid transport. Instead, in vivo, FATPs, and likely other fatty acid transporters, act in a concerted fashion with ACS1 as functional complex, to take up and activate fatty acids, thereby maintaining an effective transplasmalemmal fatty acid gradient and coupling fatty acid transport to its metabolism.

\section{Caveolins}

Caveolins are the defining protein constituents of caveolae, which are specialized microdomains of the plasma membrane, enriched in cholesterol, sphingomyelins, and signaling and receptor proteins (88, 327). Caveolins are responsible for the invagination of the plasma membrane, giving the caveolar microdomains their flask-shaped appearance. They are small integral membrane proteins $(\sim 22 \mathrm{kDa})$ with an additional hydrophobic scaffolding domain for binding to other proteins. Currently, three members of the caveolin family have been identified. Caveolin- 1 and -2 have a relatively ubiquitous distribution pattern in mammalian tissues with the exception of muscle tissues, whereas caveolin-3 is the predominant isoform in muscle and heart $(88,148)$.

Unexpectedly, a screen for high-affinity fatty acid binding proteins within adipocyte plasma membranes us- 
ing photoreactive fatty acid analogs yielded a single protein of $22 \mathrm{kDa}$, subsequently identified as caveolin-1 (436). This raised the notion that next to its caveolar-related functions, caveolin-1 could serve as a fatty acid transporter.

Studies by Pohl and colleagues $(333,335)$ on the role of caveolae in the uptake of fatty acids in HepG2 and 3T3 cells using cholesterol-depleting agents, caveolin-1 antisense oligonucleotides or caveolin dominant-negative mutants revealed an up to $50 \%$ inhibition of fatty acid uptake by these treatments. Interestingly, caveolae also contain CD36, suggesting that CD36 is involved in caveolae-mediated fatty acid uptake (333). This notion gained further credence by the observation that caveolin-1 ablation in fibroblasts reduced the plasma membrane content of CD36 in parallel with a reduction of cellular fatty acid uptake (354). Conversely, caveolin-1 overexpression redirected CD36 to the plasma membrane and rescued fatty acid uptake (354). Whereas the initial experiments with photoreactive fatty acid analogs revealed caveolin- 1 to be a potential fatty acid transporter (436), the latter experiments (354) indicate that caveolins function in fatty acid uptake in an indirect manner, i.e., by offering plasma membrane docking sites for CD36.

It should be noted, however, that this CD36-assisting function of caveolin-1 (and -3) was questioned in other studies, because 1) overexpression of caveolin-1 is able to modulate fatty acid uptake in HEK-293 cells which do not express CD36 (299, 382), 2) cholesterol depletion in adipocytes reversibly inhibited fatty acid uptake without altering the cell surface localization of caveolin-1 or CD36 (95), and 3) in hearts of caveolin-1 null mice in vivo, there was an increase in fatty acid uptake $(+47 \%)$ (15). These conflicting conclusions about the cooperation between caveolin-1 and CD36 in cellular fatty acid uptake may relate to tissue-specific or cell-specific differences, or may be due to the different methods and/or the model systems used. Hence, fine-tuning of these studies is needed to assess the role of caveolin-1 in cellular fatty acid uptake.

With respect to caveolin-3, the predominant isoform in muscle and heart, this protein was found to be colocalized with CD36 at the sarcolemma $(236,464)$. While this colocalization suggests that in muscle tissues caveolin-3 might assist CD36 in fatty acid uptake, studies in hearts of caveolin-3 knockout mice did not reveal any changes in cardiac fatty acid uptake and metabolism (14). Taken together, the functioning of members of the caveolin family as fatty acid transporters is still controversial.

\section{E. Overall Conclusions on Fatty Acid Transporters}

Genetic studies in vitro and/or in vivo have greatly helped in establishing the roles of $\mathrm{FABP}_{\mathrm{pm}}$, CD36, FATPs, and caveolins in cellular fatty acid uptake. One remarkable issue is that all fatty acid transporters also appear to have functions that are unrelated to fatty acid transport; for example, $\mathrm{FABP}_{\mathrm{pm}}$ and the FATPs contain mAspAt activity and VLACS activity, respectively; CD36 displays multiple other functions, including thrombospondin binding; caveolins possess the ability to form caveolar regions. This notion should not be taken to preclude a significant role for these proteins in the regulation of fatty acid flux across the plasma membrane. Importantly, there are numerous unresolved issues concerning the fatty acid transporters: 1) their three-dimensional protein structure and membrane topology; 2) their specific mode of action including possible interactions with each other and with other proteins; 3) their possible substrate specificity, i.e., the relative affinity towards saturated versus unsaturated and polyunsaturated fatty acid species; and 4) their ability to couple fatty acid uptake to channeling into distinct metabolic pathways (see sect. $\mathrm{r} D$ ). Moreover, the listing of fatty acid transporters may not be complete, as suggestions have been made that other fatty acid proteins may yet be identified (cf. Ref. 234). Finally, as mentioned above (see sect. II $B$ ), caution should be taken by using any one cell type or mammalian tissue as a universal model for fatty acid transport or transporters. In view of the distinct metabolic roles of selected tissues and differences in bidirectional (adipose tissue) and monodirectional (heart, muscle, liver) transmembrane fatty acid fluxes, the expression and functioning of the various fatty acid transporters may well be quite dissimilar.

\section{FUNCTIONING AND SUBCELLULAR LOCALIZATION OF FATTY ACID TRANSPORTERS}

The studies reviewed in the previous section have established that a number of distinct membrane proteins facilitate the transport of fatty acids across the plasma membrane. Insight into the functioning and physiological significance of these fatty acid transporters was obtained from studies in which it was shown that fatty acid transporters can traffic between intracellular depots, and the plasma membrane and mitochondria in response to physiological perturbations (muscle contraction, exercise, insulin), and from studies on posttranslational modification of fatty acid transporters, as will be outlined below.

\section{A. Subcellular Translocation of Fatty Acid Transporters}

The observation that muscle-specific overexpression of CD36 increased fatty acid uptake only during muscle contraction (213) triggered us to investigate the possibil- 
ity that in response to muscle contraction CD36 would translocate from a putative intracellular storage site to the sarcolemma to increase fatty acid uptake. Such a mechanism would resemble the well-known regulation of muscle glucose uptake by subcellular translocation of GLUT4 (for review, see Refs. 99, 208, 362, 365, 428).

\section{Contraction-mediated regulation of fatty acid transport and transporters}

The first evidence that fatty acid uptake was regulated acutely at the level of the plasma membrane was obtained from studies in which the metabolic demands of skeletal muscle in vivo were increased via electrically induced contraction (44). Almost immediately with the onset of muscle contraction there was an increase in the rate of fatty acid transport into giant sarcolemmal vesicles prepared from these muscles (+20-29\% after 1-5 $\mathrm{min})$ with a maximal 1.8-fold increase being attained after $30 \mathrm{~min}$. Upon cessation of muscle contraction for $20 \mathrm{~min}$, rates of fatty acid transport returned to basal rates. The contraction-induced increases in fatty acid transport rates were linearly related with the intensity of the muscle contraction, indicating that fatty acid uptake into muscle scaled with the muscles' metabolic demands. These studies also revealed for the first time that the contractioninduced increase in fatty acid transport was accompanied by a concurrent translocation of a fatty acid transporter, CD36, from an intracellular depot to the plasma membrane (1.4-fold) and the reinternalization of this transporter with the cessation of muscle contraction (Fig. 3) (44). All this was highly reminiscent of contraction-stimulated GLUT4 translocation and glucose transport described a decade earlier (for review, see Ref. 362). Since the contraction-induced fatty acid transport was inhibited by the specific CD36 inhibitor SSO, which binds covalently to CD36 (44, 92, 153), a central physiological role for this fatty acid transporter was established (Fig. 4). In subsequent studies in cardiac myocytes, it was also shown that contraction induced the translocation of CD36 from an endosomal depot to the plasma membrane (277).

Since these initial studies, others have confirmed that contractile activity increases fatty acid transport via the translocation of CD36 in skeletal muscle (443). Contraction can also induce the translocation of $\mathrm{FABP}_{\mathrm{pm}}$ in skeletal muscle $(164,219)$, as can AICAR-induced AMPK activation in the heart (73). In addition, studies in cell lines have confirmed, using cell surface labeling techniques, that CD36 translocation is a rapid and reversible process $(118,325,460)$. Recently, we have also found that muscle contraction increases the content of FATP1 and FATP4 at the plasma membrane, while plasmalemmal FATP6 is not altered (219). Nevertheless, CD36 is fundamental to facil-

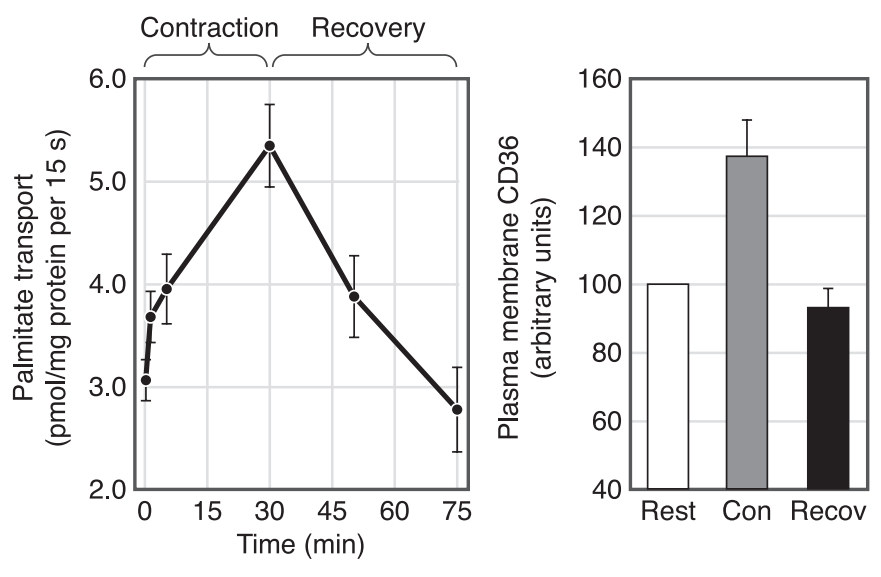

FIG. 3. Acute regulation of fatty acid uptake involves the cellular redistribution of CD36. Left: fatty acid uptake into giant sarcolemmal vesicles at selected time points during muscle contraction. Rat hindlimb skeletal muscles were electrically stimulated via the sciatic nerve to contract for 1, 5, and $30 \mathrm{~min}$. Thereafter, muscle recovered from contraction for 20 and $45 \mathrm{~min}$. At each time point, giant sarcolemmal vesicles were prepared and fatty acid uptake determined. Right: amount of sarcolemmal CD36 determined by Western blotting in resting muscle, after $30 \mathrm{~min}$ of muscle contraction, and after 45 min recovery. [Data from Bonen et al. (44).]

itating the increase in fatty acid transport, since the contraction- or oligomycin-induced increase in fatty acid transport rate is completely blunted in skeletal muscle $(44,199,219)$ and cardiac myocytes (153) by SSO and by sulfo- $N$-succinimidylpalmitate (SSP), another specific inhibitor of CD36. Moreover, contraction-induced fatty acid transport is lost in cardiac myocytes from CD36 knockout mice (153) and is only minimally increased by the contraction mimetic agent caffeine in muscles of CD36 knockout mice (J. Lally and A. Bonen, unpublished data). These latter studies on CD36 knockout mice have also revealed that the reactive fatty acid esters (SSO, SSP) do not exert nonspecific inhibitory effects on other fatty acid transporters or on protein-independent fatty acid uptake.

The portion of CD36 that is stored in intracellular compartments is estimated to be $\sim 50 \%$ both in skeletal muscle (44) and heart (281). This intracellular CD36 depot was found to be enriched within subcellular fractions containing GLUT4 and the transferrin receptor, an endosomal protein (44). Hence, just like GLUT4, CD36 appears to recycle between endosomes and the sarcolemma. However, one report failed to observe an intracellular CD36 depot in human muscle (464). This observation appears to be anomalous, as it is not supported by another similar microscopic study in human muscle (236) or in the many subcellular fractionation studies in humans $(18,48)$ and animals $(71,73-76,164$, 219, 272, 273, 277, 278, 282).

To morphologically characterize CD36 translocation without using cell-disrupting procedures, Chinese hamster ovary $(\mathrm{CHO})$ cells stably expressing CD36 were created (460). Immunofluorescence microscopy revealed 


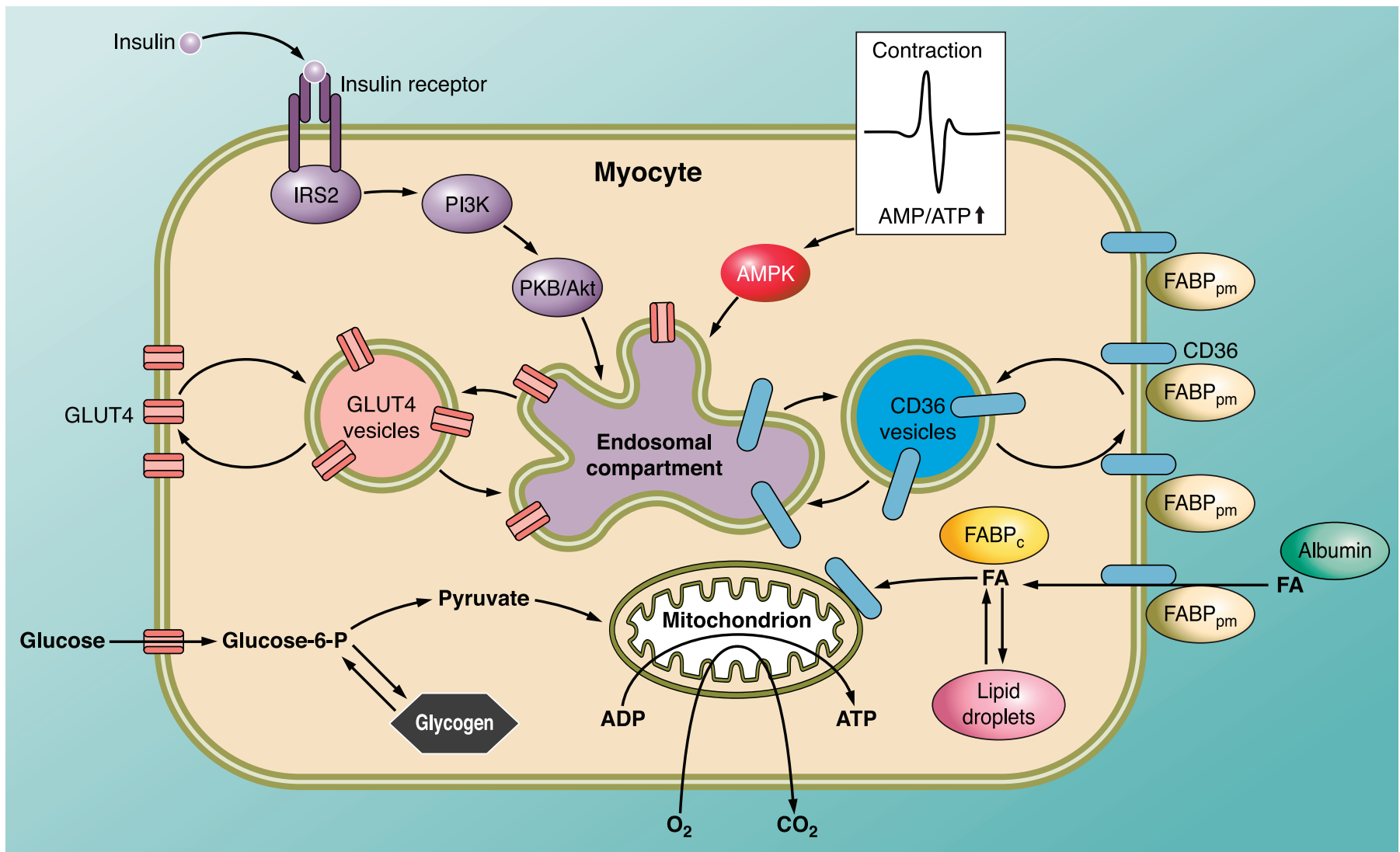

FIG. 4. Similarity between the regulation of cellular uptake of fatty acids and glucose. The uptake of both fatty acids and glucose by cardiac and skeletal muscle is increased after translocation of specific transporter proteins (shown for CD36 and GLUT4, respectively) to the sarcolemma in response to stimulation with insulin or during increased contractile activity. CD36 and GLUT4 may be mobilized from different stores within the endosomal compartment. Note that, for clarity, the involvement of GLUT1 in glucose uptake and the recycling of other fatty acid transporters $\left(\mathrm{FABP}_{\mathrm{pm}}\right.$ and FATPs) are not shown, while recently it has been observed that they can also be induced to translocate, at least in skeletal muscle (219). FA, (long-chain) fatty acid.

CD36 to be located both intracellularly and at the plasma membrane in a punctuate pattern. Upon treatment of these cells with various metabolic stimuli, the punctuate staining of CD36 at the plasma membrane increased by 1.7-fold, indicating the existence of specialized plasmalemmal regions involved in clustering of already present and newly translocated CD36 $(460,461)$. Whether these CD36 docking regions represent caveoli is uncertain, given the conflicting evidence on the role of caveolins in fatty acid transport (see sect. $\mathrm{III} D$ ).

Taken together, there is considerable evidence that CD36 is present within an endosomal compartment in muscle and heart. Whether GLUT4 and CD36 are present within the same (presumably) endosomal compartment, or are stored in different subcompartments within the endosomes, awaits further study. Unexpectedly, CD36 has also been located at the mitochondria where it appears to contribute to regulating fatty acid oxidation (see sect. $\mathrm{IV} C$ ). Because the mitochondria are not integrated with the recycling compartments encompassing the endosomes, it is very unlikely that mitochondria present a CD36 storage site for translocation to the cell surface. As yet, little is known about the dynamic distribution of other fatty acid transporters between intracellular stores and the plasma membrane.

\section{Endocrine-mediated and pharmacologically induced regulation of fatty acid transport and transporters}

It is well known that the uptake of glucose for cellular energy metabolism is regulated by the reversible translocation of the glucose transporter GLUT4 from endosomal compartments to the sarcolemma in muscle and heart, not only by changes in contraction, but also by insulin and selected pharmacological agents (oligomycin, AICAR, vanadate, arsenite) (24, 265, 267, 277, 282, 440). Recent work in our group and by others has shown that insulin and leptin, as well as the pharmacological agents oligomycin, AICAR, and dipyridamole, induce the translocation of CD36 in skeletal muscle $(164,278,305)$ and heart $(73,153,274,281,305,324)$. Insulin has also been reported to induce FATP1 translocation in adipocytes (399), but this insulin-induced FATP1 translocation in adipocytes was not observed in another study (346), or in the heart $(73,152)$. Insulin also failed to induce the trans- 
location of $\mathrm{FABP}_{\mathrm{pm}}$ in heart (73). However, in skeletal muscle, we (219) and others (479) have shown that insulin does induce the translocation of FATP1 $(219,479)$, as well as FABP $_{\mathrm{pm}}(164,219)$ and FATP4 (219), but not FATP6 (219). These studies indicate that the regulation of translocation of $\mathrm{FABP}_{\mathrm{pm}}$ and selected members of the FATP family, but not CD36, appears to be tissue specific.

The recent observation that insulin-stimulated CD36 translocation and fatty acid uptake are additive to contraction-stimulated CD36 translocation and fatty acid uptake $(219,278)$, just as has been observed for GLUT4 translocation and glucose transport (264), strongly suggests that there are insulin- and contraction-responsive intracellular subcompartments within the recycling endosomes dedicated to CD36 storage, as has previously been shown for skeletal muscle GLUT4 (264). In contrast, the insulin- and contraction-induced increases in plasmalemmal $\mathrm{FABP}_{\mathrm{pm}}$, FATP1, and FATP4 are not additive (219), suggesting that unlike CD36, there are no distinct insulinand contraction-responsive endosomal subcompartments for these transporters.

\section{B. Posttranslational Modification of Fatty Acid Transporters}

Subcellular translocation of fatty transporters has been shown to rapidly upregulate fatty acid uptake into (at least) heart and skeletal muscle (see sect. IvA). Additionally, other posttranslational mechanisms exist that could provide a further level of short-term regulation of fatty acid fluxes. These include palmitoylation, phosphorylation, and ubiquitination of fatty acid transporters. All three possible mechanisms are known to occur within a time scale compatible with short-term regulation.

\section{Palmitoylation of fatty acid transporters}

Palmitoylation and myristoylation are the two major types of covalent modification of proteins by fatty acids. Whereas protein myristoylation is a cotranslational process and a constitutive type of modification, protein palmitoylation is a short-term inducible event under hormonal regulation. Protein palmitoylation involves a thioester linkage catalyzed by specific protein fatty acyl-transferases and is rapidly reversed by deacylases (25). Moreover, protein palmitoylation is considered important in protein trafficking, especially in targeting proteins to caveolae (149). As mentioned in section $\mathrm{m} B$, CD36 possesses four palmitoylation sites within the two small intracellularly located $\mathrm{NH}_{2}$ - and COOH-terminal domains (427) (Fig. 1B).

Interestingly, insulin, one of the major physiological stimuli of fatty acid uptake, potently induces palmitoylation of CD36 in adipocytes (224). It has not yet been investigated whether CD36 palmitoylation also occurs in heart and muscle, and whether this palmitoylation can alter the transport activity of CD36. It is also possible that CD36 palmitoylation is not occurring independently of CD36 translocation, but is one of the regulating steps in insulin-induced CD36 translocation. The latter would be in nice agreement with the proposed function of protein palmitoylation, i.e., protein trafficking (149). Specifically, CD36 palmitoylation could target CD36 to caveolae, allowing the notion that these plasma membrane microdomains could serve as a surface docking station for CD36 (see sect. III $D$ ). Palmitoylation of the other fatty acid transporters, i.e., $\mathrm{FABP}_{\mathrm{pm}}$ and the FATPs, has not been reported.

\section{Phosphorylation of fatty acid transporters}

Protein phosphorylation is not only involved in rapid alterations in enzymatic activity of, for instance, protein kinases, but also in altering the intrinsic transport activity of membrane transporters, such as the L-type calcium channel (453). CD36 has been shown to possess at least two phosphorylation sites, one being a consensus protein kinase C (PKC) phosphorylation site at Thr-92 and one protein kinase A (PKA) phosphorylation site at Ser-237, which are both located within the extracellular loop (Fig. $1 B$ ). PKC-mediated CD36 phosphorylation has only been studied in platelets and is involved in determining the ligand specificity of CD36. In resting platelets, CD36 is constitutively phosphorylated and binds mainly to collagen. Platelet activation triggers the release of alkaline phosphatase, which leads to dephosphorylation of CD36, accompanied by a loss of collagen binding to CD36 and an increase in thrombospondin binding (13). However, the role of CD36-Thr-92 phosphorylation in fatty acid transport has not yet been examined. In contrast, the Ser-237 site has been associated with the regulation of the transport activity of CD36. In platelets, CD36-Ser-237 is phosphorylated by a cAMP-dependent ectokinase present at the surface of platelets (177) when these platelets are short-term incubated with cAMP and ATP. This CD36-Ser237 phosphorylation has been shown to modestly inhibit fatty acid uptake by human platelets (151). However, the functional significance of these findings is not yet clear because it is not known whether there are physiological conditions where cAMP and ATP are simultaneously present at the outer surface of the platelets. Moreover, whether ecto-PKA activity can regulate fatty acid uptake in cells other than platelets is also not known. Finally, to date, regulation of $\mathrm{FABP}_{\mathrm{pm}}$ and FATPs by phosphorylation has not been reported.

\section{Ubiquitination of fatty acid transporters}

Covalent linkage of proteins to ubiquitin, a 76-amino acid peptide, is a regulatory posttranslational modification enabling rapid degradation of these proteins. Lysine residues at proteins to be degraded are conjugated to the 
$\mathrm{COOH}$ terminus of ubiquitin by the subsequent action of ubiquitin-activating enzymes, ubiquitin-conjugating enzymes, and ubiquitin-protein ligases, after which the protein is targeted for degradation by the proteasome (83). CD36 contains two lysine residues (Lys-469 and Lys-472) within the small intracellular $\mathrm{COOH}$-terminal domain that appear to be major ubiquitination sites. CD36 ubiquitination is under hormonal and nutritional control because treatment of $\mathrm{C} 2 \mathrm{C} 12$ muscle cells for 30 min with insulin or fatty acids has been found to inhibit or stimulate ubiquitination of CD36, and thereby prevent or accelerate its degradation, respectively (388). These changes were paralleled by similar changes in fatty acid uptake. Combined with the effects of insulin on CD36 subcellular localization (see sect. $\mathrm{IV} A$ ), it appears that insulin treatment of myocytes increases sarcolemmal CD36 levels at two different posttranslational mechanisms, i.e., via induction of CD36 translocation to the sarcolemma, and via protection of sarcolemmal CD36 from degradation. Ubiquitination of $\mathrm{FABP}_{\mathrm{pm}}$ and FATPs has not yet been reported.

\section{Functioning of Fatty Acid Transporters in Mitochondrial Fatty Acid Utilization}

The idea that the rate of fatty acid oxidation by muscle tissues is dictated by the rate of delivery of fatty acids (concentration $\times$ blood flow) (155) is undergoing a reassessment. As outlined above, this reevaluation is based on recent experiments showing that fatty acid uptake is regulated at the plasma membrane by the presence of one or more fatty acid transporters. Interestingly, several fatty acid transporters are also present at the mitochondria where they may contribute to regulating fatty acid oxidation in concert with carnitine-palmitoyltransferase (CPT)-I.

\section{Fatty acid oxidation}

After entering muscle cells, a portion of the fatty acids are activated by acyl-CoAs to long-chain fatty acylCoAs in preparation for their import into mitochondria, where they are oxidized to provide ATP for many cellular processes. The CPT system is critically involved in the movement of these fatty acyl-CoAs across the mitochondrial membranes. CPT-I catalyzes the transesterification of fatty acyl-CoA to acyl-L-carnitine. The acyl-L-carnitine can then be translocated to the inner mitochondrial membrane by carnitine:acyl-L-carnitine translocase (CACT), and finally acyl-CoA is regenerated from acyl-L-carnitine by the latent CPT-II within the mitochondrial matrix (240). However, while CPT-I activity is allosterically inhibited by malonyl-CoA (for review, see Refs. 296, 400), the reduction in this malonyl-CoA, and changes in some other regulators, cannot fully account for the CPT-I-mediated increase in fatty acid oxidation that occurs during exercise $(31,317,318,356,402)$. Thus other processes must also be involved in upregulating fatty acid oxidation in muscle tissues during exercise.

Since it is known that some transport proteins, such as monocarboxylate transporters-1 and -2 (MCT-1 and -2) that transport lactate and pyruvate $(21,481)$, as well as mAspAT (69), a protein identical to $\mathrm{FABP}_{\mathrm{pm}}(26,69,217$, $419,485)$, are present at both the plasma membrane $(45$, $213,286)$ and the mitochondrion $(21,26,69,217,419,481$, $485)$, it has been suggested that fatty acid transport proteins could also be present in mitochondria, where they could possibly be involved in facilitating the movement of fatty acids into the mitochondria.

\section{Mitochondrial $F A B P_{p m}$}

As noted above, $\mathrm{FABP}_{\mathrm{pm}}$ and mAspAT are identical proteins (see sect. IIIA). Transfecting 3T3 fibroblasts (217) or rat skeletal muscle $(85,200)$ with mAspAT cDNA increased the content of plasmalemmal $\mathrm{FABP}_{\mathrm{pm}}$ and the rate of fatty acid transport into 3T3 fibroblasts (217) and skeletal muscle $(85,200)$. Concurrently, mitochondrial $\mathrm{FABP}_{\mathrm{pm}}$ was also increased. However, this failed to alter fatty acid oxidation in isolated mitochondria (200). Instead, mAspAT activity increased in proportion to its mitochondrial overexpression $(r=0.75)$ (200). Thus it appears that $\mathrm{FABP}_{\mathrm{pm}} / \mathrm{mAspAT}$ has two distinct functions depending on its subcellular location: 1) at the plasma membrane $\mathrm{FABP}_{\mathrm{pm}}$ contributes to fatty acid transport across the plasma membrane, but 2) at the mitochondrion mAspAT is involved in NADH transport across mitochondrial membranes (200).

\section{Mitochondrial CD36 and FATP1}

Studies in rat $(64,197,200,379,481)$ and human muscle $(30,198,200,203,372)$ have shown that CD36 (30, 64, 197, 198, 200, 203, 372, 481) and FATP1 (379) are present at both the plasma membrane and the mitochondrion. In addition, in skeletal muscle, CPT-I coimmunoprecipitated with CD36 (64, 372, 379) and FATP1 (379). Thus it appears that CD36 and CPT-I, and FATP1 and CPT-I, could be involved in regulating mitochondrial fatty acid oxidation. Evidence for this was reported in L6E9 myotubes in which overexpression of mitochondrial CD36 or FATP1 increased fatty acid oxidation (379).

Similar studies have yet to be performed in mammalian muscle. Nevertheless, strong support for a role of CD36 in skeletal muscle mitochondrial fatty acid oxidation has been shown in a number of studies. For example, there is a high correlation between the rate of palmitate oxidation in isolated mitochondria and the combined effects of mitochondrial CPT-I activity and CD36 content (i.e., multiple regression, $R=0.90$ ) (30). Further support came from studies in which rat muscles were induced to contract by electrical stimulation (30 $\mathrm{min})$ (64) or by 
exercise ( $2 \mathrm{~h}$ bicycle ergometry) (198). Mitochondria isolated from these muscles showed an increase in the content of CD36 $(64,198)$, which correlated with an increased rate of mitochondrial fatty acid oxidation $(r=0.63)$ in human muscle (198). These data suggest that CD36 translocates from an as yet unidentified CD36 storage compartment to the mitochondria. It remains to be determined whether the recycling endosomes, the intracellular storage pool for sarcolemmal CD36 (see sect. IVA), might also provide a storage compartment for mitochondrial CD36.

Further human studies showed that there was a good correlation between mitochondrial CD36 and mitochondrial fatty acid oxidation in lean and obese women $(r=$ 0.67), independent of body mass index (BMI) (203). Increasing skeletal muscle CD36 protein expression, either by chronic muscle stimulation in rats (7 days) (64) or with a program of weight loss and exercise training in humans (372), concomitantly increased fatty acid oxidation and mitochondrial CD36. Moreover, the increased rate of whole body fatty acid oxidation was correlated with an increase in the CD36 associated with CPT-I $(r=0.93)$, but not with CPT-I alone $(r=0.16)$ (372). Further support for the involvement of CD36 with regulating mitochondrial fatty acid oxidation came from studies with SSO, a specific blocker of plasmalemmal CD36 $(153,334)$ (see sect. IvA1). This CD36 inhibitor reduced fatty acid oxidation by $>80 \%$ in mitochondria obtained from both resting and exercised skeletal muscle $(30,64,198)$, although more recent work has found that SSO is not CD36 specific in mitochondria $(199,248)$. Because of this, the contribution of CD36 in the regulation of mitochondrial fatty acid oxidation has been questioned (248). In contrast, we (199) and others (379) do observe a role for CD36 in mitochondrial fatty acid oxidation. Specifically, 1) in CD36 null mice there is a reduced capacity for mitochondrial fatty acid oxidation in resting muscle and a sharply diminished ability to increase mitochondrial fatty acid oxidation in response to muscle contraction (199), 2) overexpressing mitochondrial CD36 in L6E9 myotubes increased the rates of fatty acid oxidation (379), and 3) mitochondrial fatty acid oxidation and mitochondrial CD36, but not CPT-I, are increased in muscle of obese Zucker rats (199). Taken together, there is strong evidence to suggest that CD36 and possibly FATP1 are involved, along with CPT-I, in regulating mitochondrial fatty acid oxidation.

It is questionable whether in mammalian muscle FATP1 is as effective as CD36 in regulating fatty acid metabolism. Presently, it is not known whether FATP1, like CD36, is induced to translocate to the mitochondrion, when muscle metabolic demand is increased. Moreover, recent work has shown that under basal conditions FATP1 has 1) a considerably lower fatty acid transport capacity and 2) a much lower stimulatory effect on fatty acid oxidation than CD36 in intact muscle (315). This may suggest that CD36 is likely more important than FATP1 in contributing to the regulation of mitochondrial fatty acid oxidation.

The mechanism(s) by which CD36 in combination with CPT-I contributes to the regulation of mitochondrial fatty acid oxidation is still unclear. We speculate that mitochondrial CD36 could perform a function that mirrors that of plasmalemmal CD36. Namely, upon cellular fatty acid uptake, plasmalemmal CD36 donates the fatty acids to $\mathrm{FABP}_{\mathrm{c}}$, the vehicle for cytoplasmic fatty acid transport to, among others, the mitochondria (142). This transfer of fatty acids most likely involves a direct interaction between CD36 and $\mathrm{FABP}_{\mathrm{c}}$ (396). Conversely, mitochondrial CD36 could facilitate the unloading of $\mathrm{FABP}_{\mathrm{c}}$ by accepting the fatty acids for transfer to ACS for activation.

Given that CD36 can be induced to translocate to both the plasma membrane and to the mitochondria, it appears that the rate of fatty acid oxidation is not solely dependent on the rate of fatty acid delivery (concentration $\times$ blood flow) to the muscle tissues, as has long been thought, particularly during exercise (155). We are now proposing an alternate model for upregulating skeletal muscle fatty acid oxidation, one that involves the muscle contraction-induced subcellular trafficking of CD36. Specifically, the proposed model (201) is a highly regulated and coordinated two-site system that responds rapidly to the muscles' metabolic needs to increase fatty acid oxidation during exercise. In this model muscle contraction or exercise 1) induces the translocation of CD36 to the plasma membrane, thereby increasing fatty acid uptake into the muscle cell (see sect. IvA), and 2) induces the translocation of CD36 to the mitochondrion, where it interacts with CPT-I to increase fatty acid uptake into the mitochondrial matrix to be oxidized (see above). This scheme does not imply that 1) other fatty acid transporters are not involved and 2) an increased delivery of fatty acids via the circulation is unimportant. Rather, this proposed model provides a means whereby intracellular signals initiated by muscle contraction can very rapidly (minutes) to regulate the increase in energy supply into the tissue towards the site of ATP synthesis so as to meet the increased metabolic demands.

\section{Coordinated Functioning of Fatty Acid Transporters}

It is conceivable that rapid fluctuations in metabolic needs of the heart and skeletal muscle require a distinct mechanism for fatty acid uptake, one that is different from the system that supports fatty acid entry, or release, from adipose cells. The possibility that selected fatty acid transporter proteins might function in a coordinated manner was suggested in the previous decade (142, 143, 286), and there is some experimental support for this idea. 
Studies in skeletal muscle and cardiac myocytes have shown that fatty acid uptake was blocked independently, by inhibitors of CD36 and $\mathrm{FABP}_{\mathrm{pm}}$, but their effects were not additive (286), suggesting that these two proteins interact with each other to take up fatty acids. Support for this has also been found in other studies. For example, the insulin- and AICAR-stimulated increases in fatty acid uptake did not correlate well with only plasmalemmal CD36 or with only plasmalemmal $\mathrm{FABP}_{\mathrm{pm}}$. Instead, the rate of fatty acid uptake was linearly related to the sum of plasmalemmal CD36 and plasmalemmal FABP ${ }_{\mathrm{pm}}$ (75). A similar observation occurred in heart and muscle of type 1 diabetic rats (272). Thus there is enticing evidence to suggest that $\mathrm{FABP}_{\mathrm{pm}}$ and $\mathrm{CD} 36$ may interact with each other. In addition, CD36 and caveolin-1 might interact with each other (see sect. IIID). Whether any of these latter proteins also interacts with FATPs is unknown, although this may occur as CD36 and FATP6 colocalize in the heart (139). As noted in section $\mathrm{Iv} B$, a concerted action among membrane fatty acid transporters, and other proteins such as cytoplasmic FABP $(143,396)$ and ACS-1 $(279,346)$, may allow a fine-tuning of fatty acid transport so as to have this substrate readily available for intracellular utilization.

\section{E. Do Fatty Acid Transporters Channel Fatty Acids to a Particular Metabolic Fate?}

It has been suggested, based on the overexpression of fatty acid transporters in different cell lines, that specific transport proteins interact with specific intracellular proteins to channel fatty acids to different metabolic fates within the cell $(20,136,176,346,487)$. Some evidence was recently shown for this in 3T3-L1 adipocytes, in which FATP1 and ACS1 coimmunoprecipitate (346). Overexpression of ACS1 increased the rate of fatty acid transport, leading to the conclusion that constitutive interaction between FATP1 and ACS1 contributes to the efficient cellular uptake of fatty acids in adipocytes through vectorial acylation. This led to the suggestion that fatty acid esterification might be required to stimulate fatty acid uptake into adipocytes (346). Others have shown a role for ACS1, -4 , and -6 in contributing to fatty acid transport (432). However, as reviewed above, fatty acid transport is not dependent on activation by ACS1, but, in vivo, these two processes (fatty acid transport and activation) are likely complementary (see sect. IIIC).

While fatty acid channeling via different fatty acid transporters to different intracellular fates is an attractive hypothesis to account for the large number of fatty acid transporters expressed in a number of tissues, supporting evidence is only available from studies in vitro, in selected cell lines in which normal physiological regulation is largely absent. Moreover, unlike skeletal muscle and the heart, very rapid adjustments in fatty acid provision are not required in quiescent cells with a low metabolic rate.

In vivo, fatty acid channeling to a particular metabolic fate via specific fatty acid transporters would not seem to hold, particularly in metabolically dynamic tissues such as heart and skeletal muscle. Such a scheme ignores the important role of physiological signals such as hormones (insulin) and muscle contraction. Each of these stimuli can induce the translocation of $\mathrm{FABP}_{\mathrm{pm}}$, CD36, FATP1, and FATP4 (219), thereby increasing the rate of fatty acid uptake. Following their uptake, the fate of these transported fatty acids is most likely regulated by insulinspecific and contraction-specific intracellular signals that direct fatty acids to esterification or oxidation, respectively, consistent with the metabolic needs of the tissue. Specifically, the contraction-induced increases in the sarcolemmal pool of CD36 (see sect. Iv $A$ ) are linked with enhanced fatty acid oxidation (44, 213, 277, 290, 305), and the insulin-induced increases in sarcolemmal CD36 with enhanced fatty acid esterification $(278,281)$. Observations in CD36 knockout animals further underscore this notion, as in AICAR-stimulated muscles CD36 ablation selectively impairs fatty acid oxidation while in insulin-stimulated muscles CD36 ablation results in a selective reduction of fatty acid esterification (42). In line with this, pharmacological blockade of CD36 in cardiomyocytes (287) or ablation of CD36 in muscle (42) in the absence of metabolic stimuli reduces basal rates of both muscle fatty acid uptake, esterification, and oxidation to the same extent, indicating that sarcolemmal CD36 does not channel fatty acids specifically into oxidation or esterification. Together, these observations strongly indicate that the metabolic machinery rather than sarcolemmal CD36 per se determines the ultimate intracellular metabolic fate of incoming fatty acids.

The metabolic fate of fatty acids that have been transported into heart and muscle is dictated by intracellular signals, fashioned by the energetic demands, i.e., contractile activity $(112,277,385,386)$ and/or the endocrine milieu (e.g., insulin and leptin; Refs. 115, 278, 281, 309, 310, 405). For contractile activity and leptin, the intracellular signal is AMP-kinase activation, which exerts an inhibitory phosphorylation of acetyl-CoA carboxylase, thereby lowering the intracellular malonyl-CoA concentration. Consequently, CPT-I becomes deinhibited, allowing the mitochondrial $\beta$-oxidation rate to increase (270, $400)$. With respect to insulin, this anabolic hormone has been found, at least in adipocytes, to acutely upregulate the activity of glycerol-3-phosphate acyltransferase (GPAT; the rate-limiting enzyme in triacylglycerol synthesis) (462), likely through phosphorylation within a consensus motif for casein kinase-2 (56), a known protein kinase target of insulin signaling (247). Whether recruitment of other fatty acid transporters and/or members of the ACS family respond differentially to these stimuli to act in concert with CD36 
possibly to channel fatty acids to different fates is not known.

In summary, translocation of fatty acid transporters provides a very rapid, localized regulation of lipid metabolism at the level of the plasma membrane. In contracting muscles we propose that this may be accomplished by coordinating fatty acid uptake and mitochondrial oxidation via translocation of CD36 to these subcellular locations at the same time. Cofunctioning of fatty acid transporters at the plasma membrane has been shown and may also occur at other subcellular locations, such as mitochondria. Additional levels of regulation may exist, including possibly ectophosphorylation of CD36 to diminish fatty acid uptake, as has been shown in human platelets (151), CD36 palmitoylation (427), and association with specific membrane microdomains such as lipid rafts (117). Channeling of fatty acids to a particular metabolic fate within the muscle cells, in vivo, is likely directed by intracellular metabolic signals generated by muscle contraction or the endocrine milieu, and may involve selective recruitment of one or more fatty acid transporters and/or members of the ACS family depending on the physiological signal (e.g., contraction or hormones).

\section{SIGNALING AND TRAFFICKING EVENTS REGULATING MEMBRANE TRANSPORTER TRANSLOCATION}

In the previous section it was outlined that fatty acid uptake is regulated by a translocation of fatty acid transporters from intracellular stores to the sarcolemma. Hence, the sarcolemmal presence of these transporters is not fixed, but can be altered dynamically in coordination with the continuously changing metabolic demands of heart and muscle.

CD36 and $\mathrm{FABP}_{\mathrm{pm}}$ are examples of proteins, with cell surface-related functions (receptors and transporters), that recycle to and from the sarcolemma. Even under basal, nonstimulated conditions (low metabolic demand) and consequently a low fatty acid uptake rate, there is no static distribution of fatty acid transporters, but instead a continuous recycling between the endosomal compartment and the sarcolemma in such a manner that the combined rates of endocytosis and exocytosis result in a low basal surface localization (for review, see Ref. 276). Theoretically, increased exocytosis or decreased endocytosis could result in net transporter relocation. From extensive research on GLUT4 translocation in adipocytes, it would seem that a net transporter redistribution to the sarcolemma upon exposure of cardiac tissue or cells to a given stimulus is caused by an increase in the rate of exocytosis without any change in the rate of endocytosis (which is regarded as noninducible in this translocation model). This is currently the most favored view of the regulation of transporter translocation (207, 365,
428), although a decreased endocytosis may also contribute to net GLUT4 translocation (263). Nevertheless, in general, endocytosis is regarded as an unregulatable (housekeeping) trafficking event, while in contrast exocytosis is highly inducible.

Conceptually, the events regulating the net translocation of a given recycling protein, i.e., CD36, can be subdivided into 1) stimulus-induced signaling and 2) induction of trafficking processes, presumably acting on acceleration of exocytosis (Fig. 5). The signaling events can be induced by various mechanical, hormonal, or pharmacological stimuli (see sect. IvA). Each of these stimuli will induce the activation of key/master protein kinases, which initiate a pleiotrophy of signaling cascades, allowing cells to perform the full range of responses needed to adequately respond to changing metabolic conditions. With respect to regulation of subcellular transporter distribution, one (or more) of these stimulus-induced signaling cascades will activate the vesicular trafficking machinery, thereby modulating the continuous recycling of membrane receptors and transporters to achieve a net translocation of these proteins.

\section{A. Signaling Pathways}

Insulin and contraction are the main physiological stimuli able to induce fatty acid uptake through translocation of CD36 and/or FABP ${ }_{\mathrm{pm}}$, as well as FATP1 and -4 . Both stimuli activate distinct signaling cascades. Whereas the insulin-signaling cascade starts from the extracellular leaflet of the sarcolemma, the origin of the contraction signaling cascade is intracellular.

\section{Insulin signaling}

The components involved in the postreceptor insulin signaling pathway, that induce GLUT4 translocation to stimulate glucose transport, are well known and have been the topic of extensive research during the last two decades. This signaling pathway may also be involved in insulin-stimulated fatty acid transporter translocation. Therefore, we briefly review below the sequence of signaling events involved in insulin-stimulated GLUT4 translocation, as a framework for examining insulin-stimulated fatty acid transporter (particularly CD36) translocation.

A) INSULIN SIGNALING PATHWAYS IN GLUT4 TRANSLOCATION. The binding of insulin to the $\alpha$-subunit of the insulin receptor induces a conformational change throughout the whole protein, resulting in the autophosphorylation of the receptor $\beta$-subunit at tyrosine residues, thereby providing docking sites for phosphotyrosine-binding (PTB) domain containing proteins, most critically the insulin receptor substrates (IRS), of which IRS-1 is the key player in muscle and the heart. In addition, members of the class 1 phosphatidylinositol-3 kinases (PI3Ks) are activated by 

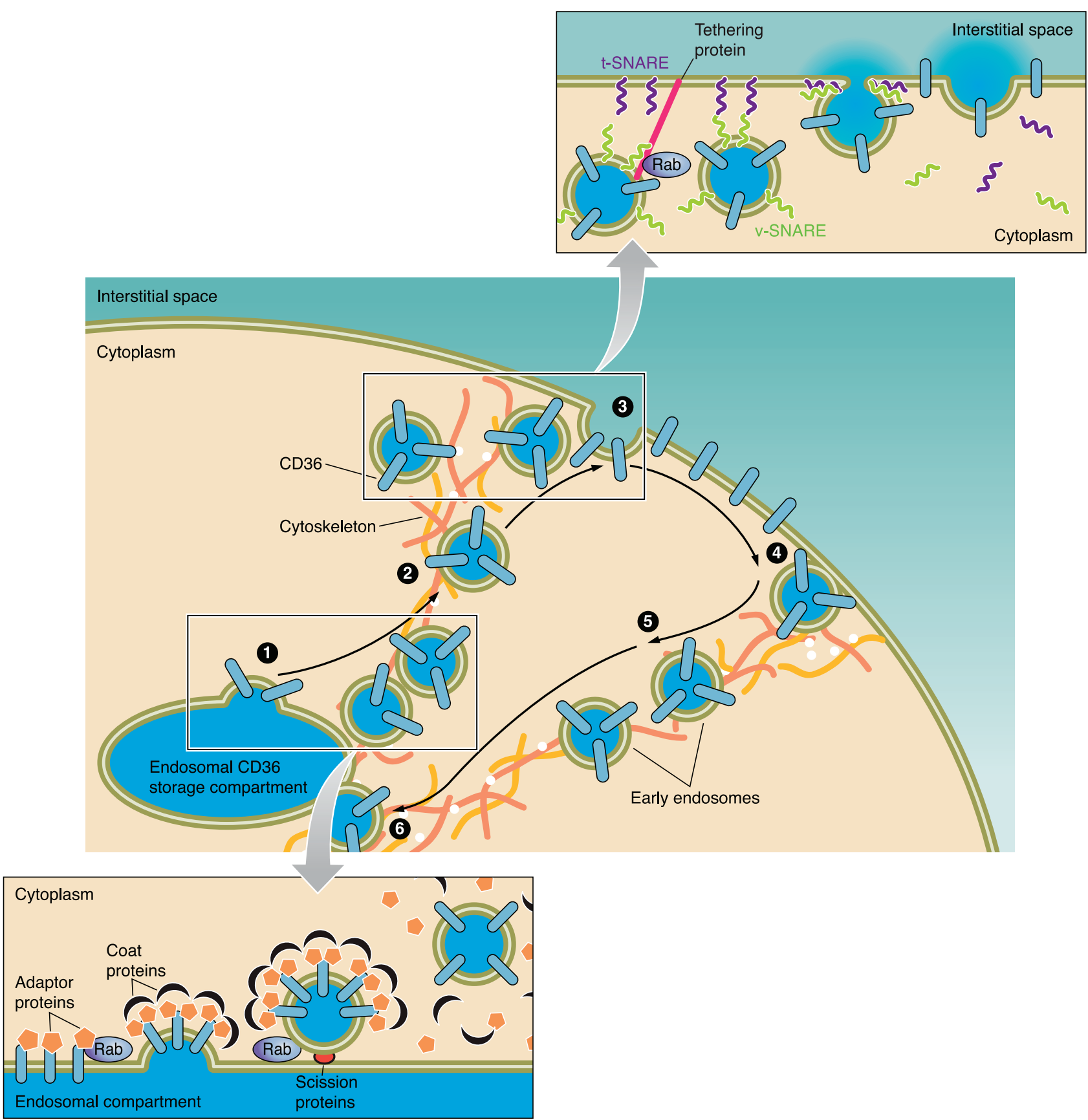

FIG. 5. Trafficking pathways involved in CD36 recycling. The transition of CD36 from the endosomal storage compartment to the plasma membrane, and vice versa, is regulated by several protein families with different functions. 1, Bottom inset: adaptor proteins and Rab-GTPases regulate the binding of coat proteins to areas enriched in CD36. The formation of coat complexes leads to the generation of a CD36 transport vesicle which is released to the cytoplasm by scission proteins. After budding of the vesicle, the adaptor and coat proteins again detach. 2: Upon binding of a motor protein, the transport vesicle is moved along cytoskeleton filaments to its designated target membrane. Also this process is initiated by Rab-GTPases. 3, Top inset: tethering proteins trap the transport vesicle below the cell surface. The interaction of a specific subset of vesicleassociated SNAREs (v-SNAREs) and target-membrane associated SNAREs (t-SNAREs) brings the vesicle to such a close proximity to the plasma membrane that the water barrier can be overridden and fusion of the two membranes can occur. After fusion, the SNARE complex disintegrates. 4-6: The transport of CD36 from the plasma membrane to the endosomal compartment proceeds analogous to steps 1-3.

the binding of its p85 regulatory subunit to the phosphorylated IRS-1, thereby recruiting PI3K to the plasma membrane. PI3Ks form a family of enzymes phosphorylating the $D-3$ position on the inositol ring in phosphoinositides, and class 1 PI3Ks specifically convert phosphatidylinositol 4,5-bisphosphate into phosphatidylinositol 3,4,5trisphosphate $\left(\mathrm{PIP}_{3}\right)$ for downstream activation of insulin signaling. Investigations on the involvement of 
PI3K in GLUT4 translocation have been greatly facilitated by the use of pharmacological inhibitors, such as wortmannin and LY294002. Numerous publications report that both inhibitors largely abolish insulin-induced GLUT4 translocation and glucose uptake (for review, see Ref. 381).

The lipid signaling intermediate $\mathrm{PIP}_{3}$ is not only regulated by its PI3K-mediated formation, but also by its degradation rate by inositol phosphatases, namely, $\mathrm{SH} 2$ domain-containing 5 '-inositol phosphatase (SHIP)-2, and the tumor suppressor gene product PTEN (phosphatase and tensin homolog deleted on chromosome 10), dephosphorylating $\mathrm{PIP}_{3}$ at the $3^{\prime}$ position. Hence, these inositol phosphatases are involved in the regulation of GLUT4 translocation $(368,426)$. The formation of $\mathrm{PIP}_{3}$ at the plasma membrane directly drives the activation of a number of different protein kinases with lipid binding domains, of which three have now been recognized as essential players in insulin-induced glucose uptake, namely, Akt/protein kinase B (PKB), PKC- $\lambda / \zeta$, and 3-phosphoinositide-dependent protein kinase-1 (PDK1). Binding of $\mathrm{PIP}_{3}$ induces a conformational change within these kinases resulting in their autophosphorylation or plasma membrane binding.

The importance of Akt, especially isoform 2, in the regulation of glucose uptake has been revealed in Akt2 null mice $(81,137)$. The activation of Akt is complex in that it requires dual phosphorylation at Ser-473 and Thr309 in addition to its PI3K-mediated recruitment to the plasma membrane. The Thr-309 phosphorylation within the activation loop is mediated by PDK1 (9), in which involvement in cardiac insulin-stimulated glucose uptake was subsequently demonstrated by the inability of cardiac myocytes from muscle-specific PDK1 null mice to increase glucose uptake upon insulin treatment (306).

In parallel to Akt2, PKC- $\zeta$ is activated by PDK1, upon unfolding of the pseudosubstrate domain and exposure of the activation loop Thr-410 site (122). The involvement of PKC- $\lambda / \zeta$ in insulin-induced GLUT4 translocation and glucose uptake has been convincingly proven in muscle cell lines expressing dominant-negative mutants (17). The simultaneous and combined activation of Akt 2 and PKC- $\lambda / \zeta$ is necessary for insulin-induced GLUT4 translocation in both heart (29) and skeletal muscle (122). Akt is known to activate a great number of substrates involved in a pleiotrophy of anabolic responses. Among these, the phosphorylation of an Akt substrate of $160 \mathrm{kDa}$ (AS160) has been found to be particularly important for GLUT4 translocation $(367,483)$. Insulin-stimulated phosphorylation of AS160 (IR $\rightarrow$ IRS1-PI3K $\rightarrow$ Akt2 $\rightarrow$ AS160) releases the inhibition on vesicular GLUT4 movement to the plasma membrane, as phosphorylated AS160 can no longer maintain rab proteins in a GDP-bound state. Although PKC- $\lambda / \zeta$ targets involved in insulin-induced GLUT4 translocation have not been examined intensively, it appears that PKC- $\lambda / \zeta$ binds to intracellular GLUT4 compartments upon insulin stimulation and directly phosphorylates the GLUT4 compartment-associated vesicle-associated membrane protein-2 (VAMP2) (52). Hence, Akt2-mediated AS160 phosphorylation, as well as PKC- $\lambda / \zeta$-mediated VAMP2 phosphorylation, are at the threshold between insulininduced signaling and trafficking events in the translocation of GLUT4 to the cell surface. Excellent, detailed reviews discussing more fully the insulin-stimulated signaling pathway involved in promoting GLUT4 translocation are available $(29,68)$.

B) INSULIN SIGNALING PATHWAYS IN FATTY ACID TRANSPORTER TRANSLOCATION. Similarly to insulin-induced GLUT4 translocation, the insulin-induced translocation of fatty acid transporters requires the activation of PI3K. Namely, inhibition of this kinase by either wortmannin or LY294002 inhibited fatty acid uptake and CD36 translocation in cardiac myocytes (281) and skeletal muscle (278). However, it cannot yet be deduced which class of PI3K contributes to insulinstimulated fatty acid uptake because wortmannin and LY294002 potently inhibit most isoforms (381). Likely, class II PI3Ks can be excluded because isoforms within this class are relatively resistant towards both inhibitors (108). Recent studies in L6 muscle cells and in primary cardiac myocytes using pharmacological inhibitors have found that downstream of PI3K, atypical PKC- $\zeta$, but not $\mathrm{PKB} /$ Akt, mediates the effects of insulin on fatty acid uptake (239) via translocation of CD36 (284). In contrast to L6 muscle cells, it does appear, however, that Akt-2 is required in mammalian muscle for insulin-induced fatty acid transport and for the translocation of selected fatty acid transporters. Specifically, we have found that insulin 1) failed to stimulate fatty acid and glucose transport in skeletal muscle of Akt-2 null mice, and 2) failed to induce the translocation of CD36 and FATP1, as well as GLUT4, while the translocation of $\mathrm{FABP}_{\mathrm{pm}}$ and FATP4 was not impaired (218). These observations indicate that insulinstimulated GLUT4 translocation and the insulin-stimulated translocation of some fatty acid transporters (CD36 and FATP1) are similarly regulated via the PI3K-Akt2 signaling pathway. It is possible, however, via the use of arsenite, to divorce the translocation of GLUT4 from that of CD36 (282). Similarly, CD36 translocation could be divorced from the translocation of $\mathrm{FABP}_{\mathrm{pm}}$ and of FATP4 in Akt-2 null mice (218). These observations indicate that insulin signaling pathways for GLUT4 and selected fatty acid transporters can at times diverge, either because signals diverge at some point beyond Akt-2 or different subcellular depots of fatty acid transporters are targeted.

\section{Contraction signaling}

The metabolic demands of muscle contraction favor the activation of nutrient and stress sensing cascades. Hence, an increase in contractile activity can result in a 
rapid rise in the concentration of a number of second messengers in heart and muscle, such as AMP, cAMP, $\mathrm{Ca}^{2+}$, and reactive oxygen species (ROS). Together these second messengers activate a complex network of signaling events (353). Among all the protein kinases activated by contraction [e.g., PKA, PKC- $\delta$ and $-\varepsilon$, extracellularly regulated protein kinases (ERK)-1 and -2 , mitogen-activated protein kinase (MAPK), and $\mathrm{Ca}^{2+} /$ calmodulin-dependent protein kinases (CaMK)], the activation of AMPactivated protein kinase (AMPK) is known to have a variety of metabolic actions $(275,352)$, including the stimulation of fatty acid oxidation via the phosphorylation and inactivation of acyl-CoA carboxylase (ACC) and the consequent reduction in malonyl-CoA which releases the inhibitory effects on CPT-I $(270,400)$. In line with this, we have shown that AMPK plays a crucial role in the translocation of CD36 $(73,277)$, and that of $\mathrm{FABP}_{\mathrm{pm}}$ (73). Importantly, some of the other mentioned contractionactivated kinases are not involved in contraction-induced fatty acid transport, including cAMP-activated PKA (289) and conventional or novel PKCs (288).

Based on the central role of AMPK in contractioninduced fatty acid transport, we will discuss which additional protein kinases could be involved in the AMPK signaling pathway to induce fatty acid transporter translocation. This will necessarily be confined to CD36, as similar details are currently not available for other fatty acid transporters.

A) INVOLVEMENT OF AMPK IN CONTRACTION-INDUCED FATTY ACID UPTAKE. AMPK is a heterotrimeric protein consisting of catalytic $(\alpha 1, \alpha 2)$ and regulatory $(\beta 1, \beta 2, \gamma 1, \gamma 2 \mathrm{a}, \gamma 2 \mathrm{~b}, \gamma 3)$ subunits $(168,170)$. Both AMPK $\alpha 1$ and AMPK $\alpha 2$ are expressed in heart and skeletal muscle, of which AMPK $\alpha 2$ is the predominant subunit (401). Activation of the AMPK complex occurs mainly via AMP by three independent mechanisms: 1) promotion of phosphorylation of Thr-172 within the $\alpha$-subunit by upstream kinases, 2) allosteric activation of the phosphorylated enzyme, and 3) inhibition of dephosphorylation of Thr-172 by protein phosphatases (171). Under conditions that favor a rapid increase in intracellular AMP, such as during increased contractile activity in muscle (477) or during a period of cardiac ischemia (259), AMPK becomes activated, resulting in the activation of catabolic pathways, such as the increased oxidation of fatty acids (exercise) and glucose (ischemia), and the deactivation of anabolic pathways, such as storage of these substrates (169).

Evidence that AMPK is involved in contraction-induced fatty acid transport comes from the observation that contraction-induced fatty acid uptake is not additive to that of agents able to activate AMPK and increase fatty acid transport. However, it must be recognized that some recent studies have begun to suggest that AMPK is not necessarily essential for the regulation of fatty acid oxidation by AICAR or muscle contraction $(116,357)$. Nevertheless, AMPK-acti- vating agents, oligomycin (a mitochondrial $\mathrm{F}_{1} \mathrm{~F}_{0}$-ATPase inhibitor) and AICAR (a cell-permeable adenosine analog, which is converted intracellularly to ZMP, an AMP analog), induce the translocation of CD36 $(73,277)$ and $\mathrm{FABP}_{\mathrm{pm}}(73)$ to the sarcolemma. Because muscle contraction (44) and these contraction-mimetic agents failed to stimulate fatty acid transport, either into skeletal muscle or cardiac myocytes (277) treated with the specific CD36 inhibitor SSO (44, 277), or in cardiac myocytes from CD36 null mice (153), it is evident that AMPK-activated induction of CD36 translocation is largely, if not entirely, responsible for increasing fatty acid transport. More direct evidence for the involvement of AMPK in contraction-induced fatty acid transport was obtained with cardiac myocytes from mice overexpressing an AMPK $\alpha 2$ kinase-dead subunit, since this dominant-negative mutant resulted in a severely reduced activation of both AMPK $\alpha 1$ and AMPK $\alpha 2$, and in a complete abolishment of oligomycin- or AICAR-induced fatty acid uptake (152). Similarly, oligomycin-induced fatty acid uptake was also completely lost in cardiac myocytes from AMPK $\alpha 2$ knockout mice, indicating that in the heart AMPK $\alpha 2$, not AMPK $\alpha 1$, is responsible for contraction-induced fatty acid uptake (152). In this respect, contraction-induced cardiac fatty acid uptake closely resembled contraction-induced GLUT4 translocation, which was also found to be entirely and selectively dependent on AMPK $\alpha 2$ activation in the heart (152).

Whether the translocation of CD36 and possibly other fatty acid transporters in skeletal muscle is also fully AMPK $\alpha 2$ dependent is questionable. It is now known that during muscle contraction the two $\alpha$-isoforms (AMPK $\alpha 1$ and AMPK $\alpha 2$ ) can substitute for each other in terms of activity, which may explain the normal glucose uptake despite the lack of either AMPK $\alpha 1$ or AMPK $\alpha 2$ in genetically altered mice (227). Thus the roles of AMPK $\alpha 1$ or AMPK $\alpha 2$ in promoting fatty acid transporter translocation and fatty acid uptake in skeletal muscle remain to be determined.

B) CONTRACTION SIGNALING UPSTREAM OF AMPK. In the late 1980s, kinase activity towards AMPK-Thr-172 (referred to as AMPKK) was detected as a distinct entity in a partial purification of AMPK from rat liver (67). However, it proved to be difficult in subsequent years to purify the kinase-kinase to homogeneity. Fortunately, independent research in yeast provided important clues in the identification of AMPKK. Namely, Snf1, the yeast homolog of AMPK, is phosphorylated by three kinases (204), and database searches revealed three closely related mammalian kinases, LKB1, CaMKK $\alpha$, and CaMKK $\beta$ (166). LKB1 has a ubiquitous expression pattern in mammalian tissues, whereas the expression of CaMKKs is restricted to brain, and to a lesser extent to muscle tissues (11; and see reviews in Refs. 167, 463). In vivo, LKB1 and CaMKK are each regulated differently. In muscle tissue, CaMKK $\beta$ activity is inducible, i.e., by an increase in intracellular $\mathrm{Ca}^{2+}$ (179). In contrast, LKB1 is constitutively active (363), but 
it can only activate AMPK during conditions when intracellular AMP is increased (e.g., exercise), as AMP binding to AMPK induces a conformational change within the AMPK complex so that Thr-172 becomes accessible to phosphorylation by LKB1 (178). While LKB1 has been shown to be involved in contraction-induced glucose uptake in muscle (364), it is still controversial whether CaMKK $\beta$ regulates contraction-stimulated glucose uptake in muscle via AMPK activation $(222,223,478)$.

Recently, cardiac myocytes from LKB1 null mice were used to examine oligomycin- and AICAR-stimulated fatty acid uptake (152). These studies demonstrated that the stimulatory effects of each of these AMPK-activating agents on fatty acid uptake and CD36 translocation were lost, indicating that LKB1 is the primary AMPKK involved in this metabolic action. Since AICAR and oligomycin each stimulates fatty acid uptake in a nonadditive manner to contraction $(152,277)$, the AICAR and oligomycin findings can be extrapolated to contraction, namely, that an LKB1-AMPK signaling axis is necessary for contractioninduced fatty acid uptake via CD36 translocation. In skeletal muscle, the regulation of contraction-induced fatty acid uptake may occur in part via $\mathrm{Ca}^{2+}$-dependent activation of CaMKK and AMPK $(343,344)$, but whether these signals induced the translocation of one or more fatty acid transporters was not determined.

C) CONTRACTION Signaling DOWNSTREAM OF AMPK. To date, close to 20 direct cellular targets of AMPK have been identified (434). This number is expected to grow given the critical role of AMPK in cellular energy homeostasis. A consensus recognition motif has now been identified around the site phosphorylated by AMPK (434). Proteins with these motifs include selected transcription factors and a few signaling proteins, as well as proteins involved in fatty acid and glucose metabolism. One signaling protein in particular stands out, namely, AS160. It is strongly implicated in insulin-induced GLUT4 translocation and is likely also involved in insulin-induced fatty acid transporter translocation, given that its upstream kinase Akt-2 is required for insulin-stimulated fatty acid transport and fatty acid transporter translocation (CD36 and FATP4; Ref. 218; see sect. vA1). Indeed, it is now firmly established that in skeletal muscle AS160 is phosphorylated in vivo both by insulin and by exercise, and in situ by electrically induced muscle contraction as well as by AICAR (68, 257), while AS160 phosphorylation is absent in mice deficient in AMPK signaling (435). It now appears that AS160 is a possible point of convergence for insulinand contraction-signaling pathways from where trafficking processes are activated for simultaneous GLUT4 and CD36 translocation.

There are also several protein kinases, most notably ERK-1/-2 and PKC- $\zeta$, reported to operate in contractionstimulated glucose uptake in skeletal muscle (79). Interestingly, ERK has also been proposed to be involved in contraction-induced CD36 and fatty acid uptake in skeletal muscle, based on the ability of the ERK inhibitor PD98059 to inhibit these events $(345,443)$. More recent work indicated that contraction-induced fatty acid uptake may occur in part via $\mathrm{Ca}^{2+}$-independent activation of ERK1/2 (343). Another protein kinase recently identified to be necessary for contraction-induced GLUT4 translocation is protein kinase D-1 (PKD1), which is activated independently from AMPK (288). Whether PKD1 has a role in contraction-induced fatty acid transporter translocation has not yet been assessed.

\section{B. Trafficking Pathways}

A sequence of trafficking events resulting in translocation of transporter proteins is initiated once the insulinor contraction-induced signaling cascades have been completed. The cellular machinery responsible for migration of cargo from one compartment to the other, and vice versa, is referred to as the trafficking machinery. Regulation of this transport needs to be unidirectional and extremely specific to ensure that vesicles containing cargo (e.g., CD36 and/or GLUT4) destined for the plasma membrane do not fuse randomly with membranes from other organelles (99). To provide the unidirectionality of intracellular transport, and the proper sorting mechanisms, each mammalian cell type contains a specific set of trafficking proteins, dedicated to adequately meet this specific function. Derived from research with nerve cells for the secretion of neurotransmitters and adipocytes for the translocation of GLUT4 (99), the list of proteins that make up the cellular trafficking machinery includes the following (Fig. 5).

\section{SNARE proteins}

Soluble $N$-ethylmaleimide attachment protein receptor (SNARE) proteins are responsible for safeguarding the specificity of the transport of intracellular membrane vesicles according to the principles of the so-called SNARE hypothesis (99). This hypothesis postulates that in vesicular trafficking events a unique vesicle-associated protein (v-SNARE) specifically recognizes and interacts with a cognate t-SNARE protein localized at the target membrane. This specific interaction results in the formation of a SNARE-pin complex necessary for the fusion of vesicles with their target compartments.

\section{Rab proteins}

Ras-related Rab GTP-binding proteins bind and hydrolyze GTP. In their cycling between GTP-bound (active) and GDP-bound (inactive) conformations, these proteins act as molecular switches in vesicular trafficking (102). Although Rabs have not been detected in the SNARE 
complex, they are probably required for the assembly of this complex, as has been shown in yeast (390). Members of the Rab family are associated with distinct intracellular compartments involved in endocytic and exocytic processes, indicating that the individual Rab proteins have specialized transport functions.

\section{Coat/scaffolding proteins}

Coat proteins are required for the generation of highly curved membranes and the assembly of accessory proteins into a "vesicle fission complex." Subcellular trafficking vesicles possess characteristic proteinaceous coat structures allowing their classification accordingly into 1) clathrincoated vesicles, 2) COP-coated vesicles, and 3) caveolincoated vesicles (caveolae). It is disputed whether these different classes indeed perform different trafficking actions (297).

\section{Other proteins}

Other proteins involved include motor proteins (dynamins), adaptor proteins for complex formation and cargo assembly ( $\beta$-arrestin), phosphatidylinositol kinases for generation of bilayer destabilizing phospholipids necessary for vesicle budding, GTPases involved in vesicle fission (ARFs), uncoating proteins (auxillin), filamentous proteins providing the "highway" along which the substrate transporter-containing vesicle travels to the cell surface (297).

Below we summarize the as yet limited information currently available on the involvement of some of these proteins in the regulation of fatty acid transporter recycling. These findings will be discussed against the background of a steadily growing list of proteins found to be involved in insulin-stimulated GLUT4 translocation.

A) SNARE PROTEINS. In muscle tissues, insulin-induced GLUT4 translocation is the most intensively studied process in relation to SNARE proteins. In these tissues, vesicleassociated membrane protein-2 (VAMP-2) appeared to be the v-SNARE involved, and syntaxin-4 the cognate t-SNARE, which operates in conjunction with the SNARE-related protein SNAP-23 (for review, see Refs. 127, 397). SiRNA experiments have indicated that GLUT4 exocytosis induced by hyperosmolarity (a PI3Kindependent stimulus) is not mediated by VAMP-2, but by VAMP-7, and that VAMP-8 is responsible for GLUT4 endocytosis (475). It is not yet known whether these same SNARE proteins are involved in fatty acid transporter translocation, and whether insulin-induced CD36 translocation requires identical or different SNAREs compared with contraction-induced translocation of CD36 or FABP ${ }_{\mathrm{pm}}$. However, the involvement of PKC- $\zeta$ in insulin-stimulated fatty acid uptake (possibly through CD36 translocation) might suggest that VAMP-2 is involved in insulin-induced CD36 transloca- tion similar to its involvement in insulin-induced GLUT4 translocation. Namely, VAMP-2 is recognized as a direct phosphorylation target of PKC- $\zeta$ (see sect. $\mathrm{vA1}$ ).

B) RAB PROTEINs. In muscle cells and adipocytes, at least three species of Rab proteins were found to be involved in the regulation of GLUT4 translocation: Rab 4 and Rab11 in insulin-stimulated GLUT4 exocytosis (109, 449), and Rab 5 in GLUT4 endocytosis (206). Accordingly, Rab5 has been localized to the cell surface $(60,439)$. Interestingly, Rab4 and Rab11 seem to be differentially localized: Rab4 is predominantly localized in a specialized insulin-responsive preendosomal compartment uniquely dedicated to the storage of GLUT4 (452), whereas Rab11 specifically associates with recycling endosomes in mammalian cell lines $(392,450)$. Both intracellular compartments are known to participate in trafficking of GLUT4 in muscle (8).

Only Rab11 has been found to participate in the regulation of trafficking of fatty acid transporters. First, Rab11 was found to be present in intracellular CD36 vesicles (308). Second, silencing of Rab11 in H9c2 cells increased basal cell surface content of CD36 (and GLUT4) (377). Since silencing of Rab11 did not alter the stimulus-induced translocation of CD36, it appears that Rab11 has a role in endocytotic rather than in exocytotic processes (377). This latter study provided the first functional evidence for the role of Rab proteins in CD36 trafficking. Moreover, Rab11 appears to play a similar role in GLUT4 and CD36 translocation. Whether the role of Rab5 in GLUT4 translocation could be extrapolated to the translocation of CD36 or other fatty acid transporters remains to be determined. It is, however, unlikely that Rab4 is involved in translocation of fatty acid transporters, as this Rab member is associated with the insulin-responsive GLUT4 storage compartment (431), which is devoid of CD36 (see sect. vA1).

C) COAT PROTEINS. Essentially nothing is known about the involvement of coat proteins in CD36 trafficking, except that caveolin-3 has been colocalized with CD36 at the sarcolemma $(236,464)$. Its stimulatory role, if any, in fatty acid uptake has already been discussed (see sect. IIID). Another mechanism by which caveolin-3 could be positively involved in fatty acid uptake is that it clusters t-SNARE proteins at the cell surface and, as a result, could form a docking station for CD36 vesicles. This, however, remains to be determined.

D) OTHER TRAFFICKING PROTEINS. Adaptor proteins assist in the assembly of coat and SNARE proteins into fission and fusion complexes as well as modulate the function and intracellular localization of Rab proteins. These adaptors also add an extra level of specificity for delivering the cargo to the appropriate subcellular address. It is beyond the scope of this review to discuss the 
manner in which all these adaptors are involved in GLUT4 translocation. Generally, the roles of these proteins in the translocation of fatty acid transporters have not yet been assessed, although evidence is beginning to emerge that selected adaptors also function in CD36 translocation. One of these accessory proteins is Munc18c, a member of the Sec1p-like/Munc18 family, which functions in the transition of syntaxins (tSNAREs) into their open and closed states (111). Munc18c forms a complex with syntaxin-4 (175) and is known to be involved in GLUT4 translocation, as has been shown in cell lines (429) and in skeletal muscle from heterozygous Munc18c knockout mice (319). In cardiac myocytes from heterozygous Munc18c knockout mice, Munc18c appeared to play a similar role in CD36 and GLUT4 translocation, induced by either insulin or AMPK activation, in that a $\sim 50 \%$ deletion did not affect fatty acid or glucose uptake induced by each of these metabolic stimuli. Hence, Munc18c appears to be necessary, but not rate-limiting, in cardiac fatty acid and glucose uptake under basal and stimulated conditions (154). In addition, two adaptors for Rab11 have been studied for their role in CD36 recycling (377). Both adaptors belong to a family of Rab11 interacting proteins (FIPs) with a conserved Rab11-binding domain. The first, FIP2, functions as an adaptor for direct interaction of Rab11 with the motor protein myosin $\mathrm{Vb}$ (158), and the second, Rip11, colocalizes with Rab11 in endosomal membranes (339). Silencing of FIP2 in H9c2 cells enhanced the surface abundance of both GLUT4 and CD36, while silencing of Rip11 selectively enhanced surface CD36 (377). Hence, Rip11 is normally involved in the intracellular retention of CD36, but not of GLUT4, and this allows Rab11 to discriminate between CD36 and other cargo for trafficking purposes. Interestingly, in adipocytes Rip11 has been reported to be involved in insulin-stimulated GLUT4 translocation possibly through interaction with AS160 (473), indicating that certain trafficking proteins fulfill different roles in different tissues.

Taken together, it is clear that at present the amount of information about signaling and trafficking processes in translocation of fatty acid transporters is at its infancy, yet information about these events is beginning to accumulate. It appears that the trafficking machinery is capable of selectively recruiting fatty acid transporters from either insulin-responsive stores or contraction-responsive stores, through as yet unidentified trafficking proteins that are specifically dedicated to either of these stores. Knowledge about the protein composition of the GLUT4 translocation machinery will provide a framework for examining whether these proteins are also involved in translocation of fatty acid transporters. The identification of novel proteins involved in signaling and trafficking of fatty acid trans- porters would provide strategies to pharmacologically regulate substrate fluxes. This may be especially important for the treatment of metabolic diseases characterized by altered fatty acid metabolism in heart and skeletal muscle (see sect. vIIIB).

\section{CHRONIC PHYSIOLOGICAL REGULATION OF FATTY ACID TRANSPORTERS}

The expression and functioning of the membrane fatty acid transporters is influenced by common physiological stimuli. While in the previous section we focused on acute effects $(<30 \mathrm{~min})$, which can alter the subcellular localization of fatty acid transporters, in this section we will discuss our current understanding of the longterm effects, i.e., involving de novo protein synthesis. These long-term effects are mediated by specific transcription factors and coactivators.

Importantly, it should be remembered that proteins, not mRNAs, are the functional unit for physiological processes. The common assumption that fatty acid transporter mRNAs are a suitable index of their protein product is problematic. For example, changes in fatty acid transporter mRNAs frequently do not correlate either with changes in their protein expression or with changes in the rate of fatty acid transport in many experimental models $(27,28,44,71,273,277,278,281,443,479)$, indicating a prominent role for posttranscriptional processes. These studies also illustrate that extrapolation of mRNA data to functional consequences can result in interpretive and conceptual errors. Therefore, it is preferable to examine fatty transporters at the level of their protein expression, although even such data are limiting given that fatty acid transporters can traffic between subcellular compartments (see sect. Iv). After all, just as for GLUT4, the functional pools of fatty acid transporters are those at the plasma membrane.

\section{A. Regulation of Fatty Acid Transporter Expression}

Like many proteins involved in lipid transport and metabolism, the expression of most, if not all, membrane fatty acid transporters is under the transcriptional control of nuclear peroxisome proliferator-activated receptors (PPAR) in a tissue-specific manner. Depending on the presence of (one or more of) the three PPAR subtypes, $\alpha$, $\beta / \delta$, and $\gamma$, cells will respond to specific PPAR agonists by transcriptionally upregulating the expression of fatty acid transporters. For instance, synthetic agonists for PPAR $\alpha$ (known as fibrates) induce CD36 expression in the heart (19), while CD36 expression is significantly decreased in hearts and muscle of the PPAR $\alpha$ null mouse (307). Because PPAR $\gamma$ is expressed in skeletal muscle but virtually 
absent in heart (19), CD36 expression was found sensitive to regulation by PPAR $\gamma$ agonists in human skeletal muscle (70) but not in rat cardiac myocytes (454). Fatty acids and selected fatty acid metabolites are the physiological ligands for the PPARs, allowing mammalian cells to upregulate fatty acid transporters and the lipid metabolic machinery in case of increased exposure to fatty acids, so to create a vicious cycle of increased exposure and utilization (for review, see Refs. 291, 398).

While initial studies had concluded that the promoter region of CD36 contains a peroxisome proliferator-responsive element (PPRE) (433), subsequent studies revealed the absence of PPRE in the responding upstream promoter region and showed that transcriptional activation of the CD36 gene by PPAR ligands is indirectly dependent on PPAR (369). This would also explain the delayed transcriptional activation of CD36 expression by PPAR ligands, compared with other PPAR target genes (369). With respect to the other fatty acid transporters, a functional PPRE has been identified in the promoter region of FATP1 (129) and likely is also present in the promoter of the other FATPs. In line with this, PPAR activators were found to coordinately regulate the expression of FATP1 and acyl-CoA synthetase in rat tissues (293). The presence of a PPRE in the promoter of $\mathrm{FABP}_{\mathrm{pm}}$ has not been reported, but is likely as PPAR $\gamma$ activation induced the expression of $\mathrm{FABP}_{\mathrm{pm}}$ in rat skeletal muscle (22).

More recent studies have disclosed that overexpression of the PPAR $\gamma$ coactivator $1 \alpha(\mathrm{PGC}-1 \alpha)$ increased the expression of CD36 $(23,82)$, as well as GLUT4 (23), in rat muscle, together with an increase in mitochondrial fatty acid oxidation (22). Finally, CCAAT/enhancer binding protein $\alpha(\mathrm{C} / \mathrm{EBP} \alpha)$ was found to regulate $\mathrm{CD} 36$ gene expression at the transcriptional level (341), but the role of this transcription factor most likely is restricted to adipocytes, especially during the differentiation of these cells.

Taken together, fatty acids and specific metabolites are now recognized to modulate the expression of multiple genes, notably those involved in fatty acid metabolism, in a PPAR-dependent manner. Fatty acids as signaling molecules thereby "prepare" the cell for the uptake and utilization of larger amounts of fatty acids. Fatty acid transporters take part in this process in a special fashion as they could promote a positive-feedback loop to further increase gene transcription.

\section{B. Effects of Development, Ageing, and Gender}

\section{Development and ageing}

The oxidation capacities and mitochondrial enzymes of heart and muscle increase quickly during development, reaching adult values at 15-20 days postnatally in heart (144) and by 35 days in skeletal muscle (47). A similar rapid adaptation (days 10-35) was observed in CD36 protein expression in rat heart and muscle, while $\mathrm{FABP}_{\mathrm{pm}}$ protein expression was unaltered in the heart and was reduced somewhat in muscle (47). Thereafter, CD36 expression and mitochondrial enzyme activity remained invariant from 5 to $52 \mathrm{wk}$ (47). The increases in fatty acid transport in the transition from the neonatal period (day 10) to the adult stage was attributable to concomitant increases in plasmalemmal CD36 in heart, and to plasmalemmal CD36 and $\mathrm{FABP}_{\mathrm{pm}}$ in muscle.

In older rats (15-24 mo vs. 5 mo), fatty acid oxidation was reduced while esterification was increased (441). The concurrent changes in fatty acid transporters were not detailed except to note that $\mathrm{FABP}_{\mathrm{pm}}$ expression was increased (441). Similarly, in hearts of aged mice (52 wk), there is a twofold increase in intramyocardial lipid accumulation, as well as a $\sim 50 \%$ reduction in fatty acid oxidation (254), which is accompanied by a dramatic increase in CD36 (4-fold) in these aged murine hearts compared with young hearts. This suggests that CD36 is a mediator of multiple metabolic, functional, and structural alterations in the aged heart, particularly since hearts from aged CD36 null mice were found to have lower intramyocardial lipid concentrations and enhanced cardiac function compared with the aged wild-type mice (254).

Taken together, it appears that myocardial fatty acid uptake increases to a higher level at both ends of the life span, i.e., perinatally and during ageing, effects that appear to be mediated in part by the upregulation of CD36. While in the neonate the increase in CD36 would seem to be positively associated with the capacity to oxidize fatty acids, it appears that in the aged animals the further upregulation of CD36 allows the uptake of fatty acids to outpace the capacity for fatty acid oxidation. This then leads to deleterious metabolic consequences in heart and muscle of aged animals, which resemble those observed in insulin resistance and type 2 diabetes in which CD36mediated fatty acid transport also exceeds the capacity for oxidation (see sect. $\mathrm{vII} B$ ).

\section{Gender differences}

Fatty acid metabolism has long been suspected to be greater in females than in males. This gender difference has been addressed in a recent review, particularly as it applies to skeletal muscle triacylglycerol esterification and oxidation (242). Although a number of fatty acid transporters are coexpressed in rat and human muscle, including $\mathrm{FABP}_{\mathrm{pm}}$, CD36, FATP1, and FATP4 (45, 46, 219, 445), it has been difficult to demonstrate that there are gender differences in fatty acid transporters in skeletal muscle. For example, CD36 protein expression was somewhat higher in women than in men (244), but this was not observed in another study (389). Gender differences have 
not been observed in $\mathrm{FABP}_{\mathrm{pm}}$ protein expression or FATP1 mRNA $(244,384)$. However, there appear to be gender-related sensitivities in CD36 responses to fatty acid infusion, since in male, but not female rats, fatty acid infusion induced insulin resistance and reduced muscle CD36 total protein by 50\% (189). Similarly, it has been observed that $\mathrm{FABP}_{\mathrm{pm}}$ protein expression is increased in endurance-trained men but not endurance-trained women (244). Some of these discrepant findings between men and women may be related to difficulty in matching the sexes appropriately and/or the criteria that are employed for matching individuals.

\section{Effects of Fasting, Hormones, and Exercise Training}

\section{Fasting}

Several studies have shown that fasting alters fatty acid metabolism in heart and skeletal muscle. For example, short-term fasting $(12-48 \mathrm{~h})$ increased $\mathrm{FABP}_{\mathrm{pm}}$ protein at the plasma membrane by $60 \%$ (444). Similarly, $48 \mathrm{~h}$ of fasting increased CD36 expression in the heart $(\sim 2$ fold) (455) and in skeletal muscle ( $20 \%)$. The fastinginduced upregulation of CD36 mRNA and protein, as well as other genes involved in lipid metabolism, is AMPK 33 -dependent (269).

\section{Insulin}

In cardiac myocytes, insulin not only (acutely) induces the translocation of CD36, but also relatively rapidly $(<2 \mathrm{~h})$ increases the expression of CD36 protein, but not $\mathrm{FABP}_{\mathrm{pm}}$ (72). Similarly, CD36 protein content was increased 1.5-fold in human muscle after $3 \mathrm{~h}$ of insulin stimulation (94). In the studies in perfused hearts and cardiac myocytes there were similar insulin-induced increments in CD36 protein expression (cardiac myocytes $+43 \%$; Langendorff-perfused hearts $+32 \%$ ) and in plasmalemmal protein content $(+29 \%)$ and rates of fatty acid transport (+34\%) (72). Thus CD36 expression is highly responsive to insulin stimulation, although in insulin-resistant individuals with increased circulating concentrations of insulin, only the plasmalemmal content of CD36 expression is increased, not total CD36 protein expression (48).

\section{Leptin}

It is well documented that leptin acutely increases fatty acid utilization by activating AMPK in skeletal muscle (302, 309, 310, 403-406). Leptin appears to induce the translocation of CD36 from an intracellular depot to the plasma membrane $(305,324)$, thereby increasing fatty acid uptake and providing the necessary substrate for fatty acid oxidation. In contrast, prolonged leptin infusion
(2 wk) downregulated $\mathrm{FABP}_{\mathrm{pm}}$ and CD36 protein expression as well as their plasmalemmal contents in skeletal muscle, resulting in a parallel reduction in fatty acid transport (404). This long-term effect of leptin may have been a compensatory response. Similarly, in adipocytes of $o b / o b$ mice, prolonged leptin treatment (21 days) reduced $\mathrm{FABP}_{\mathrm{pm}}$ and CD36 mRNAs and normalized fatty acid uptake (121). Clearly, leptin exerts profound effects on fatty acid transport and transporters, although the acute and chronic effects of this adipokine differ.

\section{Exercise training}

Short-term electrically stimulated muscle contraction, and likely also acute volitional exercise, have been described in section IV to stimulate both fatty acid and glucose utilization via translocation of GLUT4, CD36, FABP $_{\text {pm }}$, FATP1, and FATP4 $(44,219,277,443)$ but not FATP6 (219) to the plasma membrane. In contrast, it is well known that endurance exercise training shifts fuel selection both at rest and during exercise towards fatty acid oxidation and esterification, as training-induced adaptive responses include increased activity of enzymes involved in fatty acid oxidation and esterification, as well as intramuscular triacylglycerol accumulation $(40,113$, 196, 215, 242, 374, 457). Because exercise training does not increase the circulating concentrations of fatty acids, it seems feasible that an increase in fatty acid transport proteins supports the enhanced capacity for fatty acid metabolism in trained skeletal muscle.

In rats, exercise training failed to alter the expression of either CD36 or $\mathrm{FABP}_{\mathrm{pm}}$ total proteins in the heart despite the fact that fatty acid oxidation was increased (61). This may suggest perhaps that these proteins were redistributed in the cell to increase the plasmalemmal content, as this has previously been shown to account for the increased fatty acid oxidation in murine $d b / d b$ hearts, in which fatty acid oxidation is markedly increased (65).

Increasing muscle activity with exercise training (447) or 7-day chronic low-frequency stimulation of the peroneal nerve $(41,252)$ increased both CD36 and $\mathrm{FABP}_{\mathrm{pm}}$ protein expression and their plasma membrane contents, as well as increasing the sarcolemmal transport rate of fatty acids (41, 252, 447). The increase in fatty acid transport (1.9fold) matched the increase in fatty acid metabolism (1.9fold, sum of oxidation and esterification) (41). Induction of muscle inactivity (7-day denervation) lowered rates of fatty acid transport, but this was due to a reduction in plasma membrane CD36 and $\mathrm{FABP}_{\mathrm{pm}}$, while there was no change in their total protein expression levels (252) (Fig. 6). Taken together, these findings indicate that fatty acid transport can be regulated by several mechanisms, i.e., 1) by altering the total protein expression of CD36 and $\mathrm{FABP}_{\mathrm{pm}}$ and their contents at the plasma membrane (exercise training or chronic muscle stimulation), or 2) by altering their 
presence at the plasma membrane, without altering protein expression (denervation). Indeed, the functional pool of fatty acid transporters are those at the plasma membrane, since the up- and downregulation in fatty acid transport in chronically stimulated and 7-day denervated muscle correlated highly with the plasmalemmal contents of CD36 and FABP $_{\text {pm }}$ (252) (Fig. 6).

Exercise training studies in humans have shown variable responses with respect to adaptive changes in the protein expression of CD36 and $\mathrm{FABP}_{\mathrm{pm}}$. In some studies exercise training has increased CD36 but not $\mathrm{FABP}_{\mathrm{pm}}$ (63\% $\mathrm{VO}_{2 \text { peak }}$; Ref. 442). Others have shown, applying a one-leg knee extension training model in which the contralateral muscle serves as control, that $\mathrm{FABP}_{\mathrm{pm}}$ protein expression is increased (CD36 was not measured; Ref. 243). These different effects observed may be exerciseintensity dependent, as with high-intensity exercise training $\left(90 \% \mathrm{VO}_{2 \text { peak }}\right)$ there was only an increase in $\mathrm{FABP}_{\mathrm{pm}}$ but not CD36 (423), while with very-high-intensity exercise $\left(>100 \% \mathrm{VO}_{2 \text { peak }}\right)$ that is largely dependent on glycogen utilization no changes were observed in either CD36 or $\mathrm{FABP}_{\mathrm{pm}}$ protein expression, but increases did occur in other transport proteins (GLUT4, MCT-1 and -4; Ref. 62). Upregulation of fatty acid transporters CD36 and $\mathrm{FABP}_{\mathrm{pm}}$ may also be influenced by the dietary state during training. For example, providing a high carbohydrate-rich breakfast before training failed to result in any traininginduced changes in CD36 and $\mathrm{FABP}_{\mathrm{pm}}(100)$. This contrasted markedly with training in the fasted state, when the demands on fatty acid metabolism are greater, as this increased $(\sim 20 \%)$ both CD36 and $\mathrm{FABP}_{\mathrm{pm}}$ protein expression coordinately $(r=0.63)$ (100). It may be important in future studies to determine whether fatty acid transporters have been relocated to the plasma membrane and/or to the mitochondria, in the absence of any changes in their expression. Subcellular relocation of fatty acid

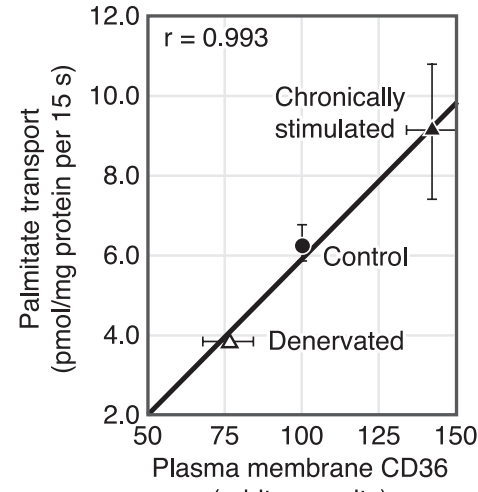

(arbitrary units)

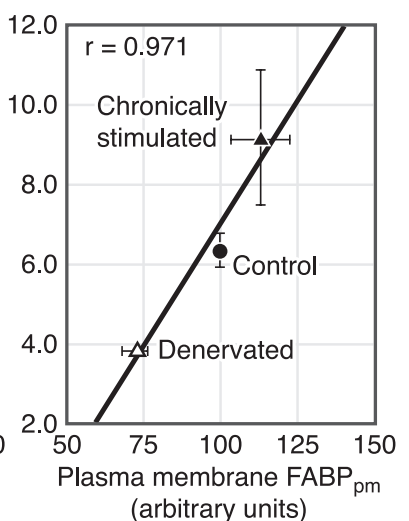

(arbitrary units)
FIG. 6. Chronically altered muscle contractile activity affects the rate of fatty acid uptake and plasmalemmal CD36 and $\mathrm{FABP}_{\mathrm{pm}}$. Rat hindlimb muscles were chronically stimulated ( 7 days) or denervated ( 7 days) whereafter fatty acid transport into giant sarcolemmal vesicles and plasmalemmal CD36 (left) and $\mathrm{FABP}_{\mathrm{pm}}$ (right) content were determined. FATPs were not studied. [Redrawn from Koonen et al. (252).] transporters without a change in their protein expression have been observed in denervated muscle (see above) (252) and in muscles from obese animals (273) and humans (48) (see sect. VII $B$ ).

\section{ALTERATIONS IN FATTY ACID TRANSPORTERS IN DISEASE}

In view of the general significance of membrane fatty acid transporters for whole body lipid metabolism and specifically their role in the acute regulation of cellular fatty acid uptake in muscle and heart, it is to be expected that changes in cellular lipid metabolism such as occur in disease states will be accompanied by concomitant adaptations in the expression and/or subcellular location of selected fatty acid transporters. On the other hand, changes in fatty acid transporter content or functioning, as may be caused by nutritional, hormonal, or pharmacological stimuli, will likely have an impact on whole body lipid metabolism. Examples of both such alterations, with a primary focus on heart and muscle, will be discussed in this section. These findings also begin to suggest that membrane fatty acid transporters may be exploited as therapeutic targets.

\section{A. Cardiac Hypoxic Disease and Heart Failure}

The heart can use a variety of metabolic substrates to cover its demand for energy, and it has the capacity to rapidly switch among these substrates in response, e.g., to changes in their supply, actions of hormones and cytokines, and the availability of oxygen (400). In normal conditions, cardiac metabolic energy is provided from the oxidation of long-chain fatty acids (40-60\%), glucose (30-50\%), and lactate (10\%). In hypoxic conditions, as caused acutely by an ischemic insult or myocardial infarction, the heart switches to the substrate with the highest oxygen efficiency, i.e., carbohydrates, provided from glucose and endogenous cardiac glycogen. During reperfusion, when oxygen supply is reinstated, there is a progressive increase in the contribution of fatty acids to ATP production. In chronic cardiac disease and heart failure, the changes in substrate preference are more permanent and are accompanied by alterations in the expression of metabolic enzymes and proteins. Such chronic changes might influence the so-called metabolic flexibility of the heart, i.e., the ability to switch to other energy substrates and to better withstand an acute metabolic stress $(140,400)$.

\section{Acute changes}

The acute switch in substrate utilization that occurs during short-term hypoxia, i.e., an increased contribution 
from carbohydrates and decreased contribution from fatty acids to energy production, appears not be accompanied by a decreased sarcolemmal presence of fatty acid transporters, which would be expected to lower fatty acid uptake. Instead, studies with rat myocytes and perfused hearts show that acute hypoxia activates AMPK, which induces the translocation of both CD36 and $\mathrm{FABP}_{\mathrm{pm}}$ to the sarcolemma leading to a concomitant increase in the rate of fatty acid uptake (74). Because of the reduced rate of fatty acid oxidation, a large portion of the incoming fatty acids are redirected into intracellular lipid pools. These observations reveal a role for CD36 and $\mathrm{FABP}_{\mathrm{pm}}$ in intramyocardial lipid accumulation that occurs in the failing heart and that is associated with cardiac contractile dysfunction (380) (see sect. $\mathrm{VI} B$ ).

During reperfusion following an ischemic insult, there is a shift towards increased fatty acid utilization. Whether CD36 serves a beneficial or detrimental role during this transition has been studied by two groups of investigators. Irie et al. (216) reported that isolated working hearts from CD36 null mice showed a $40 \%$ lower postischemic recovery of cardiac function compared with hearts from wild-type littermates. Hearts from mice reexpressing CD36 in heart and skeletal muscle under the muscle creatine kinase promoter showed an ischemic tolerance comparable to that of wild-type hearts, further indicating that the decreased tolerance in CD36 null mice is directly caused by CD36 deficiency (216). Dietary intervention with mediumchain fatty acids to circumvent the CD36-mediated uptake resulted in normalization of the ischemic tolerance of CD36 null hearts. These observations led the authors to suggest that the presence of CD36 is crucial to upregulating fatty acid uptake and cardiac energy production during the transition from ischemia to reperfusion and, therefore, that CD36 is a determinant of ischemic tolerance of the heart. In contrast, a subsequent comparable study (258) reported that the postischemic recovery of CD36 null hearts is not different from that of wild-type hearts and that during reperfusion accelerated glucose oxidation can compensate for the loss of fatty acid-derived ATP (258) (Fig. 7). Most likely, these conflicting observations are explained by differences in energetic status of the hearts before ischemia, namely, there was a $40 \%$ lower content of both ATP and glycogen in the CD36 null hearts relative to wild-type hearts in one study (216) but not the other (258). Taken together, these two studies suggest that a deviation in the normal contributions of glucose and fatty acid oxidation to cardiac energy production, for instance, towards a markedly increased dependence on glucose oxidation as seen in CD36 null hearts (258), will not affect cardiac ATP production when adequately perfused. In contrast, such a chronic change in cardiac substrate preference renders the heart more susceptible to stressors like an
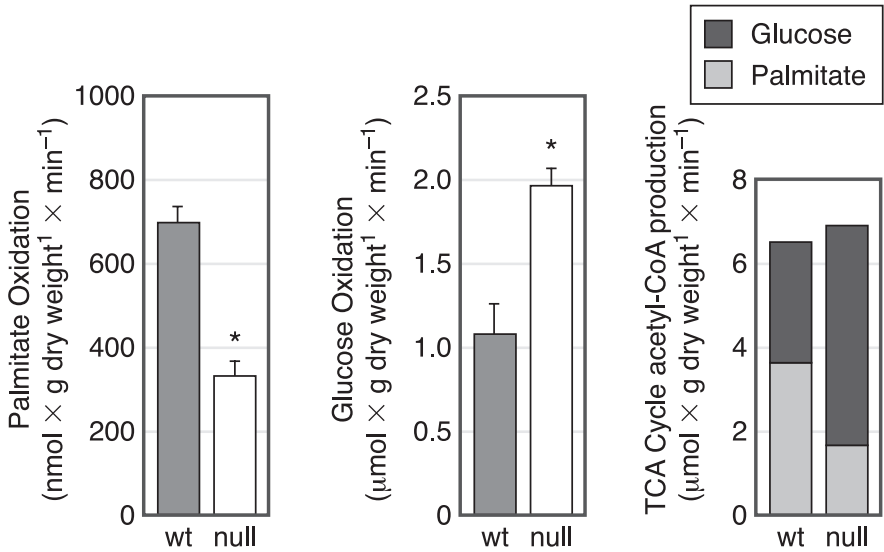

FIG. 7. Changes in substrate utilization in hearts from CD36 null mice. Rates of palmitate oxidation (left), glucose oxidation (middle), and tricarboxylic acid (TCA) cycle acetyl-CoA production (right) were determined in wild-type and CD36 null mouse hearts perfused as working hearts with $1.2 \mathrm{mM}$ palmitate and $5 \mathrm{mM}$ glucose. TCA cycle activity was calculated from the rates of palmitate and glucose oxidation, using $8 \mathrm{~mol}$ acetyl-CoA for every $1 \mathrm{~mol}$ palmitate oxidized and 2 mol acetylCoA for every $1 \mathrm{~mol}$ glucose oxidized. The lower palmitate oxidation seen in hearts from CD36 null mice is compensated by an increase in glucose oxidation so that these hearts are not energetically compromised. [Redrawn from Kuang et al. (258), with permission from the American Heart Association.]

ischemic insult when a lower ATP production rate may depress cardiac function. Furthermore, substrate transporters provide dynamic regulation of substrate uptake and thus add to the metabolic flexibility of the heart.

\section{Chronic changes}

The development of cardiac hypertrophy and heart failure is associated with changes in cardiac substrate preference, namely, glucose utilization is increased at the expense of fatty acids $(10,400)$. In the postinfarction rat heart, this change in substrate preference is reflected in an upregulation of GLUT1 and downregulation of genes of lipid metabolic enzymes and of fatty acid transporters (181, 359). Specifically, the myocardial contents of $\mathrm{FABP}_{\mathrm{pm}}$, CD36, FATP1, and FATP6 protein expression were each reduced with a parallel decrease in palmitate oxidation rate and in cardiac ejection fraction (181). These data suggest that fatty acid transporters may be involved in the transition away from fatty acid metabolism in heart failure, but it should be noted that the subcellular localization of the transporters was not examined in either of these studies.

Likewise, fatty acid transporter deficiency, which will limit cardiac fatty acid utilization and elicit an increase in glucose utilization, is associated with the development of cardiac hypertrophy $(216,313)$. This parallels the effect of pharmacological inhibition of mitochondrial $\beta$-oxidation, which also leads to increased glucose utilization and ultimately to cardiac hypertrophy (54, 360). 
For example, CD36 null mice on a regular diet develop cardiac hypertrophy $(216,313)$, but interestingly, FATP1 null mice do not (up to 15 mo of age) (245). Chronic (12 wk) intraperitoneal administration of $\mathrm{SSO}$, a selective inhibitor of CD36-mediated fatty acid uptake (93, 153), caused cardiac hypertrophy (260). Conversely, aged ( $>1 \mathrm{yr}$ ) mice developed cardiac hypertrophy in the face of a greater than fourfold increase in total myocardial CD36 protein expression in aged animals (254), but in this study neither sarcolemmal CD36 nor fatty acid uptake was determined, hampering the interpretation of the data. Nevertheless, when taken together, the above findings suggest that fatty acid transporters are mediators of substrate-induced cardiac remodeling.

A role for CD36 deficiency in the development of cardiac hypertrophy was suggested from studies with a strain of spontaneously hypertensive (SHR) rats maintained in North America $(7,157)$. However, it appears that these animals are not CD36 deficient, and fatty acid uptake is not compromised (43) (see sect. $\mathrm{vII} B$ ). Thus cardiac hypertrophy in this model is attributable to other factors.

\section{Human studies}

There is some evidence to suggest that the link between CD36 expression and cardiac hypertrophy and possibly tolerance to ischemia, as observed in rodent models, can also be extended to humans. A Japanese study revealed that $\sim 40 \%$ of patients with hypertrophic cardiomyopathy carry mutations in CD36 leading to a negligible or markedly reduced CD36 protein expression (320, 425). Mutations leading to CD36 deficiency are rare in Caucasian populations $(<0.3 \%)$, but these are at least 10 times more frequent (3-6\%) in Asian, African, and AfricanAmerican populations $(98,262,271)$. These CD36 mutations were shown to lead in almost all cases to severe reductions in myocardial long-chain fatty acid uptake $(320,425)$, which metabolic change may have triggered the hypertrophic response as seen in the animal studies described above. On the other hand, a subsequent study (424) showed that patients with a total defect in myocardial fatty acid uptake, which in all cases was associated with the absence of CD36, do not always develop hypertrophic cardiomyopathy (424). This latter finding and the broad spectrum of clinical manifestations of hypertrophic cardiomyopathy may explain why another study reported that the incidence of CD36 deficiency in hypertrophic cardiomyopathy patients is not higher than in the general population (311). Clearly, the development of heart disease in humans is complex, and the association between CD36 expression and heart function and failure needs further exploration, whereby special attention should be given to the functional presence of CD36 on the sarcolemma and its association with mitochondria.

\section{B. Insulin Resistance and Type 2 Diabetes}

Insulin resistance and type 2 diabetes are associated with changes in lipid metabolism. For example, there is a strong association between skeletal muscle insulin resistance and 1) plasma fatty acid concentrations (36-38) and 2) intramuscular triacylglycerol depots (220, 221, 256, $326,409)$. However, intramuscular triacylglycerol depots are an indirect marker of insulin resistance, since the more soluble lipid metabolites such as ceramides, diacylglycerols, and long-chain fatty acyl-CoAs interfere with the postreceptor insulin signaling cascade $(77,78,90,193$, 194, 410, 421). This excessive accumulation of intramyocellular fatty acids and their metabolites has been referred to as lipotoxicity and is a main contributor to the pathophysiology of insulin resistance and dysfunctioning of heart and skeletal muscle $(194,451)$. The pathological state of the lipid-overloaded, insulin-resistant, and failing heart is commonly referred to as diabetic cardiomyopathy $(1,400)$. Several recent reviews discuss in detail the defects in postreceptor insulin signaling mechanisms (1, $194,207,428)$ that are at the root of fatty acid-induced insulin resistance in skeletal muscle and the heart in obesity and type 2 diabetes.

Fatty acids that are taken up into heart and skeletal muscle are primarily oxidized or stored as triacylglycerols. As long as the fatty acid uptake and partitioning remains appropriately balanced, metabolic dysregulation does not occur. However, these processes are unbalanced toward lipid accumulation in insulin-resistant skeletal muscle $(18,48,164)$ and the heart $(322,380)$. This increase in intracellular lipid accumulation would not seem to be simply attributable to a concomitant reduction in (mitochondrial) fatty acid oxidation as has been speculated (238), since there is no consistent evidence in support of this notion (for review, see Ref. 195). For example, in heart and skeletal muscles of high fat fed rats, $d b / d b$ mice, obese Zucker rats, and ZDF rats fatty acid oxidation is either reduced slightly $(18,164,322)$, unaltered $(387,482)$, or increased $(65,66,91,446)$. In muscle from obese humans, a reduction in skeletal muscle fatty acid oxidation is at times observed $(202,210,246,430)$, but not always $(48,405)$. In individuals with type 2 diabetes, whole muscle fatty acid oxidation also appears to be reduced (18), and basal mitochondrial oxidative phosphorylation was reduced in insulin-resistant offspring of patients with type 2 diabetes (331). In obese individuals, the reduction in fatty acid oxidation appears to be correlated with the extent of obesity (BMI) (430) and has also been attributed to a reduced mitochondrial content (202, $246,384)$, as fatty acid oxidation by isolated mitochondria of obese individuals was normal (202) and not impaired as had been suggested previously based on indirect assessment of mitochondrial fatty acid oxidation (237, 355). Similarly, in individuals with type 2 diabetes, reductions 
in muscle fatty acid oxidation and oxidative phosphorylation appear to be a result of reduced mitochondrial content, possibly as a result of diminished physical activity, rather than an intrinsic defect in their mitochondria $(50,101,304,331,342)$, especially since in isolated mitochondria respiratory function, mitochondrial citrate synthase, and $\beta$-HAD activities were normal, as was the rate of palmitoyl-L-carnitine oxidation $(50,304)$. It has been suggested recently that obesity-related insulin resistance in skeletal muscle is associated with an inability of mitochondria to oxidize the excess influx of fatty acids, which leads to an accumulation of intramuscular lipids (255). We (197) have examined this question as described further in section $\mathrm{VII} B 2$.

In conclusion, observations in some studies that mitochondrial fatty acid oxidation may be increased at times suggests that factors other than simply a reduction in fatty acid oxidation can contribute to the intramuscular lipid accumulation, particularly 1) an increased delivery of circulating fatty acids, such as is frequently observed in obesity, and/or 2) an increased rate of in fatty acid transport, facilitated by an increase in fatty acid transporters. Importantly, in cases that reductions in fatty acid oxidation in insulin-resistant skeletal muscle were reported, these reductions reflect reductions in mitochondrial content rather than impairments in the intrinsic ability of mitochondria to oxidize fatty acids.

\section{Permanent subcellular relocation of fatty acid transporters}

A) ANIMAL MODELS OF INSULIN RESISTANCE. Studies examining the rates of fatty acid transport have been performed in animal models of high-fat diet-induced insulin resistance $(182,322)$, in obese (Zucker obese $f a / f a)(197,273)$ and type 2 diabetic rats (Zucker diabetic fatty, ZDF) (27, $71,91)$, and in murine models of obesity $(28,150)$. These studies have shown that fatty acid uptake rates were increased in key metabolic tissues, including 1) hearts of obese Zucker rats (40-90\%; Refs. 27, 91, 273), ZDF rats (120\%; Ref. 27), and rats fed a high-fat diet (43\%; Ref. 322); and 2) skeletal muscle of high fat fed rats (40-80\%; Ref. 182), obese Zucker rats (80\%; Ref. 273), in red but not white muscle of ZDF rats (+66-99\%; Ref. 71), and in rats fed a high-sucrose diet during the suckling period (50\%; Ref. 211). Thus, in all models of insulin resistance, fatty acid transport is upregulated in heart and skeletal muscle. This upregulation is already present in muscles of 6-wkold ZDF rats, before the onset of type 2 diabetes (71). It appears that upregulation of fatty acid transport is a predisposing factor to obesity and insulin resistance. However, the underlying mechanisms promoting fatty acid transport appear to differ depending on the severity of insulin resistance (see below).
B) MODEST AND MODERATE INSULIN RESISTANCE. In models of modest (high-fat diet; Refs. 182, 322) to moderate insulin resistance (obese Zucker rats; Refs. 91, 164, 273), the increased rates of fatty acid transport in heart and muscle in general were not related to the increased expression of fatty acid transporter proteins (CD36 and $\mathrm{FABP}_{\mathrm{pm}}$ ) in these tissues $(91,211,273,322)$. However, there was a marked increase in the content of the functional pool of fatty acid transporters, i.e., those at the plasma membrane in heart (CD36, +60-74\%; $\left.\mathrm{FABP}_{\mathrm{pm}},+50 \%\right)(91,273)$ and in skeletal muscle (CD36, +33-80\%; FABP $\left.{ }_{\mathrm{pm}}, 0-14 \%\right)$, (164, 211, 273). This "permanent relocation" of CD36 to the plasma membrane in skeletal muscle has been confirmed in more recent studies, which also demonstrated that such changes were not observed for plasmalemmal $\mathrm{FABP}_{\mathrm{pm}}$, FATP1, and FATP4 (197). Thus these studies have revealed a previously unknown mechanism that increases fatty acid uptake in modest and moderate models of insulin resistance. Specifically, CD36 and $\mathrm{FABP}_{\mathrm{pm}}$ are permanently relocated from their intracellular depots to the cell surface in heart $(91,273)$, while in skeletal muscle such a permanent relocation was mainly observed for CD36 only, not $\mathrm{FABP}_{\mathrm{pm}}$, FATP1 or FATP4 $(164,197,273)$.

C) SEVERE INSULIN RESISTANCE. In ZDF rats, which progress rapidly from insulin resistance (week 6) to severe type 2 diabetes (week 24$)(71,466,467)$, there was an increase in the rate of fatty acid transport and the CD36 protein expression and plasmalemmal content compared with age-matched, nondiabetic animals (71). Similarly, in female ZDF rats, which develop diabetes when fed a high-fat diet, there was also an increase in muscle CD36 protein expression and plasmalemmal content (387). However, concurrent treatment with metformin or exercise impaired the increase in CD36 protein expression and plasmalemmal content, and this was associated with reductions (relative to the high-fat diet) in circulating glucose and in intramuscular ceramide and diacylglycerol contents. This study (387) begins to suggest that reducing plasmalemmal CD36 reduces the severity of intramuscular lipid accumulation and hyperglycemia.

D) METABOLIC EFFECTS OF IMPAIRED CD36 TRANSLOCATION ON FATTY ACID METABOLISM. Presumably, the insulin resistance in CD36 signaling/trafficking accounts, in part, for the inability of insulin to further stimulate triacylglycerol esterification in obese Zucker rat heart and muscle, beyond the already increased rates observed under basal conditions (91, 164). This, however, is not apparent during contraction (muscle) or AMPK activation (heart), as the normal $\mathrm{FABP}_{\mathrm{pm}}$ translocation (164), along with the already upregulated plasmalemmal CD36 $(91,164)$, seems to provide the needed fatty acid transport capacity to upregulate fatty acid oxidation rates in obese animals. This begins to imply that an increase in both plasmalemmal CD36 and $\mathrm{FABP}_{\mathrm{pm}}$ contribute to the upregulation in contraction- 
stimulated fatty acid oxidation. Indeed, some tentative preliminary evidence for this has been shown in skeletal muscle into which CD36 and $\mathrm{FABP}_{\mathrm{pm}}$ were cotransfected (314).

\section{Key role of CD36 in insulin resistance}

In the type 2 diabetic heart ( $d b / d b$ mice), the increased expression $(+50 \%)$ and plasmalemmal content of CD36 ( $+75 \%)$, and possibly the small change in plasmalemmal $\mathrm{FABP}_{\mathrm{pm}}(+18 \%)$, were positively correlated with the increased rates of fatty acid esterification $(\sim 2$-fold $)$. All other parameters involved in regulating lipid metabolism (CPT-I activity, CPT-I sensitivity to inhibition by malonylCoA, UCP3 expression) were either unaltered or reduced (AMPK activity) (65). This suggests that in rodent models deficient in leptin signaling, alterations in sarcolemmal fatty acid transporters rather than alterations in mitochondrial $\beta$-oxidation are responsible for cardiac lipid accumulation and, subsequently, loss of cardiac function.

More direct evidence for the role of CD36 in increased fatty acid esterification and lipid accumulation in the insulin resistant heart was obtained with pharmacological and genetic experiments to block CD36. In cardiac myocytes of high fat fed rats (322) and obese Zucker rats (91), blocking CD36-mediated fatty acid uptake in these insulin-resistant tissues (91, 322), using the specific CD36 inhibitor SSO, lowered the augmented rate of fatty acid esterification. A similar effect occurred when CD36 was ablated in a model of lipotoxic cardiac myopathy (480). This indicates that the upregulation of CD36 at the plasma membrane in insulin resistance is a key factor contributing to the accumulation of intracellular lipids.

A) TIME COURSE OF CD36 RELOCATION. As established in the heart and muscle, the permanent CD36 relocation to the plasma membrane is an early event in the development of insulin resistance and cardiomyopathy. Importantly, it has been observed that the increase in CD36-mediated fatty acid uptake in cardiac myocytes precedes changes in glucose uptake. Namely, in cardiac myocytes from insulin-resistant prediabetic obese Zucker rats, CD36 translocation and increased basal fatty acid uptake occurred in the absence of changes in basal and insulin-stimulated glucose uptake (92). Upon the transition from insulin resistance to type 2 diabetes, as seen in high fat fed rats (322) and diabetic Zucker rats (Luiken et al., unpublished results), CD36 translocation and increased basal fatty acid uptake are accompanied by concurrent decreases in basal and insulin-stimulated glucose uptake. Hence, during the development of diabetes there will be an increased juxtaposed localization of CD36 and GLUT4, with CD36 being at the cell surface and GLUT4 being localized intracellularly (Fig. 8). Moreover, it has been known for some time that the insulin-sensitive glucose transporter GLUT4 is retained within the intracellular $\operatorname{depot}(\mathrm{s})$ in obese skel- etal muscle in animals $(57,58)$ and in humans $(12,361)$. The strong inverse relationship $[r=-0.91$ (71) and $r=$ -0.94 (387)] between plasmalemmal GLUT4 and CD36 in insulin-resistant skeletal muscle in several studies is striking $(71,387)$ and contrasts with the healthy situation, in which CD36 and GLUT4 have been found each to be equally distributed between endosomes and the sarcolemma (see sect. Iv $A$ ). These combined findings suggest that in the healthy heart and muscle CD36 and GLUT4 are stored in separate subcompartments of the recycling endosomes and that these transporters are recruited via signaling and/or trafficking proteins specific for either one of these transporters. Then, upon development of insulin resistance, alterations in the signaling and/or trafficking proteins specifically dedicated to CD36 translocation could result in a selective and permanent CD36 translocation to the sarcolemma, without a change in subcellular distribution of GLUT4. Indeed, evidence for the existence of signaling and/or trafficking events that are specifically associated with CD36 has recently been reported when the small Ras-like GTPase rab 11 (see sect. $v B$ ) was observed to be attached to endosomal CD36 vesicles and not to GLUT4 vesicles (308).

Permanent CD36 relocation to the sarcolemma has also been shown to precede the loss of cardiac function, as 4 wk of high-fat feeding already resulted in increased cell surface localization of CD36 in the absence of a decrease in cardiac functional parameters, whereas a high-fat diet for 8 wk showed sustained surface presence of CD36 concomitant with decreased cardiac functioning (322). In combination with the causal relationship of increased sarcolemmal CD36 abundance and intramyocellular lipid accumulation, CD36 is beginning to be regarded as a key factor in the development of type 2 diabetes in heart and muscle.

B) CD36 AND FATTY ACID TRANSPORT CONTRIBUTE TO INTRAMUSCULAR LIPID ACCUMULATION, NOT REDUCED FATTY ACID OXIDATION. In view of a developing consensus that intramuscular lipids can accumulate in muscle despite concurrent increases in fatty acid oxidation, we have compared rates of CD36-mediated fatty acid transport with rates of fatty metabolism in muscles of obese Zucker rats (197). These studies have shown that plasmalemmal CD36 was again permanently relocated to the plasma membrane (197), as we had shown previously in this model (273). This plasmalemmal increase in CD36 in muscle of obese animals was highly correlated with an increased rate of fatty acid transport into muscle. Concomitantly, the rates of fatty acid esterification and oxidation were also both increased. However, in relation to the increased rate of fatty acid transport, the rate of esterification was eightfold greater than the increase in fatty acid oxidation (197). These studies strongly suggest that fatty acid transport into muscle cell is in excess of the capacity for them to be oxidized and, hence, lipids accumulate within the muscle 

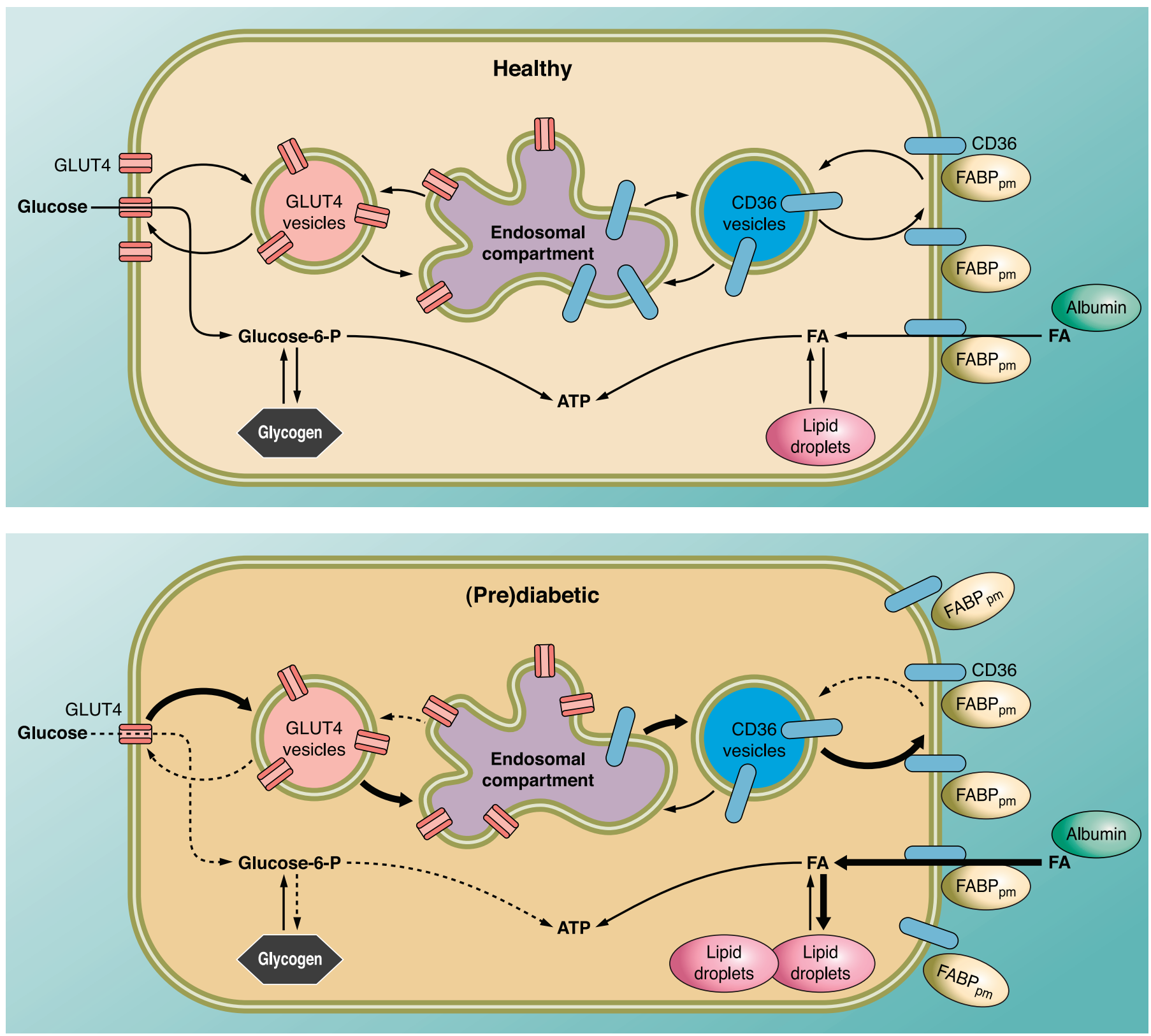

FIG. 8. Juxtaposition of CD36 and GLUT4 in insulin-resistant muscle. Schematic presentation showing the role of the substrate transporters CD36 and GLUT4 in myocytes under healthy conditions (top) and a hypothetical model for the development of an impaired GLUT4 translocation in (pre)diabetic state (bottom). While in healthy conditions, both CD36 and GLUT4 are about equally distributed between endosomes and the sarcolemma, in the (pre)diabetic state, there is a shift in CD36 localization from the endosomes to the sarcolemma resulting in enhanced fatty acid uptake, storage of fatty acids into triacylglyceroles (TAG), and subsequent inhibition of insulin signaling by fatty acid metabolites such as diacylglyceroles and ceramides. Thereafter, translocation of GLUT4 from endosomes to the sarcolemma is inhibited, resulting in lowered glucose uptake and decreased incorporation into glycogen. At that stage, the muscle has become insulin resistant. FA, (long-chain) fatty acid.

(197). Thus the CD36-mediated increase in fatty acid transport, rather than alterations in fatty acid oxidation, is the key factor contributing to the increase in intramuscular lipid accumulation in insulin-resistant muscle.

C) MECHANISMS RESPONSIBLE FOR PERMANENT CD36 RELOCATION TO THE SARCOLEMma. As detailed in sections Iv $A$ and $\mathrm{v}$, in the healthy heart and skeletal muscle, fatty acid uptake is increased by insulin or by contraction due to transloca- tion of CD36 from insulin-responsive PI3K/PKB/Akt-mediated endosomal stores or from contraction-responsive AMPK-mediated endosomal stores, respectively, to the sarcolemma. In the insulin-resistant heart, insulin was unable to further increase the already elevated basal fatty acid uptake rate, but activation of AMPK successfully further increased fatty acid uptake (91). In addition, insulin did not further increase the elevated sarcolemmal 
CD36 pool, nor did it further deplete CD36 from the endosomal stores $(91,322)$. These observations suggest that insulin-responsive PI3K/PKB/Akt-mediated endosomal stores are likely the source of the extra amount of CD36 present at the sarcolemma in the insulin-resistant heart. This, however, may not be the case in insulinresistant skeletal muscle, in which plasmalemmal CD36 is also permanently upregulated (164), because in this tissue both insulin and muscle contraction failed to induce a further increase in plasmalemmal CD36 (164). The fact that skeletal muscle becomes additionally contraction resistant, in contrast to heart, is a subtle difference between both tissues in the onset of insulin resistance. This is likely related to the fact that a safety system is built into the heart so that it still can increase fatty acid uptake upon increased contractile performance for the production of energy. Nonetheless, in both heart and skeletal muscle, permanent relocation of CD36 from insulin-responsive PI3K/PKB/Akt-mediated endosomal stores is likely a main contributor to the development of insulin resistance.

What then are the signals that permanently deplete the insulin-responsive endosomal stores from CD36? A clue is provided by the elevated basal phosphorylation of PKB/Akt and its distal target PRAS40 in hearts from high fat fed rats, whereas AMPK activity was not affected by the diet. Moreover, increased basal PKB/Akt activity together with impaired insulin-stimulated $\mathrm{PKB} /$ Akt activity have been reported in skeletal muscle from $o b / o b$ mice (301). Together these findings suggest that the elevated basal activity of $\mathrm{PKB} / \mathrm{Akt}$ may contribute to the sustained presence of CD36 at the sarcolemma in insulin-resistant heart and skeletal muscle, although other diet-induced changes, for instance, in the as yet undisclosed trafficking machinery regulating CD36 endocytosis and exocytosis (see sect. $\mathrm{v} B$ ), might also play a role.

In prediabetic Zucker rats, plasma insulin levels are sevenfold elevated and might be responsible for CD36 relocalization in heart and muscle $(91,164,273)$. In contrast, systemic hyperinsulinemia was not observed in high fat diet fed rats (321). Especially this latter observation may suggest that changes in the activity of other (insulinindependent) regulators of PKB/Akt phosphorylation, such as PI3K $\gamma$ - and $\beta 2$-adrenergic receptor signaling pathways, CaMK, protein phosphatase $2 \mathrm{~A}$, and the sympathetic nervous system (126), could perhaps be responsible for elevated PKB/Akt phosphorylation and subsequent permanent CD36 relocation to the sarcolemma in the insulin-resistant heart and skeletal muscle.

D) EMERging Picture of MEChanisms Involved. From the studies in muscles and hearts of high fat fed animals, obese Zucker rats, and ZDF rats, a picture emerges which suggests that increased rates of fatty acid transport are regulated by two mechanisms.

1) With moderate and modest insulin resistance (high fat feeding and obese Zucker rats), fatty acid trans- port is increased by the permanent relocation of CD36 from an intracellular depot to the plasma membrane without any alteration in the protein expression of CD36 (273, $322)$.

2) With more severe insulin resistance (male ZDF rats, high fat fed female $\mathrm{ZDF}$ rats), the total cellular pool of CD36 is increased $(71,387)$, which likely allows for a much greater increase in CD36 at the plasma membrane.

\section{Human studies}

There are only a few studies that have examined fatty acid transport and transporters in human obesity and type 2 diabetes. Understandably, this work is largely descriptive and has necessarily focused on the most accessible tissues, adipose tissue (not discussed here) and skeletal muscle.

It was initially reported that skeletal muscle fatty acid uptake in human muscle is reduced in type 2 diabetes $(35,476)$. However, these observations are likely incorrect, because these conclusions appear to be based on problematic, indirect measurements, and the data do not concur with more recent direct determination of fatty acid transport rates across the plasma membrane in muscles of diabetic animals $(71,387)$ or humans with type 2 diabetes (48).

Direct determination of skeletal muscle fatty acid transport, using giant sarcolemmal vesicles, has shown that rates of fatty acid transport are markedly increased in skeletal muscle of obese individuals and those with type 2 diabetes (48). In contrast, just as in some animal models of insulin resistance (see above), skeletal muscle fatty acid transporters (FATP1 mRNA and CD36 mRNA and protein) were not altered in human obesity and type 2 diabetes $(34,48,330)$, or were altered inconsistently $\left(\mathrm{FABP}_{\mathrm{pm}}\right.$ protein; Refs. $\left.48,330,384\right)$. Instead, the increase in the fatty acid transport rate into obese and type 2 diabetic muscle was associated with an increase in the plasmalemmal content of CD36 (obesity, +40\%; type 2 diabetes, $+50-76 \%$; Refs. 18, 48) and FATP4 (type 2 diabetes, 20\%; Ref. 18), whereas plasmalemmal $\mathrm{FABP}_{\mathrm{pm}}$ and FATP1 were not altered $(18,48)$. Indeed, the increase in plasmalemmal CD36 in lean, overweight, and obese individuals and type 2 diabetes patients correlated well with their increased rates of fatty acid transport $(r=$ 0.93), an index of insulin resistance (48). Moreover, the rates of fatty acid transport were also positively associated with the intramuscular triacylglycerol concentrations ( $r=0.93$ ) (48) (Fig. 9). Notably therefore, in human obesity and type 2 diabetes, just as in animal models of insulin resistance $(164,197,273)$, skeletal muscle fatty acid transport into muscle and intramuscular triacylglycerol accumulation are increased largely in association with the permanent relocation of CD36 to the plasma 
membrane (48), as well as possibly a small increase in the plasmalemmal FATP4 pool (18).

\section{Fiber type-specific and gender differences}

A) FIBER TYPE. Skeletal muscles consist of red (oxidative) and white (glycolytic) fibers. Compared with white muscles, the red muscles have a higher capacity for fatty acid oxidation and esterification $(42,114,164)$ and insulin-stimulated glucose transport $(49,165,184,298,391)$. These differences are related, in part, to inherent differences (red $>$ white muscle) in their expression of PGC$1 \alpha$, their glucose and fatty acid transport proteins, and their enzymes for metabolizing fatty acids $(23,45,184$, 298). In view of these differences, the potential dysregulation in these processes may be greater in red muscle. Indeed, oxidative (red) muscles exhibit a 2.5-fold greater impairment in insulin-stimulated glucose transport than glycolytic (white) muscle in response to a high-fat diet (474), and ZDF rats exhibit a reduction only in red muscle GLUT4 (-40\%) but not in white muscle GLUT4 (128). In human obesity and type 2 diabetes, excess intramuscular lipid accumulation ( +20 to $+56 \%$ ) occurs in red (I and IIa) but not in white (IIb) muscle fibers (180). There is some indication that the greater lipid accumulation in red muscles is related to muscle-specific upregulation of fatty acid transporters and transport in red muscle compared with white muscle (see below) (Fig. 10). Such fiber-specific differences could account for the accumulation of red muscle intramuscular lipids that can interfere with insulin signaling.

An increased fatty acid disposal has been observed in red but not in white muscles in the high-fat diet-induced

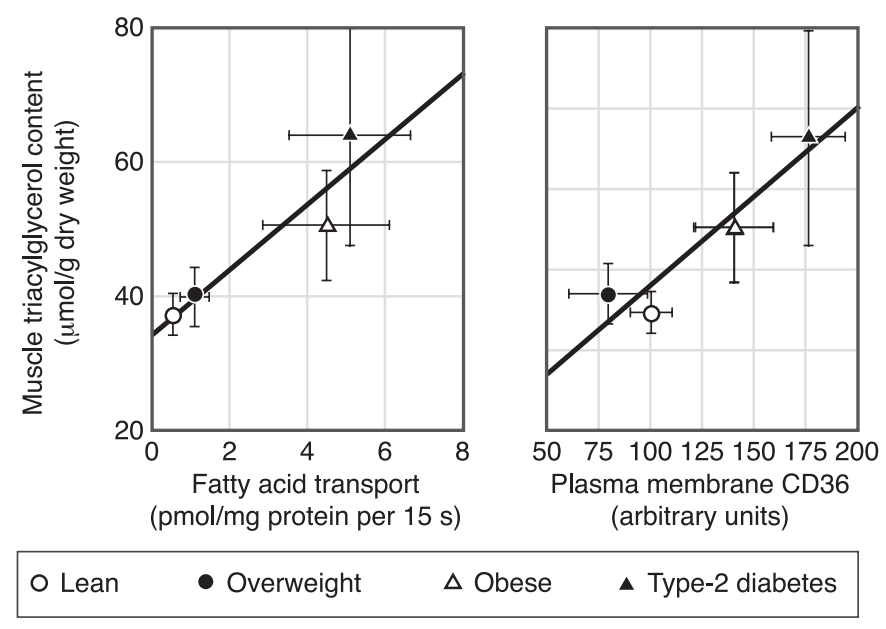

FIG. 9. Triacylglycerol accumulation in human obesity and type 2 diabetes is associated with increased rates of muscle fatty acid transport and increased plasmalemmal CD36 content. Fatty acid uptake rates and CD36 were determined in giant sarcolemmal vesicles prepared from rectus abdominus muscle biopsies obtained from lean [body mass index $(\mathrm{BMI})<25$ ], overweight $(\mathrm{BMI}=25-30)$, and obese $(\mathrm{BMI}>30)$ individuals and type 2 diabetics. [Redrawn from Bonen et al. (48).]

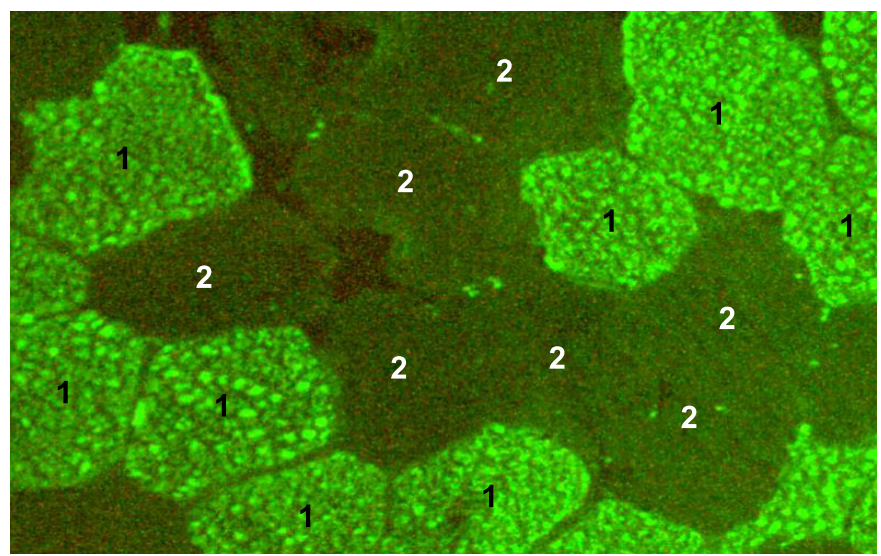

FIG. 10. Distribution and immunolocalization of CD36 in human skeletal muscle. A cryosection of human $\mathrm{m}$. vastus lateralis was stained for CD36, first by incubation with a specific anti-CD36 antibody, which was followed by incubation with a fluorescently labeled secondary antibody. Type 1 fibers were identified using antibodies specific for slow myosin heavy chain (data not shown). CD36 is more abundant in (slow oxidative) type 1 muscle fibers (indicated by 1 ) than in (fast glycolytic) type 2 fibers (2). At the subcellular level, CD36 immunostaining is seen both at the sarcolemma and in the cytoplasm (punctate staining pattern). [Figure courtesy of Dr. H. A. Keizer (236).]

model of insulin resistance, and this was associated with an increase in red muscle CD36 mRNA (182). Similarly, in red, but not in white muscle, fasting induced an increase in plasmalemmal $\mathrm{FABP}_{\mathrm{pm}}$ (444). In obese Zucker rats, fatty acid transport (197) and incorporation into triacylglycerol depots $(197,446)$ are increased more in red muscle than in white muscle. These increased rates of fatty acid transport into red muscle were twofold greater than into white obese muscle, and these differences were associated with a concomitantly greater concentration in plasmalemmal CD36 in red (increase of 100 arbitrary units) than in white muscle (increase of 25 arbitrary units) (197). Other fatty acid transporters located at the plasma membrane $\left(\mathrm{FABP}_{\mathrm{pm}}, \mathrm{FATP} 1\right.$ and -4$)$ were not altered in either red or white muscles of obese rats $(164,197)$, except for one report $(+61 \%$ in red muscle plasmalemmal $\mathrm{FABP}_{\mathrm{pm}}$ ) (446). Along similar lines, in the transition from insulin resistance to type 2 diabetes in ZDF rats, there was an increase in red muscle, but not white muscle, fatty acid transport rates due to concomitant increases in CD36 protein expression and plasmalemmal content, while no changes occurred in either red or white muscle plasmalemmal $\mathrm{FABP}_{\mathrm{pm}}$ (71). Moreover, the increase in red muscle plasmalemmal CD36 was inversely correlated with the plasmalemmal GLUT4 $(r=-0.90)$ (71).

Taken together, the available evidence indicates that red muscle exhibits a greater propensity for developing insulin resistance. This appears to be related, in part, to changes in intramuscular lipid accumulation in red compared with white muscle, likely as a result of changes in the expression and/or plasmalemmal content of CD36, which facilitates a greater rate of fatty acid uptake. 
B) GENDER DIFFERENCES. It appears that there are gender differences in insulin resistance and in the responses of fatty acid transporters to an altered fatty acid milieu and insulin. Specifically, in male rats, an acute (2 h), fourfold increase in circulating fatty acids impaired insulin signaling (IRS-1 phosphorylation, -30\%; IRS-1-associated PI3K activity, $-48 \%$ ) and induced a $40 \%$ reduction in the skeletal muscle insulin-stimulated glucose disposal rate. This was accompanied by a $50 \%$ reduction in skeletal muscle CD36 protein expression (189). Surprisingly, none of these changes was observed in female rats (189).

Considerable differences in FATP1 mRNA have also been observed among men and women. FATP1 mRNA expression in skeletal muscle is $\sim 3.7$-fold greater in lean women than in lean men (34), and insulin infusion (3 h) reduced FATP1 mRNA in lean women but not lean men, nor in obese nondiabetic and diabetic men and women (34). In addition, in women, but not in men, BMI and FATP1 mRNA were inversely related $(r=-0.74)$ (34). In the absence of any measures of fatty acid transport and in the FATP proteins in these studies, it is difficult to determine the pathophysiological significance of these observations.

Clearly, the few available studies in both animals and humans do begin to suggest that there may be gender differences with respect to insulin resistance and fatty acid metabolism, including fatty acid transport and transporters. This warrants further investigation.

\section{Insulin resistance in spontaneously hypertensive rats is not attributable to CD36 ablation}

A series of genetic studies combining the use of cDNA microarrays, congenic mapping, and radiation hybrid mapping in spontaneously hypertensive rats (SHR), a rat model of insulin resistance and hypertension, suggested that CD36 deficiency is at the peak of linkage to the SHR alterations in fatty acid and glucose metabolism (7, $89,337,338$ ). The authors concluded that CD36 deficiency provides a plausible basis for the development of insulin resistance through a primary defect in cellular fatty acid transport. Support for this notion was provided by the observation that transgenic rescue of CD36 improved insulin sensitivity (337). We fully agree with the notion that CD36 is involved in insulin resistance, but we envisage that an upregulation rather than a downregulation of CD36 is causal to insulin resistance. In fact, a possible downregulation of CD36 seems counterintuitive with regard to the accumulation of fatty acids and their metabolites in peripheral tissues. Remarkably, in contrast to the North American SHR strain used in the aforementioned studies, an SHR strain in Japan did express CD36 (146), uncoupling CD36 from the development of insulin resistance in this rodent model. The supporting evidence linking an apparent null expression of CD36 in North Ameri- can SHR with insulin resistance are inconsistent. For example, transgenic expression of wild-type CD36 in the North American SHR strain increased glucose disposal (OGTT) and muscle glycogenesis and reduced circulating fatty acids (337). Yet, in subsequent work with these CD36 transgenic SHR, neither glucose disposal (OGTT) nor muscle glucose oxidation was improved (340). In strokeprone SHR animals, lipid metabolism differed, despite similar expression of adipocyte CD36 protein (89). Moreover, other studies suggest that CD36 ablation improves insulin sensitivity $(123,147)$, and an increase in CD36 is linked strongly with insulin resistance $(48,91,273)$, as this leads to an accumulation of intramuscular fatty acid products that can interfere with insulin signaling. From more recent work it now appears that in fact North American SHR are not null for CD36 (43). This protein is expressed in many tissues of these animals albeit at a reduced level in heart $(-26 \%)$, red $(-40 \%)$ and white muscles $(-53 \%)$, liver $(-75 \%)$, and adipose tissue $(-46 \%)$ (43). Thus it is very difficult to support the proposition that the North American SHR strain fails to express CD36 protein and that a CD36 deficiency underlies insulin resistance in these animals.

\section{Type 1 Diabetes}

Lipid metabolism is also altered in type 1 diabetes, an effect that has been associated with altered fatty acid transport and transporters. For example, early studies indicated that heart and skeletal muscle CD36 protein expression was increased in streptozotocin (STZ)-induced diabetes, an animal model of type 1 diabetes (150, 329), suggesting an important role for this protein in taking up the increased circulating fatty acids in this model. Indeed, the intramuscular fatty acids are increased by $\sim 25 \%$ in STZ-induced diabetic rats (312). Detailed studies of moderate and severe STZ-induced diabetes have revealed tissue-specific responses in fatty acid transport and transporters (272). The relative changes and the magnitude of changes in fatty acid transport and transporters differed among the tissues examined and/or depended on the severity of diabetes. For example, fatty acid transport increased progressively with the severity of diabetes only in the heart (moderate, $+71 \%$; severe, $+143 \%)$. In skeletal muscle, fatty acid transport was upregulated to a similar extent in moderate $(+37 \%)$ and severe diabetes $(+28 \%)$. In contrast, in adipose tissue, fatty acid transport was not changed with moderate diabetes but upregulated $(+171 \%)$ in severe diabetes $(272)$. Concurrent with these changes in fatty acid transport, there were also concomitant changes in the protein expression and plasmalemmal content of CD36 (muscle and heart) and $\mathrm{FABP}_{\mathrm{pm}}$ (severely diabetic heart only), while in adipose tissue the CD36 plasmalemmal content, but not 
its protein expression, was increased in severe diabetes. These changes in protein expression were not consistently related to changes in the mRNAs of these transporters. However, it could be shown that the changes in the rate of fatty acid transport in these different tissues was related to the presence of both CD36 and $\mathrm{FABP}_{\mathrm{pm}}$ at the plasma membrane (272). Collectively, these studies indicate that changes in CD36 and $\mathrm{FABP}_{\mathrm{pm}}$ occur in animal models of type 1 diabetes, but the extent to which these changes contribute to altered fatty acid metabolism in the STZ-induced diabetic animal model remains to be determined.

\section{CONCLUSIONS AND PERSPECTIVES}

Membrane fatty acid transporters have emerged as a group of proteins serving a pivotal role in whole body lipid metabolism. By facilitating the transmembrane transport of fatty acids, these proteins dramatically accelerate fatty acid transport into cells (and their release from adipocytes). In addition, cellular entry of fatty acids appears to be regulated acutely by changes in plasmalemmal fatty acid transporter content, which occurs in response to selected stimuli (e.g., muscle contraction, insulin) and involves the translocation of specific fatty acid transporters from an intracellular storage compartment to the plasmalemma. Fatty acid transporters thus form an integral part of a complex network of plasma and cellular proteins that enable a rapid availability and unrestricted use of fatty acids in the cell yet keep them from exerting potential harmful effects related to their amphiphilic ("soaplike") characteristics. Finally, the occurrence of various types of fatty acid transporters with each displaying a characteristic pattern of tissue distribution further illustrates their role in cellular lipid homeostasis tuned to the metabolic requirements of a specific tissue. While the focus of this review was on heart and skeletal muscle, the concepts outlined for these tissues generally will presumably apply to all tissues with an active fatty acid metabolism.

\section{A. Integration of Regulatory Steps}

The new insights on the functioning of fatty acid transporters have further underscored the striking similarity between the regulation of the cellular utilization of fatty acids and that of glucose. This similarity relates to 1) the occurrence of a relatively small number of distinct proteins facilitating transmembrane substrate transport, 2) a tissue-specific expression pattern of these proteins, and 3) acute translocation of selected proteins to the plasma membrane in response to physiological stimuli, notably muscle contraction and insulin, to regulate cellular substrate uptake. It appears that nature has generated quite similar systems to safeguard a properly regulated cellular entry of two main substrates, long-chain fatty acids and glucose. A major corollary of these findings is that cellular fatty acid utilization, just like that of glucose, is regulated at three levels, i.e., 1) substrate delivery, 2) cellular uptake, and 3) intracellular metabolism (oxidation, storage, and so on, including substrate competition at the level of mitochondria).

Fatty acid transporters, just like GLUTs, function as a link among various metabolic regulatory steps aimed at adjusting cellular substrate metabolism and referred to as "metabolic coordination." For instance, muscle contraction, through activation of AMP kinase, increases the translocation of fatty acid transporters (CD36) to the sarcolemma to increase fatty acid uptake, and concomitantly increases mitochondrial fatty acid oxidation to produce ATP needed to sustain contraction. As a result, proper functioning of fatty acid transporters is of central importance to maintain homeostasis, and derangements therein likely will result in pathology (see below).

\section{Functioning of fatty acid transporters}

The molecular mechanism explaining the functioning of fatty acid transporters is not yet clear. Specifically, it is not known 1) whether fatty acid transporters function alone or as a (heteromeric) complex (e.g., CD36 and $\mathrm{FABP}_{\mathrm{pm}}$ ), 2) whether they act as transmembrane transporters or 3) merely function as docking site or cell surface receptor for fatty acids, whereafter the fatty acids cross the plasma membrane by simple diffusion, 4) whether the functioning of fatty acid transporters is dependent on or influenced by accessory proteins or by the lipid microenvironment, and finally 5) whether posttranslational modifications (palmitoylation, phosphorylation) would further influence their functioning. Importantly, the mode of action of fatty acid transporters may also differ among the key tissues involved in fatty acid handling. In this respect, it has been shown that the protein expression of each of the fatty acid transporters $\left(\right.$ FABP $_{\mathrm{pm}}, \mathrm{CD} 36, \mathrm{FATP} 4$, but not FATP1) is graded among muscle tissues in relation to their well-known differences in fatty acid utilization, i.e., heart $>>$ red muscle $>$ white muscle (45, 197, 286, 314, 329, 459). However, absolute quantities have been assessed only for CD36 (329) so that a quantitative comparison among fatty acid transporters is not yet possible.

Further insight into the molecular functioning of fatty acid transporters could also be obtained from their three-dimensional protein structures. However, unfortunately, a detailed structure is not yet known for any of the fatty acid transporters (cf. Fig. 1, $B$ and $C$ ). Although computer predictions of the protein structure based on the primary amino acid sequence have been presented for CD36 (156) and FATP (107), and some protein characteristics are known for $\mathrm{FABP}_{\mathrm{pm}}$ (419), these models must be interpreted with great caution as they may not represent the real structure. 
It has been argued that each of the fatty acid transporters also is known to support other metabolic functions, e.g., mitochondrial AspAT $\left(\mathrm{FABP}_{\mathrm{pm}}\right)$, multifunctional ligand receptor (CD36), and (very-long-chain) acylCoA synthetase (FATPs) (see sect. III). These findings have been interpreted to suggest that their functioning in cellular fatty acid uptake is artifactual. However, it should be noted that assigning dual functions to specific proteins is not uncommon. For instance, $\beta$-catenin functions in both cellular adhesion and transcriptional regulation (53), and integrins function both in cell adhesion and in cellular signaling (303).

\section{Is protein-mediated fatty acid transport rate-limiting in fatty acid utilization?}

In heart and muscle, glucose uptake mediated by the glucose transporter GLUT4, except during exercise (130), is considered the rate-limiting step in cellular glucose utilization (for review, see Refs. 207, 365, 428). Similarly, but perhaps not during exercise (242), it is also possible that protein-mediated fatty acid transport across the sarcolemma is rate-limiting in cardiac and muscular fatty acid utilization. Several lines of evidence do support this view.

First, the intracellular fatty acid concentration remains low at saturating fatty acid concentrations (see sect. $\mathrm{I} B$ ). This observation does not only indicate that the saturation kinetics of cellular fatty acid uptake reflects saturation of sarcolemmal transport rather than saturation of metabolism (287), but also supports the notion that fatty acid uptake may be a rate-limiting step in cardiac and muscular fatty acid utilization (285). Second, the fatty acid uptake kinetics in the presence (cardiac myocytes) and absence of metabolism (giant sarcolemmal vesicles) display a similar apparent $K_{\mathrm{m}}$ for fatty acid uptake $(285,286)$. Third, a recent study (283) showed that CPT-I, which converts fatty acyl-CoA into fatty acyl-Lcarnitine and is generally thought to be the rate-limiting step in cardiac fatty acid utilization (for review, see Ref. 296), cannot be the only parameter governing the rate of fatty acid utilization. Specifically, a chronic partial $(\sim 50 \%)$ inhibition of CPT-I by administration of etomoxir to rats did not affect fatty acid uptake and metabolism by cardiac myocytes (283), strongly arguing against CPT-I as the primary site determining cardiac fatty acid fluxes. As a result, other sites in cardiac fatty acid utilization, most notably sarcolemmal protein-mediated fatty acid transport, may also function in regulating cardiac fatty acid flux. The mechanism of such regulation may differ among the various fatty acid transporters but will include intracellular translocation of fatty acid transporters to the plasma membrane (see sect. $\mathrm{r} A$ ) and to mitochondria (see sect. IVC), and may also comprise modulation of their involvement by, for instance, changes in the interaction with accessory proteins.

\section{B. Fatty Acid Transporters as Potential Therapeutic Targets}

The role of fatty acid transporters in the control of cellular fatty acid uptake under normal conditions and their implication in metabolic alterations occurring in certain disease conditions suggest that fatty acid transporters are an attractive therapeutic target to manipulate cellular fatty acid utilization. This would apply especially to obesity and insulin resistance whereby increased rates of fatty acid transport are a key factor contributing to the intracellular lipid accumulation in heart and muscle tissue, which in turn interferes with the insulin signal transduction cascade required to induce GLUT4 translocation (see sect. VII $B$ ). Indeed, in studies in which CD36 has been ablated (147) or in which the increase in plasmalemmal CD36 content has been prevented (387), there is clear evidence of an improved insulin sensitivity (i.e., blunting the progression of high-fat diet-induced insulin resistance). In addition, CD36 ablation was found to rescue myocardial lipotoxicity and myocardial dysfunction induced by PPAR $\alpha$ overexpression (480). To date, there are as yet no effective pharmacological agents that specifically inhibit fatty acid transport. Unfortunately, the CD36 inhibitor SSO is unsuitable for use in vivo due to its limited stability in solution. Nevertheless, development of inhibitors that limit fatty acid uptake would represent a novel therapeutic strategy against insulin resistance.

In a broader perspective, manipulation of cellular substrate uptake by modulating the presence or functioning of specific substrate transporters in the plasma membrane has been suggested as a means to alter cellular substrate preference (140). Such an approach is expected to be of interest not only for heart (140) but also for skeletal muscle, as intermediary metabolism is regulated similarly in these organs while both are sensitive to a chronic change in the balance of glucose and fatty acid utilization. Theoretically, modulating the cellular distribution of, in particular, the substrate transporters GLUT4 and CD36 in the diseased heart or muscle may normalize substrate metabolism and allow them to function normally. In practice this would require the selective recruitment of either GLUT4 or CD36 to the membrane, or their selective internalization, while so far it was found that these transporters respond to physiological stimuli (contraction, insulin) by their simultaneous recruitment (see sect. IvA). However, proof of concept for such selectivity comes from studies in rat cardiomyocytes indicating that arsenite (282) and dipyridamole (274) specifically recruit GLUT4 and CD36, respectively, leading to selectively increased glucose and fatty acid utilization, respectively. 
Although the intracellular targets of these pharmacological compounds are not known, most likely they activate or inhibit trafficking proteins specifically involved in either GLUT4 or CD36 translocation (376). As a result, these recent findings illustrate the feasibility of the general concept that selective transporter translocation can be applied to alter substrate preference and thus holds promise as a target for so-called metabolic modulation therapy (422).

\section{ACKNOWLEDGMENTS}

We thank Dr. Robert W. Schwenk for assistance with the illustrations.

Addresses for reprint requests and other correspondence: J. F. C. Glatz and J. J. F. P. Luiken, Dept. of Molecular Genetics, Maastricht University, PO Box 616, NL-6200 MD Maastricht, The Netherlands (e-mail: glatz@gen.unimaas.nl; j.luiken@gen.unimaas.nl); A. Bonen, Dept. of Human Health and Nutritional Sciences, University of Guelph, Guelph, Ontario Canada, N1G 2W1 (e-mail: abonen @uoguelph. ca).

\section{GRANTS}

Studies in the authors' laboratories were supported by the Netherlands Organization for Health Research and Development (NWO-ZonMw Grant 912-04-075), the European Commission (Integrated Project LSHM-CT-2004-005272, Exgenesis), the Natural Sciences and Engineering Research Council of Canada, the Canadian Foundation for Innovation, the Heart and Stroke Foundation of Ontario, the Canadian Institutes of Health Research, and the Canada Research Chair program.

J. F. C. Glatz is Netherlands Heart Foundation Professor of Cardiac Metabolism.

J. J. F. P. Luiken was the recipient of a VIDI-Innovational Research Award from the Netherlands Organization for Scientific Research. Health.

A. Bonen is the Canada Research Chair in Metabolism and

\section{REFERENCES}

1. Abel ED, Litwin SE, Sweeney G. Cardiac remodeling in obesity. Physiol Rev 88: 389-419, 2008.

2. Abumrad N, Coburn C, Ibrahimi A. Membrane proteins implicated in long-chain fatty acid uptake by mammalian cells: CD36, FATP and FABPm. Biochim Biophys Acta 1441: 4-13, 1999.

3. Abumrad N, Harmon C, Ibrahimi A. Membrane transport of long-chain fatty acids: evidence for a facilitated process. $J$ Lipid Res 39: 2309-2318, 1998.

4. Abumrad NA, El-Maghrabi MR, Amri EZ, Lopez E, Grimaldi P. Cloning of a rat adipocyte membrane protein implicated in binding or transport of long chain fatty acids that is induced during preadipocyte differentiation. Homology with human CD36. J Biol Chem 268: 17665-17668, 1993.

5. Abumrad NA, Park JH, Park CR. Permeation of long-chain fatty acid into adipocytes. Kinetics, specificity, and evidence for involvement of a membrane protein. J Biol Chem 259: 8945-8953, 1984.

6. Abumrad NA, Perkins RC, Park JH, Park CR. Mechanism of long chain fatty acid permeation in the isolated adipocyte. J Biol Chem 256: 9183-9191, 1981.

7. Aitman TJ, Glazier AM, Wallace CA, Cooper LD, Norsworthy PJ, Wahid FN, Al-Majali KM, Trembling PM, Mann CJ, Shoulders CC, Garf D, St. Lezin E, Kurtz TW, Kren V, Pravenec M,
Ibrahimi A, Abumrad NA, Stanton LW, Scott J. Identification of Cd36 (Fat) as an insulin-resistance gene causing defective fatty acid and glucose metabolism in hypertensive rats. Nature Genet 21: 76-83, 1999.

8. Aledo JC, Lavoie L, Volchuk A, Keller SR, Klip A, Hundal HS. Identification and characterization of two distinct intracellular GLUT4 pools in rat skeletal muscle: evidence for an endosomal and an insulin-sensitive GLUT4 compartment. Biochem J 325: 727-732, 1997.

9. Alessi DR, James SR, Downes CP, Holmes AB, Gaffney PR, Reese CB, Cohen P. Characterization of a 3-phosphoinositidedependent protein kinase which phosphorylates and activates protein kinase Balpha. Curr Biol 7: 261-269, 1997.

10. Allard MF. Energy substrate metabolism in cardiac hypertrophy. Curr Hypertens Rep 6: 430-435, 2004.

11. Andersen CB, Roth RA, Conti M. Protein kinase B/Akt induces resumption of meiosis in Xenopus oocytes. J Biol Chem 273: 18705-18708, 1998.

12. Andersen PH, Lund S, Vestergaard H, Junker S, Kahn BB, Pedersen O. Expression of the major insulin regulatable glucose transporter (GLUT4) in skeletal muscle of noninsulin-dependent diabetic patients and healthy subjects before and after insulin infusion. J Clin Endocrinol Metab 77: 27-32, 1993.

13. Asch AS, Liu I, Briccetti FM, Barnwell JW, Kwakye-Berko F, Dokun A, Goldberger J, Pernambuco M. Analysis of CD36 binding domains: ligand specificity controlled by dephosphorylation of an ectodomain. Science 262: 1436-1440, 1993.

14. Augustus AS, Buchanan J, Addya S, Rengo G, Pestell RG, Fortina P, Koch WJ, Bensadoun A, Abel ED, Lisanti MP. Substrate uptake and metabolism are preserved in hypertrophic caveolin-3 knockout hearts. Am J Physiol Heart Circ Physiol 295: H657-H666, 2008.

15. Augustus AS, Buchanan J, Gutman E, Rengo G, Pestell RG, Fortina P, Koch WJ, Bensadoun A, Abel ED, Lisanti MP. Hearts lacking caveolin-1 develop hypertrophy with normal cardiac substrate metabolism. Cell Cycle 7: 2509-2518, 2008.

16. Baillie AG, Coburn CT, Abumrad NA. Reversible binding of long-chain fatty acids to purified FAT, the adipose CD36 homolog. J Membr Biol 153: 75-81, 1996.

17. Bandyopadhyay G, Standaert ML, Galloway L, Moscat J, Farese RV. Evidence for involvement of protein kinase C (PKC)-zeta and noninvolvement of diacylglycerol-sensitive PKCs in insulinstimulated glucose transport in L6 myotubes. Endocrinology 138: 4721-4731, 1997.

18. Bandyopadhyay GK, Yu JG, Ofrecio J, Olefsky JM. Increased malonyl-CoA levels in muscle from obese and type 2 diabetic subjects lead to decreased fatty acid oxidation and increased lipogenesis; thiazolidinedione treatment reverses these defects. Diabetes 55: 2277-2285, 2006.

19. Barger PM, Kelly DP. PPAR signaling in the control of cardiac energy metabolism. Trends Cardiovasc Med 10: 238-245, 2000.

20. Bastie CC, Hajri T, Drover VA, Grimaldi PA, Abumrad NA. CD36 in myocytes channels fatty acids to a lipase-accessible triglyceride pool that is related to cell lipid and insulin responsiveness. Diabetes 53: 2209-2216, 2004.

21. Benton CR, Campbell SE, Tonouchi M, Hatta H, Bonen A. Monocarboxylate transporters in subsarcolemmal and intermyofibrillar mitochondria. Biochem Biophys Res Commun 323: 249-253, 2004.

22. Benton CR, Koonen DP, Calles-Escandon J, Tandon NN, Glatz JFC, Luiken JJFP, Heikkila JJ, Bonen A. Differential effects of muscle contraction and PPAR agonists on the expression of fatty acid transporters in rat skeletal muscle. J Physiol 573: 199-210, 2006.

23. Benton CR, Nickerson J, Lally J, Han XX, Holloway GP, Glatz JFC, Luiken JJFP, Graham TE, Heikkila JJ, Bonen A. Modest PGC-1 $\alpha$ overexpression in muscle in vivo is sufficient to increase insulin sensitivity and palmitate oxidation in SS, not IMF, mitochondria. J Biol Chem 283: 4228-4240, 2008.

24. Bergemann C, Loken C, Becker C, Graf B, Hamidizadeh M, Fischer Y. Inhibition of glucose transport by cyclic GMP in cardiomyocytes. Life Sci 69: 1391-1406, 2001. 
25. Berger M, Schmidt MF. Characterization of a protein fatty acylesterase present in microsomal membranes of diverse origin. $J$ Biol Chem 261: 14912-14918, 1986.

26. Berk PD, Wada H, Horio Y, Potter BJ, Sorrentino D, Zhou SL, Isola LM, Stump D, Kiang CL, Thung S. Plasma membrane fatty acid-binding protein and mitochondrial glutamic-oxaloacetic transaminase of rat liver are related. Proc Natl Acad Sci USA 87: 3484-3488, 1990

27. Berk PD, Zhou SL, Kiang CL, Stump D, Bradbury M, Isola L. Uptake of long chain fatty acids is selectively up-regulated in adipocytes of Zucker rats with genetic obesity and non-insulindependent diabetes mellitus. J Biol Chem 272: 8830-8835, 1997.

28. Berk PD, Zhou SL, Kiang CL, Stump D, Fan X, Bradbury M. Selective upregulation of fatty acid uptake by adipocytes characterizes both genetic and diet-induced obesity in rodents. $J$ Biol Chem 274: 28626-28631, 1999.

29. Bertrand L, Horman S, Beauloye C, Vanoverschelde JL. Insulin signalling in the heart. Cardiovasc Res 79: 238-248, 2008.

30. Bezaire V, Bruce CR, Heigenhauser GJ, Tandon NN, Glatz JF, Luiken JJ, Bonen A, Spriet LL. Identification of fatty acid translocase on human skeletal muscle mitochondrial membranes: essential role in fatty acid oxidation. Am J Physiol Endocrinol Metab 290: E509-E515, 2006.

31. Bezaire V, Heigenhauser GJ, Spriet LL. Regulation of CPT I activity in intermyofibrillar and subsarcolemmal mitochondria from human and rat skeletal muscle. Am J Physiol Endocrinol Metab 286: E85-E91, 2004

32. Binas B, Danneberg H, McWhir J, Mullins L, Clark AJ. Requirement for the heart-type fatty acid binding protein in cardiac fatty acid utilization. FASEB $J$ 13: 805-812, 1999.

33. Binas B, Han XX, Eroll E, Luiken JJFP, Glatz JF, Dyck DJ, Motazavi R, Adihetty PJ, Hood DA, Bonen A. A null mutation in H-FABP only partially inhibits skeletal muscle fatty acid metabolism. Am J Physiol Endocrinol Metab 285: E481-E489, 2003.

34. Binnert C, Koistinen HA, Martin G, Andreelli F, Ebeling P, Koivisto VA, Laville M, Auwerx J, Vidal H. Fatty acid transport protein-1 mRNA expression in skeletal muscle and in adipose tissue in humans. Am J Physiol Endocrinol Metab 279: E1072E1079, 2000 .

35. Blaak EE, Wagenmakers AJ. The fate of $\left[\mathrm{U}_{-}{ }^{13} \mathrm{C}\right]$ palmitate extracted by skeletal muscle in subjects with type 2 diabetes and control subjects. Diabetes 51: 784-789, 2002.

36. Boden G. Free fatty acids, insulin resistance, and type 2 diabetes mellitus. Proc Asoc Am Physicians 111: 241-248, 1999.

37. Boden G, Chen X, Iqbal N. Acute lowering of plasma fatty acids lowers basal insulin secretion in diabetic and nondiabetic subjects. Diabetes 47: 1609-1612, 1998.

38. Boden G, Jadali F, White J, Liang Y, Mozzoli M, Chen X, Coleman E, Smith C. Effects of fat on insulin-stimulated carbohydrate metabolism in normal men. J Clin Invest 88: 960-966, 1991.

39. Bonen A, Chabowski A, Luiken JJFP, Glatz JFC. Is membrane transport of FFA mediated by lipid, protein, or both? Mechanisms and regulation of protein-mediated cellular fatty acid uptake: molecular, biochemical, and physiological evidence. Physiology 22: 15-29, 2007.

40. Bonen A, Dohm GL, van Loon LJ. Lipid metabolism, exercise and insulin action. Essays Biochem 42: 47-59, 2006.

41. Bonen A, Dyck DJ, Ibrahimi A, Abumrad NA. Muscle contractile activity increases fatty acid metabolism and transport and FAT/CD36. Am J Physiol Endocrinol Metab 276: E642-E649, 1999.

42. Bonen A, Han XX, Habets DDJ, Febbraio M, Glatz JFC, Luiken JJFP. A null mutation in skeletal muscle FAT/CD36 reveals its essential role in insulin- and AICAR-stimulated fatty acid metabolism. Am J Physiol Endocrinol Metab 292: E1740-E1749, 2007.

43. Bonen A, Han XX, Tandon NN, Glatz JFC, Lally J, Snook L, Luiken JJFP. FAT/CD36 expression is not ablated in spontaneously hypertensive rats. J Lipid Res 50: 740-748, 2009.

44. Bonen A, Luiken JJFP, Arumugam Y, Glatz JFC, Tandon NN. Acute regulation of fatty acid uptake involves the cellular redistribution of fatty acid translocase. J Biol Chem 275: 14501-14508, 2000 .
45. Bonen A, Luiken JJFP, Lui S, Dyck DJ, Kiens B, Kristiansen S, Turcotte L, van der Vusse GJ, Glatz JFC. Palmitate transport and fatty acid transporters in red and white muscles. Am J Physiol Endocrinol Metab 275: E471-E478, 1998.

46. Bonen A, Miskovic D, Kiens B. Fatty acid transporters (FABPpm, FAT, FATP) in human muscle. Can J Appl Physiol 24: 515-523, 1999 .

47. Bonen A, Nickerson JG, Momken I, Chabowski A, CallesEscandon J, Tandon NN, Glatz JF, Luiken JJ. Tissue-specific and fatty acid transporter-specific changes in heart and soleus muscle over a 1-yr period. Mol Cell Biochem 291: 145-154, 2006.

48. Bonen A, Parolin ML, Steinberg GR, Calles-Escandon J, Tandon NN, Glatz JFC, Luiken JJFP, Heigenhauser GJF, Dyck DJ. Triacylglycerol accumulation in human obesity and type 2 diabetes is associated with increased rates of skeletal muscle fatty acid transport and increased sarcolemmal FAT/CD36. FASEB $J$ 18: 1144-1146, 2004.

49. Bonen A, Tan MH, Watson-Wright WM. Insulin binding and glucose uptake differences in rodent skeletal muscles. Diabetes 30: 702-704, 1981.

50. Boushel R, Gnaiger E, Schjerling P, Skovbro M, Kraunsoe R, Dela F. Patients with type 2 diabetes have normal mitochondrial function in skeletal muscle. Diabetologia 50: 790-796, 2007.

51. Bradbury MW, Berk PD. Mitochondrial aspartate aminotransferase: direction of a single protein with two distinct functions to two subcellular sites does not require alternative splicing of the mRNA. Biochem J 345: 423-427, 2000.

52. Braiman L, Alt A, Kuroki T, Ohba M, Bak A, Tennenbaum T, Sampson SR. Activation of protein kinase C zeta induces serine phosphorylation of VAMP2 in the GLUT4 compartment and increases glucose transport in skeletal muscle. Mol Cell Biol 21: 7852-7861, 2001.

53. Brembeck FH, Rosario M, Birchmeier W. Balancing cell adhesion and Wnt signaling, the key role of beta-catenin. Curr Opin Genet Dev 16: 51-59, 2006.

54. Bressler R, Goldman S. A role of fatty acid oxidation in cardiac hypertrophy. Cardioscience 4: 133-142, 1993.

55. Broer S, Schneider HP, Broer A, Rahman B, Hamprecht B, Deitmer JW. Characterization of the monocarboxylate transporter 1 expressed in Xenopus laevis oocytes by changes in cytosolic $\mathrm{pH}$. Biochem J 333: 167-174, 1998.

56. Bronnikov GE, Aboulaich N, Vener AV, Stralfors P. Acute effects of insulin on the activity of mitochondrial GPAT1 in primary adipocytes. Biochem Biophys Res Commun 367: 201-207, 2008.

57. Brozinick JT Jr, Etgen GJ Jr, Yaspelkis BB 3rd, Ivy JL. Contraction-activated glucose uptake is normal in insulin-resistant muscle of the obese Zucker rat. J Appl Physiol 73: 382-387, 1992.

58. Brozinick JT Jr, Etgen GJ Jr, Yaspelkis BB 3rd, Ivy JL. The effects of muscle contraction and insulin on glucose transporter translocation in rat skeletal muscle. Biochem J 297: 539-545, 1994.

59. Brunaldi K, Simard JR, Kamp F, Rewal C, Asawakarn T, O'Shea P, Hamilton JA. Fluorescence assays for measuring fatty acid binding and transport through membranes. Methods Mol Biol 400: 237-255, 2007.

60. Bucci C, Parton RG, Mather IH, Stunnenberg H, Simons K, Hoflack B, Zerial M. The small GTPase rab5 functions as a regulatory factor in the early endocytic pathway. Cell 70: 715-728, 1992.

61. Burelle Y, Wambolt RB, Grist M, Parsons HL, Chow JC, Antler C, Bonen A, Keller A, Dunaway GA, Popov KM, Hochachka PW, Allard MF. Regular exercise is associated with a protective metabolic phenotype in the rat heart. Am J Physiol Heart Circ Physiol 287: H1055-H1063, 2004.

62. Burgomaster KA, Cermak NM, Phillips SM, Benton CR, Bonen A, Gibala MJ. Divergent response of metabolite transport proteins in human skeletal muscle after sprint interval training and detraining. Am J Physiol Regul Integr Comp Physiol 292: R1970R1976, 2007.

63. Campbell FM, Taffesse S, Gordon MJ, Dutta-Roy AK. Plasma membrane fatty-acid-binding protein in human placenta: identification and characterization. Biochem Biophys Res Commun 209: 1011-1017, 1995. 
64. Campbell SE, Tandon NN, Woldegiorgis G, Luiken JJFP, Glatz JFC, Bonen A. A novel function for FAT/CD36: involvement in long chain fatty acid transfer into the mitochondria. J Biol Chem 279: 36335-36341, 2004.

65. Carley AN, Atkinson LA, Bonen A, Harper ME, Kunnathu S, Lopaschuk GD, Severson DL. Mechanisms responsible for enhanced fatty acid utilization by perfused hearts from type $2 \mathrm{db} / \mathrm{db}$ mice. Arch Physiol Biochem 113: 65-75, 2007.

66. Carley AN, Severson DL. Fatty acid metabolism is enhanced in type 2 diabetic hearts. Biochim Biophys Acta 1734: 112-126, 2005.

67. Carling D, Clarke PR, Zammit VA, Hardie DG. Purification and characterization of the AMP-activated protein kinase. Copurification of acetyl-CoA carboxylase kinase and 3-hydroxy-3-methylglutaryl-CoA reductase kinase activities. Eur J Biochem 186: 129-136, 1989.

68. Cartee GD, Wojtaszewski JF. Role of Akt substrate of $160 \mathrm{kDa}$ in insulin-stimulated and contraction-stimulated glucose transport. Appl Physiol Nutr Metab 32: 557-566, 2007.

69. Cechetto JD, Sadacharan SK, Berk PD, Gupta RS. Immunogold localization of mitochondrial aspartate aminotransferase in mitochondria and on the cell surface in normal rat tissues. Histol Histopathol 17: 353-364, 2002.

70. Cha BS, Ciaraldi TP, Carter L, Nikoulina SE, Mudaliar S, Mukherjee R, Paterniti JR Jr, Henry RR. Peroxisome proliferator-activated receptor (PPAR) gamma and retinoid X receptor (RXR) agonists have complementary effects on glucose and lipid metabolism in human skeletal muscle. Diabetologia 44: 444-452, 2001.

71. Chabowski A, Chatham JC, Tandon NN, Calles-Escandon J, Glatz JFC, Luiken JJFP, Bonen A. Fatty acid transport and FAT/CD36 are increased in red but not in white muscle skeletal muscle of Zucker diabetic fatty (ZDF) rats. Am J Physiol Endocrinol Metab 291: E675-E682, 2006.

72. Chabowski A, Coort SL, Calles-Escandon J, Tandon NN, Glatz JF, Luiken JJ, Bonen A. Insulin stimulates fatty acid transport by regulating expression of FAT/CD36 but not FABPpm. Am J Physiol Endocrinol Metab 287: E781-E789, 2004.

73. Chabowski A, Coort SLM, Calles-Escandon J, Tandon NN, Glatz JFC, Luiken JJFP, Bonen A. The subcellular compartmentation of fatty acid transporters is regulated differently by insulin and by AICAR. FEBS Lett 579: 2428-2432, 2005.

74. Chabowski A, Górski J, Calles-Escandon J, Tandon NN, Bonen A. Hypoxia-induced fatty acid transporter translocation increases fatty acid transport and contributes to lipid accumulation in the heart. FEBS Lett 580: 3617-3623, 2006.

75. Chabowski A, Górski J, Luiken JJ, Glatz JF, Bonen A. Evidence for concerted action of FAT/CD36 and FABPpm to increase fatty acid transport across the plasma membrane. Prostaglandins Leukot Essent Fatty Acids 77: 345-353, 2007.

76. Chabowski A, Momken I, Coort SLM, Calles-Escandon J, Tandon NN, Glatz JFC, Luiken JJFP, Bonen A. Prolonged AMPK activation increases the expression of fatty acid transporters in cardiac myocytes and perfused hearts. Mol Cell Biochem 288: 201212, 2006.

77. Chavez JA, Holland WL, Bar J, Sandhoff K, Summers SA. Acid ceramidase overexpression prevents the inhibitory effects of saturated fatty acids on insulin signaling. J Biol Chem 280: 2014820153, 2005.

78. Chavez JA, Knotts TA, Wang LP, Li G, Dobrowsky RT, Florant GL, Summers SA. A role for ceramide, but not diacylglycerol, in the antagonism of insulin signal transduction by saturated fatty acids. J Biol Chem 278: 10297-10303, 2003.

79. Chen HC, Bandyopadhyay G, Sajan MP, Kanoh Y, Standaert M, Farese RV Jr, Farese RV. Activation of the ERK pathway and atypical protein kinase $\mathrm{C}$ isoforms in exercise- and aminoimidazole-4-carboxamide-1-beta-D-riboside (AICAR)-stimulated glucose transport. J Biol Chem 277: 23554-23562, 2002.

80. Chiu HC, Kovacs A, Blanton RM, Han X, Courtois M, Weinheimer CJ, Yamada KA, Brunet S, Xu H, Nerbonne JM, Welch MJ, Fettig NM, Sharp TL, Sambandam N, Olson KM, Ory DS, Schaffer JE. Transgenic expression of fatty acid transport protein 1 in the heart causes lipotoxic cardiomyopathy. Circ Res 96: 225233, 2005.
81. Cho H, Mu J, Kim JK, Thorvaldsen JL, Chu Q, Crenshaw EB, 3rd Kaestner KH, Bartolomei MS, Shulman GI, Birnbaum MJ. Insulin resistance and diabetes mellitus-like syndrome in mice lacking protein kinase Akt2 (PKB $\beta$ ). Science 292: 1728-1731, 2001.

82. Choi CS, Befroy DE, Codella R, Kim S, Reznick RM, Hwang YJ, Liu ZX, Lee HY, Distefano A, Samuel VT, Zhang D, Cline GW, Handschin C, Lin J, Petersen KF, Spiegelman BM, Shulman GI. Paradoxical effects of increased expression of PGClalpha on muscle mitochondrial function and insulin-stimulated muscle glucose metabolism. Proc Natl Acad Sci USA 105: 1992619931, 2008

83. Ciechanover A. The ubiquitin-proteasome proteolytic pathway. Cell 79: 13-21, 1994.

84. Civelek VN, Hamilton JA, Tornheim K, Kelly KL, Corkey BE. Intracellular $\mathrm{pH}$ in adipocytes: effects of free fatty acid diffusion across the plasma membrane, lipolytic agonists, and insulin. Proc Natl Acad Sci USA 93: 10139-10144, 1996.

85. Clarke DC, Miskovic D, Han XX, Calles-Escandon J, Glatz JFC, Luiken JJFP, Heikkila JJ, Bonen A. Overexpression of membrane associated fatty acid binding protein (FABPpm) in vivo increases fatty acid sarcolemmal transport and metabolism. Physiol Genomics 17: 31-37, 2004.

86. Coburn CT, Knapp FF Jr, Febbraio M, Beets AL, Silverstein RL, Abumrad NA. Defective uptake and utilization of long chain fatty acids in muscle and adipose tissue of CD36 knockout mice. J Biol Chem 275: 32523-32529, 2000.

87. Coe NR, Smith AJ, Frohnert BI, Watkins PA, Bernlohr DA. The fatty acid transport protein (FATP1) is a very long chain acyl-CoA synthetase. J Biol Chem 274: 36300-36304, 1999.

88. Cohen AW, Hnasko R, Schubert W, Lisanti MP. Role of caveolae and caveolins in health and disease. Physiol Rev 84: 1341-1379, 2004.

89. Collison M, Glazier AM, Graham D, Morton JJ, Dominiczak MH, Aitman TJ, Connell JM, Gould GW, Dominiczak AF. Cd36 and molecular mechanisms of insulin resistance in the strokeprone spontaneously hypertensive rat. Diabetes 49: 2222-2226, 2000.

90. Cooney GJ, Thompson AL, Furler SM, Ye J, Kraegen EW. Muscle long-chain acyl CoA esters and insulin resistance. Ann NY Acad Sci 967: 196-207, 2002.

91. Coort SLM, Hasselbaink DM, Koonen DPY, Willems J, Coumans WA, Chabowski A, van der Vusse GJ, Bonen A, Glatz JFC, Luiken JJFP. Enhanced sarcolemmal FAT/CD36 content and triacylglycerol storage in cardiac myocytes from obese Zucker rats. Diabetes 53: 1655-1663, 2004.

92. Coort SLM, Luiken JJFP, van Der Vusse GJ, Bonen A, Glatz JFC. Increased FAT (fatty acid translocase)/CD36-mediated longchain fatty acid uptake in cardiac myocytes from obese Zucker rats. Biochem Soc Trans 32: 83-85, 2004.

93. Coort SLM, Willems J, Coumans WA, van der Vusse GJ, Bonen A, Glatz JFC, Luiken JJFP. Sulfo- $N$-succinimidyl esters of long chain fatty acids specifically inhibit fatty acid translocase (FAT/CD36)-mediated cellular fatty acid uptake. Mol Cell Biochem 239: 213-219, 2002.

94. Corpeleijn E, Pelsers MM, Soenen S, Mensink M, Bouwman FG, Kooi ME, Saris WH, Glatz JF, Blaak EE. Insulin acutely upregulates protein expression of the fatty acid transporter CD36 in human skeletal muscle in vivo. J Physiol Pharmacol 59: 77-83, 2008.

95. Covey SD, Brunet RH, Gandhi SG, McFarlane N, Boreham DR, Gerber GE, Trigatti BL. Cholesterol depletion inhibits fatty acid uptake without affecting CD36 or caveolin-1 distribution in adipocytes. Biochem Biophys Res Commun 355: 67-71, 2007.

96. Criddle DN, Murphy J, Fistetto G, Barrow S, Tepikin AV, Neoptolemos JP, Sutton R, Petersen OH. Fatty acid ethyl esters cause pancreatic calcium toxicity via inositol trisphosphate receptors and loss of ATP synthesis. Gastroenterology 130: 781-793, 2006.

97. Cupp D, Kampf JP, Kleinfeld AM. Fatty acid-albumin complexes and the determination of the transport of long chain free fatty acids across membranes. Biochemistry 43: 4473-4481, 2004. 
98. Curtis BR, Aster RH. Incidence of the Nak(a)-negative platelet phenotype in African Americans is similar to that of Asians. Transfusion 36: 331-334, 1996.

99. Cushman SW, Goodyear LJ, Pilch PF, Ralston E, Galbo H, Ploug T, Kristiansen S, Klip A. Molecular mechanisms involved in GLUT4 translocation in muscle during insulin and contraction stimulation. Adv Exp Med Biol 441: 63-71, 1998.

100. De Bock K, Derave W, Eijnde BO, Hesselink MK, Koninckx E, Rose AJ, Schrauwen P, Bonen A, Richter EA, Hespel P. Effect of training in the fasted state on metabolic responses during exercise with carbohydrate intake. J Appl Physiol 104: 1045-1055, 2008.

101. De Feyter HM, van den Broek NM, Praet SF, Nicolay K, van Loon LJ, Prompers JJ. Early or advanced stage type 2 diabetes is not accompanied by in vivo skeletal muscle mitochondrial dysfunction. Eur J Endocrinol 158: 643-653, 2008.

102. Deneka M, Neeft M, van der Sluijs P. Regulation of membrane transport by rab GTPases. Crit Rev Biochem Mol Biol 38: 121-142, 2003.

103. DiRusso CC, Darwis D, Obermeyer T, Black PN. Functional domains of the fatty acid transport proteins: studies using protein chimeras. Biochim Biophys Acta 1781: 135-143, 2008

104. DiRusso CC, Li H, Darwis D, Watkins PA, Berger J, Black PN. Comparative biochemical studies of the murine fatty acid transport proteins (FATP) expressed in yeast. J Biol Chem 280: 16829-16837, 2005.

105. Doege H, Baillie RA, Ortegon AM, Tsang B, Wu Q, Punreddy S, Hirsch D, Watson N, Gimeno RE, Stahl A. Targeted deletion of FATP5 reveals multiple functions in liver metabolism: alterations in hepatic lipid homeostasis. Gastroenterology 130: 1245-1258, 2006.

106. Doege H, Grimm D, Falcon A, Tsang B, Storm TA, Xu H, Ortegon AM, Kazantzis M, Kay MA, Stahl A. Silencing of hepatic fatty acid transporter protein 5 in vivo reverses diet-induced non-alcoholic fatty liver disease and improves hyperglycemia. J Biol Chem 283: 22186-22192, 2008.

107. Doege H, Stahl A. Protein-mediated fatty acid uptake: novel insights from in vivo models. Physiology 21: 259-268, 2006.

108. Domin J, Pages F, Volinia S, Rittenhouse SE, Zvelebil MJ, Stein RC, Waterfield MD. Cloning of a human phosphoinositide 3-kinase with a $\mathrm{C} 2$ domain that displays reduced sensitivity to the inhibitor wortmannin. Biochem J 326: 139-147, 1997.

109. Dransfeld O, Uphues I, Sasson S, Schurmann A, Joost HG, Eckel J. Regulation of subcellular distribution of GLUT4 in cardiomyocytes: Rab4A reduces basal glucose transport and augments insulin responsiveness. Exp Clin Endocrinol Diabetes 108: 26-36, 2000.

110. Drover VA, Nguyen DV, Bastie CC, Darlington YF, Abumrad NA, Pessin JE, London E, Sahoo D, Phillips MC. CD36 mediates both cellular uptake of very long chain fatty acids and their intestinal absorption in mice. J Biol Chem 283: 13108-13115, 2008.

111. Dulubova I, Sugita S, Hill S, Hosaka M, Fernandez I, Sudhof TC, Rizo J. A conformational switch in syntaxin during exocytosis: role of munc18. EMBO J 18: 4372-4382, 1999.

112. Dyck DJ, Bonen A. Muscle contraction increases palmitate esterification and oxidation, triacylglycerol oxidation. Am J Physiol Endocrinol Metab 275: E888-E896, 1998.

113. Dyck DJ, Miskovic D, Code L, Luiken JJFP, Bonen A. Endurance training increases FFA oxidation and reduces triacylglycerol utilization in contracting rat soleus. Am J Physiol Endocrinol Metab 278: E778-E785, 2000.

114. Dyck DJ, Peters SJ, Glatz J, Górski J, Keizer H, Kiens B, Liu S, Richter EA, Spriet LL, van der Vusse GJ, Bonen A. Functional differences in lipid metabolism in resting skeletal muscle of various fiber types. Am J Physiol Endocrinol Metab 272: E340E351, 1997.

115. Dyck DJ, Steinberg G, Bonen A. Insulin increases FFA uptake and esterification but reduces lipid utilization in isolated contracting muscles. Am J Physiol Endocrinol Metab 281: E600-E607, 2001

116. Dzamko N, Schertzer JD, Ryall JG, Steel R, Macaulay SL, Wee S, Chen ZP, Michell BJ, Oakhill JS, Watt MJ, Jorgensen SB, Lynch GS, Kemp BE, Steinberg GR. AMPK-independent pathways regulate skeletal muscle fatty acid oxidation. J Physiol 586: 5819-5831, 2008.
117. Ehehalt R, Sparla R, Kulaksiz H, Herrmann T, Fullekrug J, Stremmel W. Uptake of long chain fatty acids is regulated by dynamic interaction of FAT/CD36 with cholesterol/sphingolipid enriched microdomains (lipid rafts). BMC Cell Biol 9: 45, 2008.

118. Eyre NS, Cleland LG, Mayrhofer G. FAT/CD36 expression alone is insufficient to enhance cellular uptake of oleate. Biochem Biophys Res Commun 370: 404-409, 2008.

119. Eyre NS, Cleland LG, Tandon NN, Mayrhofer G. Importance of the carboxyl terminus of FAT/CD36 for plasma membrane localization and function in long-chain fatty acid uptake. J Lipid Res 48: 528-542, 2007.

120. Faergeman NJ, DiRusso CC, Elberger A, Knudsen J, Black PN. Disruption of the Saccharomyces cerevisiae homologue to the murine fatty acid transport protein impairs uptake and growth on long-chain fatty acids. J Biol Chem 272: 8531-8538, 1997.

121. Fan X, Bradbury MW, Berk PD. Leptin and insulin modulate nutrient partitioning and weight loss in $o b / o b$ mice through regulation of long-chain fatty acid uptake by adipocytes. J Nutr 133: 2707-2715, 2003.

122. Farese RV. Function and dysfunction of aPKC isoforms for glucose transport in insulin-sensitive and insulin-resistant states. $A m J$ Physiol Endocrinol Metab 283: E1-E11, 2002.

123. Febbraio M, Abumrad NA, Hajjar DP, Sharma K, Cheng W, Frieda S, Pearce A, Silverstein RL. A null mutation in murine CD36 reveals an important role in fatty acid and lipoprotein metabolism. J Biol Chem 274: 19055-19062, 1999.

124. Febbraio M, Haj,jar DP, Silverstein RL. CD36: a class B scavenger receptor involved in angiogenesis, atherosclerosis, inflammation, and lipid metabolism. J Clin Invest 108: 785-791, 2001.

125. Febbraio M, Silverstein RL. CD36: implications in cardiovascular disease. Int J Biochem Cell Biol 39: 2012-2030, 2007.

126. Fitzgerald SM, Henegar JR, Brands MW, Henegar LK, Hall JE. Cardiovascular and renal responses to a high-fat diet in OsborneMendel rats. Am J Physiol Regul Integr Comp Physiol 281: R547R552, 2001.

127. Foster LJ, Klip A. Mechanism and regulation of GLUT-4 vesicle fusion in muscle and fat cells. Am J Physiol Cell Physiol 279: C877-C890, 2000.

128. Friedman JE, de Vente JE, Peterson RG, Dohm GL. Altered expression of muscle glucose transporter GLUT-4 in diabetic fatty Zucker rats (ZDF/Drt-fa). Am J Physiol Endocrinol Metab 261: E782-E788, 1991.

129. Frohnert BI, Hui TY, Bernlohr DA. Identification of a functional peroxisome proliferator-responsive element in the murine fatty acid transport protein gene. J Biol Chem 274: 3970-3977, 1999.

130. Fueger PJ, Hess HS, Posey KA, Bracy DP, Penceck RR, Charron MJ, Wasserman DH. Control of exercise-stimulated muscle glucose uptake by GLUT4 is dependent on glucose phosphorylation capacity in the conscious mouse. J Biol Chem 279: 50956-50961, 2004.

131. Fueger PT, Bracy DP, Malabanan CM, Pencek RR, Wasserman DH. Distributed control of glucose uptake by working muscles of conscious mice: roles of transport and phosphorylation. Am J Physiol Endocrinol Metab 286: E77-E84, 2004.

132. Fueger PT, Heikkinen S, Bracy DP, Malabanan CM, Pencek RR, Laakso M, Wasserman DH. Hexokinase II partial knockout impairs exercise-stimulated glucose uptake in oxidative muscles of mice. Am J Physiol Endocrinol Metab 285: E958-E963, 2003.

133. Fueger PT, Hess HS, Bracy DP, Pencek RR, Posey KA, Charron MJ, Wasserman DH. Regulation of insulin-stimulated muscle glucose uptake in the conscious mouse: role of glucose transport is dependent on glucose phosphorylation capacity. Endocrinology 145: 4912-4916, 2004.

134. Fueger PT, Li CY, Ayala JE, Shearer J, Bracy DP, Charron MJ, Rottman JN, Wasserman DH. Glucose kinetics and exercise tolerance in mice lacking the GLUT4 glucose transporter. J Physiol 582: 801-812, 2007.

135. Fueger PT, Shearer J, Bracy DP, Posey KA, Pencek RR, McGuinness OP, Wasserman DH. Control of muscle glucose uptake: test of the rate-limiting step paradigm in conscious, unrestrained mice. J Physiol 562: 925-935, 2005. 
136. Gargiulo CE, Stuhlsatz-Krouper SM, Schaffer JE. Localization of adipocyte long-chain fatty acyl CoA synthetase at the plasma membrane. J Lipid Res 40: 881-892, 1999.

137. Garofalo RS, Orena SJ, Rafidi K, Torchia AJ, Stock JL, Hildebrandt AL, Coskran T, Black SC, Brees DJ, Wicks JR, McNeish JD, Coleman KG. Severe diabetes, age-dependent loss of adipose tissue, and mild growth deficiency in mice lacking Akt2/ PKB beta. J Clin Invest 112: 197-208, 2003.

138. Gimeno RE, Hirsch DJ, Punreddy S, Sun Y, Ortegon AM, Wu H, Daniels T, Stricker-Krongrad A, Lodish HF, Stahl A. Targeted deletion of fatty acid transport protein-4 results in early embryonic lethality. J Biol Chem 278: 49512-49516, 2003.

139. Gimeno RE, Ortegon AM, Patel S, Punreddy S, Ge P, Sun Y, Lodish HF, Stahl A. Characterization of a heart-specific fatty acid transport protein. J Biol Chem 278: 16039-16044, 2003.

140. Glatz JFC, Bonen A, Ouwens DM, Luiken JJFP. Regulation of sarcolemmal transport of substrates in the healthy and diseased heart. Cardiovasc Drugs Ther 20: 471-476, 2006.

141. Glatz JFC, Storch J. Unravelling the significance of cellular fatty acid binding proteins. Curr Opin Lipidol 12: 267-274, 2001.

142. Glatz JFC, van der Vusse GJ. Cellular fatty acid-binding proteins: their function and physiological significance. Prog Lipid Res 35: 243-282, 1996

143. Glatz JFC, Van Nieuwenhoven FA, Luiken JJFP, Schaap FG, van der Vusse GJ. Role of membrane-associated and cytoplasmic fatty acid binding proteins and cellular fatty acid metabolism. Prostagl Leukotr Essent Fatty Acids 4-5: 373-378, 1997.

144. Glatz JFC, Veerkamp JH. Postnatal development of palmitate oxidation and mitochondrial enzyme activities in rat cardiac and skeletal muscle. Biochim Biophys Acta 711: 327-335, 1982

145. Górski J, Nawrocki A, Murthy M. Characterization of free and glyceride-esterified long chain fatty acids in different skeletal muscle types of the rat. Mol Cell Biochem 178: 113-118, 1998.

146. Gotoda T, Lituzaki Y, Kato N, Osuga J, Bihoreau MT, Murakami T, Yamori Y, Shimano H, Ishibashi S, Ymada N. Absence of Cd36 mutation in the original spontaneously hypertensive rats with insulin resistance. Nature Genet 22: 226-228, 1999.

147. Goudriaan JR, Dahlmans VE, Teusink B, Ouwens DM, Febbraio M, Maassen JA, Romijn JA, Havekes LM, Voshol PJ. CD36 deficiency increases insulin sensitivity in muscle, but induces insulin resistance in the liver in mice. J Lipid Res 44: 2270-2277, 2003.

148. Gratton JP, Bernatchez P, Sessa WC. Caveolae and caveolins in the cardiovascular system. Circ Res 94: 1408-1417, 2004.

149. Greaves J, Chamberlain LH. Palmitoylation-dependent protein sorting. J Cell Biol 176: 249-254, 2007.

150. Greenwalt DE, Scheck S, Rhinehart-Jones T. Heart CD36 expression is increased in murine models of diabetes and in mice fed a high fat diet. J Clin Invest 96: 1382-1388, 1995.

151. Guthmann F, Maehl P, Preiss J, Kolleck I, Rustow B. Ectoprotein kinase-mediated phosphorylation of FAT/CD36 regulates palmitate uptake by human platelets. Cell Mol Life Sci 59: 19992003, 2002

152. Habets DDJ. Regulation of Cardiac Long-Chain Fatty Acid and Glucose Metabolism: Studies With Cardiomyocytes From Genetically Manipulated Mice (PhD thesis). Maastricht: Maastricht University, 2008.

153. Habets DDJ, Coumans WA, Voshol PJ, den Boer MA, Febbraio M, Bonen A, Glatz JF, Luiken JJ. AMPK-mediated increase in myocardial long-chain fatty acid uptake critically depends on sarcolemmal CD36. Biochem Biophys Res Commun 355: 204-210, 2007.

154. Habets DDJ, Thurmond DC, Coumans WA, Bonen A, Glatz JFC, Luiken JJFP. Munc18 is not rate-limiting for glucose and long chain fatty acid uptake in the heart. Mol Cell Biochem. In press.

155. Hagenfeldt $\mathbf{L}$. Metabolism of free fatty acids and ketone bodies during exercise in normal and diabetic man. Diabetes 28, Suppl 1: $68-70,1979$

156. Hajri T, Abumrad NA. Fatty acid transport across membranes: relevance to nutrition and metabolic pathology. Annu Rev Nutr 22: 383-415, 2002.
157. Hajri T, Ibrahimi A, Coburn CT, Knapp FF Jr, Kurtz T, Pravenec M, Abumrad NA. Defective fatty acid uptake in the spontaneously hypertensive rat is a primary determinant of altered glucose metabolism, hyperinsulinemia, and myocardial hypertrophy. J Biol Chem 276: 23661-23666, 2001.

158. Hales CM, Vaerman JP, Goldenring JR. Rab11 family interacting protein 2 associates with Myosin $\mathrm{Vb}$ and regulates plasma membrane recycling. J Biol Chem 277: 50415-50421, 2002.

159. Hall AM, Smith AJ, Bernlohr DA. Characterization of the acylCoA synthetase activity of purified murine fatty acid transport protein 1. J Biol Chem 278: 43008-43013, 2003.

160. Hall AM, Wiczer BM, Herrmann T, Stremmel W, Bernlohr DA. Enzymatic properties of purified murine fatty acid transport protein 4 and analysis of acyl-CoA synthetase activities in tissues from FATP4 null mice. J Biol Chem 280: 11948-11954, 2005.

161. Hamilton J, Guo W, Kamp F. Mechanisms of cellular uptake of long-chain fatty acids: do we need cellular proteins? Mol Cell Biochem 239: 17-23, 2002.

162. Hamilton JA. New insights into the roles of proteins and lipids in membrane transport of fatty acids. Prostaglandins Leukot Essent Fatty Acids 77: 355-361, 2007.

163. Hamilton JA, Kamp F. How are free fatty acids transported in membranes? Is it by proteins or by free diffusion through the lipids? Diabetes 48: 2255-2269, 1999.

164. Han XX, Chabowski A, Tandon NN, Calles-Escandon J, Glatz JF, Luiken JJ, Bonen A. Metabolic challenges reveal impaired fatty acid metabolism and translocation of FAT/CD36 but not FABPpm in obese Zucker rat muscle. Am J Physiol Endocrinol Metab 293: E566E575, 2007.

165. Han XX, Fernando P, Bonen A. Denervation provokes greater reductions in insulin-stimulated glucose transport in muscle than severe diabetes. Mol Cell Biochem 210: 81-89, 2000.

166. Hardie DG. The AMP-activated protein kinase pathway-new players upstream and downstream. J Cell Sci 117: 5479-5487, 2004.

167. Hardie DG. AMPK: a key regulator of energy balance in the single cell and the whole organism. Int J Obes 32, Suppl 4: S7-S12, 2008.

168. Hardie DG. Minireview: the AMP-activated protein kinase cascade: the key sensor of cellular energy status. Endocrinology 144: 5179-5183, 2003.

169. Hardie DG, Hawley SA, Scott JW. AMP-activated protein kinase-development of the energy sensor concept. J Physiol 574: $7-15,2006$.

170. Hardie DG, Pan DA. Regulation of fatty acid synthesis and oxidation by the AMP-activated protein kinase. Biochem Soc Trans 30: 1064-1070, 2002.

171. Hardie DG, Salt IP, Hawley SA, Davies SP. AMP-activated protein kinase: an ultrasensitive system for monitoring cellular energy charge. Biochem J 338: 717-722, 1999.

172. Harmon CM, Abumrad NA. Binding of sulfosuccinimidyl fatty acids to adipocyte membrane proteins: isolation and amino-terminal sequence of an 88-kD protein implicated in transport of longchain fatty acids. J Membr Biol 133: 43-49, 1993.

173. Harmon CM, Luce P, Abumrad NA. Labelling of an $88 \mathrm{kDa}$ adipocyte membrane protein by sulpho- $N$-succinimidyl long-chain fatty acids: inhibition of fatty acid transport. Biochem Soc Trans 20: 811-813, 1992.

174. Harmon CM, Luce P, Beth AH, Abumrad NA. Labeling of adipocyte membranes by sulfo- $N$-succinimidyl derivatives of longchain fatty acids: inhibition of fatty acid transport. J Membr Biol 121: 261-268, 1991

175. Hata Y, Slaughter CA, Sudhof TC. Synaptic vesicle fusion complex contains unc-18 homologue bound to syntaxin. Nature 366: 347-351, 1993.

176. Hatch GM, Smith AJ, Xu FY, Hall AM, Bernlohr DA. FATP1 channels exogenous FA into 1,2,3-triacyl-sn-glycerol and downregulates sphingomyelin and cholesterol metabolism in growing 293 cells. J Lipid Res 43: 1380-1389, 2002.

177. Hatmi M, Gavaret JM, Elalamy I, Vargaftig BB, Jacquemin C. Evidence for cAMP-dependent platelet ectoprotein kinase activity that phosphorylates platelet glycoprotein IV (CD36). J Biol Chem 271: 24776-24780, 1996.

178. Hawley SA, Boudeau J, Reid JL, Mustard KJ, Udd L, Makela TP, Alessi DR, Hardie DG. Complexes between the LKB1 tumor 
suppressor, STRAD alpha/beta and MO25 alpha/beta are upstream kinases in the AMP-activated protein kinase cascade. J Biol 2: 28, 2003.

179. Hawley SA, Pan DA, Mustard KJ, Ross L, Bain J, Edelman AM, Frenguelli BG, Hardie DG. Calmodulin-dependent protein kinase kinase-beta is an alternative upstream kinase for AMPactivated protein kinase. Cell Metab 2: 9-19, 2005.

180. He J, Kelley DE. Muscle glycogen content in type 2 diabetes mellitus. Am J Physiol Endocrinol Metab 287: E1002-E1007, 2004.

181. Heather LC, Cole MA, Lygate CA, Evans RD, Stuckey DJ, Murray AJ, Neubauer S, Clarke K. Fatty acid transporter levels and palmitate oxidation rate correlate with ejection fraction in the infarcted rat heart. Cardiovasc Res 72: 430-437, 2006.

182. Hegarty BD, Cooney GJ, Kraegen EW, Furler SM. Increased efficiency of fatty acid uptake contributes to lipid accumulation in skeletal muscle of high fat-fed insulin-resistant rats. Diabetes 51: 1477-1484, 2002.

183. Heinzer AK, Watkins PA, Lu JF, Kemp S, Moser AB, Li YY, Mihalik S, Powers JM, Smith KD. A very long-chain acyl-CoA synthetase-deficient mouse and its relevance to X-linked adrenoleukodystrophy. Hum Mol Genet 12: 1145-1154, 2003.

184. Henriksen EJ, Bourney RE, Rodnick KJ, Koranyi L, Permutt MA, Holloszy JO. Glucose transporter protein content and glucose transport capacity in rat skeletal muscles. Am J Physiol Endocrinol Metab 259: E593-E598, 1990.

185. Herrmann T, Buchkremer F, Gosch I, Hall AM, Bernlohr DA, Stremmel W. Mouse fatty acid transport protein 4 (FATP4): characterization of the gene and functional assessment as a very long chain acyl-CoA synthetase. Gene 270: 31-40, 2001.

186. Herrmann T, Grone HJ, Langbein L, Kaiser I, Gosch I, Bennemann U, Metzger D, Chambon P, Stewart AF, Stremmel W. Disturbed epidermal structure in mice with temporally controlled fatp4 deficiency. J Invest Dermatol 125: 1228-1235, 2005.

187. Herrmann T, van der Hoeven F, Grone HJ, Stewart AF, Langbein L, Kaiser I, Liebisch G, Gosch I, Buchkremer F, Drobnik W, Schmitz G, Stremmel W. Mice with targeted disruption of the fatty acid transport protein 4 (Fatp 4, Slc27a4) gene show features of lethal restrictive dermopathy. J Cell Biol 161: 1105-1115, 2003.

188. Hertzel AV, Bernlohr DA. The mammalian fatty acid-binding protein multigene family: molecular and genetic insights into function. Trends Endocrinol Metab 11: 175-180, 2000.

189. Hevener A, Reichart D, Janez A, Olefsky J. Female rats do not exhibit free fatty acid-induced insulin resistance. Diabetes 51: 1907-1912, 2002.

190. Hirai T, Fukui Y, Motojima K. PPARalpha agonists positively and negatively regulate the expression of several nutrient/drug transporters in mouse small intestine. Biol Pharm Bull 30: 2185-2190, 2007.

191. Hirsch D, Stahl A, Lodish HF. A family of fatty acid transporters conserved from mycobacterium to man. Proc Natl Acad Sci USA 95: 8625-8629, 1998.

192. Hoebe K, Georgel P, Rutschmann S, Du X, Mudd S, Crozat K, Sovath S, Shamel L, Hartung T, Zahringer U, Beutler B. CD36 is a sensor of diacylglycerides. Nature 433: 523-527, 2005.

193. Holland WL, Brozinick JT, Wang LP, Hawkins ED, Sargent KM, Liu Y, Narra K, Hoehn KL, Knotts TA, Siesky A, Nelson DH, Karathanasis SK, Fontenot GK, Birnbaum MJ, Summers SA. Inhibition of ceramide synthesis ameliorates glucocorticoid-, saturated-fat-, obesity-induced insulin resistance. Cell Metab 5: 167-179, 2007.

194. Holland WL, Knotts TA, Chavez JA, Wang LP, Hoehn KL, Summers SA. Lipid mediators of insulin resistance. Nutr Rev 65: S39-S46, 2007.

195. Holloszy JO. Skeletal muscle "mitochondrial deficiency" does not mediate insulin resistance. Am J Clin Nutr 89: 463S-466S, 2009.

196. Holloszy JO, Booth FW. Biochemical adaptations to endurance exercise in muscle. Annu Rev Physiol 38: 273-291, 1976.

197. Holloway GP, Benton CR, Mullen KL, Yoshida Y, Snook LA, Han XX, Glatz JFC, Luiken JJFP, Lally J, Dyck DJ, Bonen A. In obese rat muscle transport of palmitate is increased and is channeled to triacylglycerol storage despite an increase in mitochondrial palmitate oxidation. Am J Physiol Endocrinol Metab 296: E738-E747, 2009.
198. Holloway GP, Bezaire V, Heigenhauser GJ, Tandon NN, Glatz JF, Luiken JJ, Bonen A, Spriet LL. Mitochondrial long chain fatty acid oxidation, fatty acid translocase/CD36 content and carnitine palmitoyltransferase 1 activity in human skeletal muscle during aerobic exercise. J Physiol 571: 201-210, 2006.

199. Holloway GP, Jain SS, Bezaire V, Han XX, Glatz JFC, Luiken JJFP, Harper ME, Bonen A. FAT/CD36 null mice reveal that mitochondrial FAT/CD36 is required to up-regulate mitochondrial fatty acid oxidation in contracting muscle. Am J Physiol Regul Integr Comp Physiol 297: R960-R967, 2009.

200. Holloway GP, Lally J, Nickerson JG, Alkhateeb H, Snook LA, Heigenhauser GJ, Calles-Escandon J, Glatz JF, Luiken JJ, Spriet LL, Bonen A. Fatty acid binding protein facilitates sarcolemmal fatty acid transport but not mitochondrial oxidation in rat and human skeletal muscle. J Physiol 582: 393-405, 2007.

201. Holloway GP, Luiken JJFP, Glatz JFC, Spriet LL, Bonen A. Contribution of FAT/CD36 to the regulation of skeletal muscle fatty acid oxidation: an overview. Acta Physiol 194: 293-309, 2008.

202. Holloway GP, Perry CG, Thrush AB, Heigenhauser GJ, Dyck DJ, Bonen A, Spriet LL. PGC1 $\alpha$ relationship with skeletal muscle palmitate oxidation is not present with obesity, despite maintained PGC1 $\alpha$ and PGC1 $\beta$ protein. Am J Physiol Endocrinol Metab 294: E1060-E1069, 2008.

203. Holloway GP, Thrush AB, Heigenhauser GJ, Tandon NN, Dyck DJ, Bonen A, Spriet LL. Skeletal muscle mitochondrial FAT/CD36 and palmitate oxidation are not decreased in obese women. Am J Physiol Endocrinol Metab 292: E1782-E1789, 2007.

204. Hong SP, Leiper FC, Woods A, Carling D, Carlson M. Activation of yeast Snf1 and mammalian AMP-activated protein kinase by upstream kinases. Proc Natl Acad Sci USA 100: 8839-8843, 2003.

205. Hoosdally SJ, Andress EJ, Wooding C, Martin CA, Linton KJ. The human scavenger receptor CD36: glycosylation status and its role in trafficking and function. J Biol Chem 284: 16277-16288, 2009.

206. Huang J, Imamura T, Olefsky JM. Insulin can regulate GLUT4 internalization by signaling to Rab5 and the motor protein dynein. Proc Natl Acad Sci USA 98: 13084-13089, 2001.

207. Huang S, Czech MP. The GLUT4 glucose transporter. Cell Metab 5: 237-252, 2007.

208. Huang YS, Richter JD. Regulation of local mRNA translation. Curr Opin Cell Biol 16: 308-313, 2004.

209. Hubbard B, Doege H, Punreddy S, Wu H, Huang X, Kaushik VK, Mozell RL, Byrnes JJ, Stricker-Krongrad A, Chou CJ, Tartaglia LA, Lodish HF, Stahl A, Gimeno RE. Mice deleted for fatty acid transport protein 5 have defective bile acid conjugation and are protected from obesity. Gastroenterology 130: 1259-1269, 2006.

210. Hulver MW, Berggren JR, Cortright RN, Dudek RW, Thompson RP, Pories WJ, MacDonald KG, Cline GW, Shulman GI, Dohm GL, Houmard JA. Skeletal muscle lipid metabolism with obesity. Am J Physiol Endocrinol Metab 284: E741-E747, 2003.

211. Huynh M, Luiken JJ, Coumans W, Bell RC. Dietary fructose during the suckling period increases body weight and fatty acid uptake into skeletal muscle in adult rats. Obesity 16: 1755-1762, 2008.

212. Ibrahimi A, Abumrad NA. Role of CD36 in membrane transport of long-chain fatty acids. Curr Opin Clin Nutr Metab Care 5: 139-145, 2002.

213. Ibrahimi A, Bonen A, Blinn WD, Hajri T, Li X, Zhong K, Cameron R, Abumrad NA. Muscle-specific overexpression of FAT/CD36 enhances fatty acid oxidation by contracting muscles, reduces plasma triglycerides and fatty acids, and increases plasma glucose and insulin. J Biol Chem 274: 26761-26766, 1999.

214. Ibrahimi A, Sfeir Z, Magharaine H, Amri EZ, Grimaldi P, Abumrad NA. Expression of the CD36 homolog (FAT) in fibroblast cells: effects on fatty acid transport. Proc Natl Acad Sci USA 93: 2646-2651, 1996.

215. Ikeda S, Miyazaki H, Nakatani T, Kai Y, Kamei Y, Miura S, Tsuboyama-Kasaoka N, Ezaki O. Up-regulation of SREBP-1c and lipogenic genes in skeletal muscles after exercise training. Biochem Biophys Res Commun 296: 395-400, 2002.

216. Irie H, Krukenkamp IB, Brinkmann JF, Gaudette GR, Saltman AE, Jou W, Glatz JF, Abumrad NA, Ibrahimi A. Myocar- 
dial recovery from ischemia is impaired in CD36-null mice and restored by myocyte CD36 expression or medium-chain fatty acids. Proc Natl Acad Sci USA 100: 6819-6824, 2003.

217. Isola LM, Zhou SL, Kiang CL, Stump DD, Bradbury MW, Berk PD. 3T3 fibroblasts transfected with a cDNA for mitochondrial aspartate aminotransferase express plasma membrane fatty acidbinding protein and saturable fatty acid uptake. Proc Natl Acad Sci USA 92: 9866-9870, 1995.

218. Jain S, Glatz JFC, Luiken JJFP, Bonen A. Regulation of insulinand contraction-stimuated fatty acid transporters: FAT/CD36, FABPpm, FATP1, FATP4 and FATP6. International Conference on the Bioscience of Lipids, Maastricht, The Netherlands, 2008.

219. Jain SS, Chabowski A, Snook LA, Schwenk RW, Glatz JF, Luiken JJ, Bonen A. Additive effects of insulin and muscle contraction on fatty acid transport and fatty acid transporters, FAT/ CD36, FABPpm, FATP1, 4 and 6. FEBS Lett 583: 2294-2300, 2009.

220. Jenkins AB, Storlien LH, Chisholm DJ, Kraegen EW. Effects of nonesterified fatty acid availability on tissue-specific glucose utilization in rats in vivo. J Clin Invest 82: 293-299, 1988.

221. Jenkins AB, Storlien LH, Cooney GJ, Denyer GS, Caterson ID, Kraegen EW. Effects of blockade of fatty acid oxidation on whole body and tissue-specific glucose metabolism in rats. $A m J$ Physiol Endocrinol Metab 265: E592-E600, 1993.

222. Jensen TE, Rose AJ, Hellsten Y, Wojtaszewski JF, Richter EA. Caffeine-induced $\mathrm{Ca}^{2+}$ release increases AMPK-dependent glucose uptake in rodent soleus muscle. Am J Physiol Endocrinol Metab 293: E286-E292, 2007.

223. Jensen TE, Rose AJ, Jorgensen SB, Brandt N, Schjerling P, Wojtaszewski JF, Richter EA. Possible CaMKK-dependent regulation of AMPK phosphorylation and glucose uptake at the onset of mild tetanic skeletal muscle contraction. Am J Physiol Endocrinol Metab 292: E1308-E1317, 2007.

224. Jochen A, Hays J. Purification of the major substrate for palmitoylation in rat adipocytes: N-terminal homology with CD36 and evidence for cell surface acylation. J Lipid Res 34: 1783-1792, 1993.

225. Jochen AL, Hays J, Mick G. Inhibitory effects of cerulenin on protein palmitoylation and insulin internalization in rat adipocytes. Biochim Biophys Acta 1259: 65-72, 1995.

226. Johnson AC, Stahl A, Zager RA. Triglyceride accumulation in injured renal tubular cells: alterations in both synthetic and catabolic pathways. Kidney Int 67: 2196-2209, 2005.

227. Jørgensen SB, Viollet B, Andreelli F, Frosig C, Birk JB, Schjerling P, Vaulont S, Richter EA, Wojtaszewski JF. Knockout of the alpha 2 but not alpha1 5'-AMP-activated protein kinase isoform abolishes 5-aminoimidazole-4-carboxamide-1-beta-4-ribofuranoside but not contraction-induced glucose uptake in skeletal muscle. J Biol Chem 279: 1070-1079, 2004.

228. Kamp F, Guo W, Souto R, Pilch PF, Corkey BE, Hamilton JA. Rapid flip-flop of oleic acid across the plasma membrane of adipocytes. J Biol Chem 278: 7988-7995, 2003.

229. Kamp F, Hamilton JA. pH gradients across phospholipid membranes caused by fast flip-flop of un-ionized fatty acids. Proc Natl Acad Sci USA 89: 11367-11370, 1992.

230. Kamp F, Zakim D, Zhang F, Noy N, Hamilton JA. Fatty acid flip-flop in phospholipid bilayers is extremely fast. Biochemistry 34: 11928-11937, 1995.

231. Kampf JP, Cupp D, Kleinfeld AM. Different mechanisms of free fatty acid flip-flop and dissociation revealed by temperature and molecular species dependence of transport across lipid vesicles. J Biol Chem 281: 21566-21574, 2006.

232. Kampf JP, Kleinfeld AM. Fatty acid transport in adipocytes monitored by imaging intracellular free fatty acid levels. $J$ Biol Chem 279: 35775-35780, 2004.

233. Kampf JP, Kleinfeld AM. Is membrane transport of FFA mediated by lipid, protein, or both? An unknown protein mediates free fatty acid transport across the adipocyte plasma membrane. Physiology 22: 7-14, 2007.

234. Kampf JP, Parmley D, Kleinfeld AM. Free fatty acid transport across adipocytes is mediated by an unknown membrane protein pump. Am J Physiol Endocrinol Metab 293: E1207-E1214, 2007.

235. Katz AM, Messineo FC. Lipid-membrane interactions and the pathogenesis of ischemic damage in the myocardium. Circ Res 48: $1-16,1981$.
236. Keizer HA, Schaart G, Tandon NN, Glatz JF, Luiken JJ. Subcellular immunolocalisation of fatty acid translocase (FAT)/CD36 in human type-1 and type-2 skeletal muscle fibres. Histochem Cell Biol 121: 101-107, 2004.

237. Kelley DE, He J, Menshikova EV, Ritov VB. Dysfunction of mitochondria in human skeletal muscle in type 2 diabetes. Diabetes 51: 2944-2950, 2002.

238. Kelley DE, Mandarino LJ. Fuel selection in human skeletal muscle in insulin resistance. A reexamination. Diabetes 49: 677-683, 2000.

239. Kelly KR, Sung CK, Abbott MJ, Turcotte LP. Phosphatidylinositol 3-kinase-dependent insulin regulation of long-chain fatty acid (LCFA) metabolism in L6 muscle cells: involvement of atypical protein kinase C-zeta in LCFA uptake but not oxidation. $J$ Endocrinol 198: 375-384, 2008.

240. Kerner J, Hoppel C. Fatty acid import into mitochondria. Biochim Biophys Acta 1486: 1-17, 2000.

241. Khan NA, Besnard P. Oro-sensory perception of dietary lipids: new insights into the fat taste transduction. Biochim Biophys Acta 1791: 149-155, 2009.

242. Kiens B. Skeletal muscle lipid metabolism in exercise and insulin resistance. Physiol Rev 86: 205-243, 2006.

243. Kiens B, Kristiansen S, Jensen P, Richter EA, Turcotte LP. Membrane associated fatty acid binding protein (FABPpm) in human skeletal muscle is increased by endurance training. Biochem Biophys Res Commun 231: 463-465, 1997.

244. Kiens B, Roepstorff C, Glatz JF, Bonen A, Schjerling P, Knudsen J, Nielsen JN. Lipid-binding proteins and lipoprotein lipase activity in human skeletal muscle: influence of physical activity and gender. J Appl Physiol 97: 1209-1218, 2004.

245. Kim JK, Gimeno RE, Higashimori T, Kim HJ, Choi H, Punreddy S, Mozell RL, Tan G, Stricker-Krongrad A, Hirsch DJ, Fillmore JJ, Liu ZX, Dong J, Cline G, Stahl A, Lodish HF, Shulman GI. Inactivation of fatty acid transport protein 1 prevents fat-induced insulin resistance in skeletal muscle. J Clin Invest 113: 756-763, 2004.

246. Kim JY, Hickner RL, Cortright RN, Dohm GL, Houmard JA. Lipid oxidation is reduced in obese human skeletal muscle. Am J Physiol Endocrinol Metab 279: E1039-E1044, 2000.

247. Kim SO, Hasham MI, Katz S, Pelech SL. Insulin-regulated protein kinases during postnatal development of rat heart. $J$ Cell Biochem 71: 328-339, 1998.

248. King KL, Stanley WC, Rosca M, Kerner J, Hoppel CL, Febbraio M. Fatty acid oxidation in cardiac and skeletal muscle mitochondria is unaffected by deletion of CD36. Arch Biochem Biophys 467: 234-238, 2007.

249. Klein K, Steinberg R, Fiethen B, Overath P. Fatty acid degradation in Escherichia coli. An inducible system for the uptake of fatty acids and further characterization of old mutants. Eur J Biochem 19: 442-450, 1971.

250. Kleinfeld AM, Chu P, Romero C. Transport of long-chain native fatty acids across lipid bilayer membranes indicates that transbilayer flip-flop is rate limiting. Biochemistry 36: 14146-14158, 1997.

251. Kleinfeld AM, Chu P, Storch J. Flip-flop is slow and rate limiting for the movement of long chain anthroyloxy fatty acids across lipid vesicles. Biochemistry 36: 5702-5711, 1997.

252. Koonen DPY, Benton CR, Arumugam Y, Tandon NN, CallesEscandon J, Glatz JFC, Luiken JJFP, Bonen A. Different mechanisms can alter fatty acid transport when muscle contractile activity is chronically altered. Am J Physiol Endocrinol Metab 286: E1042-E1049, 2004.

253. Koonen DPY, Coumans WA, Arumugam Y, Bonen A, Glatz JFC, Luiken JJFP. Giant membrane vesicles as a model to study cellular substrate uptake dissected from metabolism. Mol Cell Biochem 239: 121-130, 2002.

254. Koonen DPY, Febbraio M, Bonnet S, Nagendran J, Young ME, Michelakis ED, Dyck JR. CD36 expression contributes to ageinduced cardiomyopathy in mice. Circulation 116: 2139-2147, 2007.

255. Koves TR, Ussher JR, Noland RC, Slentz D, Mosedale M, Ilkayeva O, Bain J, Stevens R, Dyck JR, Newgard CB, Lopaschuk GD, Muoio DM. Mitochondrial overload and incomplete 
fatty acid oxidation contribute to skeletal muscle insulin resistance. Cell Metab 7: 45-56, 2008.

256. Koyama K, Chen G, Lee Y, Unger RH. Tissue triglycerides, insulin resistance, and insulin production: implications for hyperinsulinemia of obesity. Am J Physiol Endocrinol 273: E708-E713, 1997.

257. Kramer HF, Witczak CA, Fujii N, Jessen N, Taylor EB, Arnolds DE, Sakamoto K, Hirshman MF, Goodyear LJ. Distinct signals regulate AS160 phosphorylation in response to insulin, AICAR, and contraction in mouse skeletal muscle. Diabetes 55: 2067-2076, 2006.

258. Kuang M, Febbraio M, Wagg C, Lopaschuk GD, Dyck JR. Fatty acid translocase/CD36 deficiency does not energetically or functionally compromise hearts before or after ischemia. Circulation 109: 1550-1557, 2004.

259. Kudo N, Gillespie JG, Kung L, Witters LA, Schulz R, Clanachan AS, Lopaschuk GD. Characterization of 5'AMP-activated protein kinase activity in the heart and its role in inhibiting acetylCoA carboxylase during reperfusion following ischemia. Biochim Biophys Acta 1301: 67-75, 1996.

260. Kusaka Y, Tanaka T, Okamoto F, Terasaki F, Matsunaga Y, Miyazaki H, Kawamura K. Effect of sulfo- $N$-succinimidyl palmitate on the rat heart: myocardial long-chain fatty acid uptake and cardiac hypertrophy. J Mol Cell Cardiol 27: 1605-1612, 1995.

261. Lauritzen HP, Ploug T, Prats C, Tavare JM, Galbo H. Imaging of insulin signaling in skeletal muscle of living mice shows major role of T-tubules. Diabetes 55: 1300-1306, 2006.

262. Lee K, Godeau B, Fromont P, Plonquet A, Debili N, Bachir D, Reviron D, Gourin J, Fernandez E, Galacteros F, Bierling P. CD36 deficiency is frequent and can cause platelet immunization in Africans. Transfusion 39: 873-879, 1999.

263. Lee W, Ryu J, Spangler RA, Jung CY. Modulation of GLUT4 and GLUT1 recycling by insulin in rat adipocytes: kinetic analysis based on the involvement of multiple intracellular compartments. Biochemistry 39: 9358-9366, 2000.

264. Lemieux K, Han XX, Dombrowski L, Bonen A, Marette A. The transferrin receptor defines two distinct contraction-responsive GLUT4 vesicle populations. Diabetes 49: 183-189, 2000.

265. Lemieux K, Konrad D, Klip A, Marette A. The AMP-activated protein kinase activator AICAR does not induce GLUT4 translocation to transverse tubules but stimulates glucose uptake and p38 mitogen-activated protein kinases alpha and beta in skeletal muscle. FASEB J 17: 1658-1665, 2003.

266. Lewis SE, Listenberger LL, Ory D, Schaffer JR. Membrane topology of the murine fatty acid transport protein 1. J Biol Chem 276: 37042-37050, 2001.

267. Li J, Hu X, Selvakumar P, Russell RR, 3rd Cushman SW, Holman GD, Young LH. Role of the nitric oxide pathway in AMPK-mediated glucose uptake and GLUT4 translocation in heart muscle. Am J Physiol Endocrinol Metab 287: E834-E841, 2004.

268. Lobo S, Wiczer BM, Smith AJ, Hall AM, Bernlohr DA. Fatty acid metabolism in adipocytes: functional analysis of fatty acid transport proteins 1 and 4. J Lipid Res 48: 609-620, 2007.

269. Long YC, Barnes BR, Mahlapuu M, Steiler TL, Martinsson S, Leng Y, Wallberg-Henriksson H, Andersson L, Zierath JR. Role of AMP-activated protein kinase in the coordinated expression of genes controlling glucose and lipid metabolism in mouse white skeletal muscle. Diabetologia 48: 2354-2364, 2005.

270. Lopaschuk GD. Metabolic abnormalities in the diabetic heart. Heart Fail Rev 7: 149-159, 2002.

271. Love-Gregory L, Sherva R, Sun L, Wasson J, Schappe T, Doria A, Rao DC, Hunt SC, Klein S, Neuman RJ, Permutt MA, Abumrad NA. Variants in the CD36 gene associate with the metabolic syndrome and high-density lipoprotein cholesterol. Hum Mol Genet 17: 1695-1704, 2008.

272. Luiken JJFP, Arumugam Y, Bell RC, Calles-Escandon J, Tandon NN, Glatz JFC, Bonen A. Changes in fatty acid transport and transporters are related to the severity of insulin deficiency. Am J Physiol Endocrinol Metab 282: E612-E21, 2002.

273. Luiken JJFP, Arumugam Y, Dyck DJ, Bell RC, Pelsers ML, Turcotte LP, Tandon NN, Glatz JFC, Bonen A. Increased rates of fatty acid uptake and plasmalemmal fatty acid transporters in obese Zucker rats. J Biol Chem 276: 40567-40573, 2001.
274. Luiken JJFP, Coort SL, Willems J, Coumans WA, Bonen A, Glatz JFC. Dipyridamole alters cardiac substrate preference by inducing translocation of FAT/CD36, but not that of GLUT4. Mol Pharmacol 65: 639-645, 2004.

275. Luiken JJFP, Coort SLM, Koonen DPY, Bonen A, Glatz JFC. Signalling components involved in contraction-inducible substrate uptake into cardiac myocytes. Proc Nutr Soc 63: 251-258, 2004.

276. Luiken JJFP, Coort SLM, Koonen DPY, van der Horst DJ, Bonen A, Zorzano A, Glatz JFC. Regulation of cardiac longchain fatty acid and glucose uptake by translocation of substrate transporters. Eur J Physiol 448: 1-15, 2004.

277. Luiken JJFP, Coort SML, Willems J, Coumans WA, Bonen A, van der Vusse GJ, Glatz JFC. Contraction-induced fatty acid translocase/CD36 translocation in rat cardiac myocytes is mediated through AMP-activated protein kinase signaling. Diabetes 52: 1627$1634,2003$.

278. Luiken JJFP, Dyck DJ, Han XX, Tandon NN, Arumugam Y, Glatz JFC, Bonen A. Insulin induces the translocation of the fatty acid transporter FAT/CD36 to the plasma membrane. Am J Physiol Endocrinol Metab 282: E491-E495, 2002.

279. Luiken JJFP, Han XX, Dyck DJ, Bonen A. Coordinately regulated expression of FAT/CD36 and FACS1 in rat skeletal muscle. Mol Cell Biochem 223: 61-69, 2001.

280. Luiken JJFP, Koonen DPY, Coumans WA, Pelsers MMAL, Binas B, Bonen A, Glatz JFC. Long chain fatty acid uptake by skeletal muscles is impaired in homozygous, but not heterozygous, H-FABP null mice. Lipids 38: 491-496, 2003.

281. Luiken JJFP, Koonen DPY, Willems J, Zorzano A, Fischer Y, van der Vusse GJ, Bonen A, Glatz JFC. Insulin stimulates long-chain fatty acid uilization by rat cardiac myocytes through cellular redistribution of FAT/CD36. Diabetes 51: 3113-3119, 2002.

282. Luiken JJFP, Momken I, Habets DD, El Hasnaoui M, Coumans WA, Koonen DP, Glatz JFC, Bonen A. Arsenite modulates cardiac substrate preference by translocation of GLUT4, but not of CD36, independent of MAPK signaling. Endocrinology 147: 52055216,2006

283. Luiken JJFP, Niessen HEC, Coort SM, Hoebers N, Coumans WA, Schwenk RW, Bonen A, Glatz JFC. Etomoxir-induced partial CPT-I inhibition in vivo does not alter cardiac long-chain fatty acid uptake and oxidation rates. Biochem J. 419: 447-455, 2009.

284. Luiken JJFP, Ouwens DM, Habets DD, van der Zon GC, Coumans WA, Schwenk RW, Bonen A, Glatz JFC. Permissive action of protein kinase C-zeta in insulin-induced CD36- and GLUT4 translocation in cardiac myocytes. J Endocrinol 201: 199-209, 2009.

285. Luiken JJFP, Schaap FG, van Nieuwenhoven FA, van der Vusse GJ, Bonen A, Glatz JFC. Cellular fatty acid transport in heart and skeletal muscle as facilitated by proteins. Lipids 34, Suppl: S169-S175, 1999.

286. Luiken JJFP, Turcotte LP, Bonen A. Protein-mediated palmitate uptake and expression of fatty acid transport proteins in heart giant vesicles. J Lipid Res 40: 1007-1016, 1999.

287. Luiken JJFP, van Nieuwenhoven FA, America G, van der Vusse GJ, Glatz JFC. Uptake and metabolism of palmitate by isolated cardiac myocytes from adult rats: Involvement of sarcolemmal proteins. J Lipid Res 38: 745-758, 1997.

288. Luiken JJFP, Vertommen D, Coort SL, Habets DD, El Hasnaoui M, Pelsers MM, Viollet B, Bonen A, Hue L, Rider MH, Glatz JFC. Identification of protein kinase D as a novel contraction-activated kinase linked to GLUT4-mediated glucose uptake, independent of AMPK. Cell Signal 20: 543-556, 2008.

289. Luiken JJFP, Willems J, Coort SL, Coumans WA, Bonen A, van der Vusse GJ, Glatz JFC. Effects of cAMP modulators on long-chain fatty-acid uptake and utilization by electrically stimulated rat cardiac myocytes. Biochem $J$ 367: 881-887, 2002.

290. Luiken JJFP, Willems J, van der Vusse GJ, Glatz JFC. Electrostimulation enhances FAT/CD36-mediated long-chain fatty acid uptake by isolated rat cardiac myocytes. Am J Physiol Endocrinol Metab 281: E704-E712, 2001.

291. Madrazo JA, Kelly DP. The PPAR trio: regulators of myocardial energy metabolism in health and disease. J Mol Cell Cardiol 44: 968-975, 2008 . 
292. Mangroo D, Gerber GE. Fatty acid uptake in Escherichia coli: regulation by recruitment of fatty acyl-CoA synthetase to the plasma membrane. Biochem Cell Biol 71: 51-56, 1993.

293. Martin G, Schoonjans K, Lefebvre AM, Staels B, Auwerx J. Coordinate regulation of the expression of the fatty acid transport protein and acyl-CoA synthetase genes by PPARalpha and PPARgamma activators. J Biol Chem 272: 28210-28217, 1997.

294. Matsuno K, Diaz-Ricard M, Montgomery RR, Aster T, Jamieson GA, Tandon NN. Inhibition of platelet adhesion to collagen by monoclonal anti CD36 antibodies. Br J Haematol 92: 960-967, 1996.

295. McCullagh KJA, Juel C, O'Brien M, Bonen A. Chronic muscle stimulation increases lactate transport in rat skeletal muscle. Mol Cell Biochem 156: 51-57, 1996.

296. McGarry JD, Brown NF. The mitochondrial carnitine palmitoyltransferase system. From concept to molecular analysis. Eur J Biochem 244: 1-14, 1997.

297. McMahon HT, Mills IG. COP and clathrin-coated vesicle budding: different pathways, common approaches. Curr Opin Cell Biol 16: 379-391, 2004.

298. Megeney LA, Neufer PD, Dohm GL, Tan MH, Blewett CA, Elder GCB, Bonen A. Effects of muscle activity and fiber composition on glucose transport and GLUT-4. Am J Physiol Endocrinol Metab 264: E583-E593, 1993.

299. Meshulam T, Simard JR, Wharton J, Hamilton JA, Pilch PF. Role of caveolin-1 and cholesterol in transmembrane fatty acid movement. Biochemistry 45: 2882-2893, 2006.

300. Milger K, Herrmann T, Becker C, Gotthardt D, Zickwolf J, Ehehalt R, Watkins PA, Stremmel W, Fullekrug J. Cellular uptake of fatty acids driven by the ER-localized acyl-CoA synthetase FATP4. J Cell Sci 119: 4678-4688, 2006.

301. Miller AM, Brestoff JR, Phelps CB, Berk EZ, Reynolds TH 4th. Rapamycin does not improve insulin sensitivity despite elevated mammalian target of rapamycin complex 1 activity in muscles of ob/ob mice. Am J Physiol Regul Integr Comp Physiol 295: R1431-R1438, 2008

302. Minokoshi Y, Kim YB, Peroni OD, Fryer LG, Muller C, Carling D, Kahn BB. Leptin stimulates fatty-acid oxidation by activating AMP-activated protein kinase. Nature 415: 339-343, 2002

303. Mitra SK, Schlaepfer DD. Integrin-regulated FAK-Src signaling in normal and cancer cells. Curr Opin Cell Biol 18: 516-523, 2006.

304. Mogensen M, Sahlin K, Fernstrom M, Glintborg D, Vind BF, Beck-Nielsen H, Hojlund $\mathbf{K}$. Mitochondrial respiration is decreased in skeletal muscle of patients with type 2 diabetes. Diabetes 56: 1592-1599, 2007.

305. Momken I, Chabowski A, Bonen A. Effect of leptin on fatty acid metabolism and subcellular distribution of FAT/CD36 in cardiomyocytes. (Abstract). Northern Lights Conference. Canadian Federation of Biological Societies 2nd, Guelph, Ontario, Canada, 2005.

306. Mora A, Sakamoto K, McManus EJ, Alessi DR. Role of the PDK1-PKB-GSK3 pathway in regulating glycogen synthase and glucose uptake in the heart. FEBS Lett 579: 3632-3638, 2005.

307. Motojima K, Passilly P, Peters JM, Gonzalez FJ, Latruffe N. Expression of putative fatty acid transporter genes are regulated by peroxisome proliferator-activated receptor alpha and gamma activators in a tissue- and inducer-specific manner. J Biol Chem 273: 16710-16714, 1998.

308. Muller H, Deckers K, Eckel J. The fatty acid translocase (FAT)/ CD36 and the glucose transporter GLUT4 are localized in different cellular compartments in rat cardiac muscle. Biochem Biophys Res Commun 293: 665-669, 2002.

309. Muoio DM, Dohm GL, Fiedorek FT, Tapscott EB Jr, Coleman RA. Leptin directly alters lipid partitioning in skeletal muscle. Diabetes 46: 1360-1363, 1997.

310. Muoio DM, Dohm GL, Tapscott EB, Coleman RA. Leptin opposes insulin's effects on fatty acid partitioning in muscles isolated from obese ob/ob mice. Am J Physiol Endocrinol Metab 276: E913E921, 1999.

311. Nakamura T, Sugihara H, Inaba T, Kinoshita N, Adachi Y, Hirasaki S, Matsuo A, Azuma A, Nakagawa M. CD36 deficiency has little influence on the pathophysiology of hypertrophic cardiomyopathy. J Mol Cell Cardiol 31: 1253-1259, 1999.
312. Nawrocki A, Gorska M, Zendzian-Piotrowska M, Gorski J. Effect of acute streptozotocin diabetes on fatty acid content and composition in different lipid fractions of rat skeletal muscle. Horm Metab Res 31: 252-256, 1999.

313. Nicholson AC, Febbraio M, Han J, Silverstein RL, Hajjar DP. CD36 in atherosclerosis. The role of a class B macrophage scavenger receptor. Ann NY Acad Sci 902: 128-131, 2000.

314. Nickerson J, Bonen A. Defining a role for skeletal muscle fatty acid transport proteins. Northern Lights Conference. Canadian Federation of Biological Societies 2nd Guelph, Ontario, Canada 2005, p. F47.

315. Nickerson JG, Alkhateeb H, Benton CR, Lally J, Nickerson J, Han XX, Wilson MH, Jain SS, Snook LA, Glatz JFC, Chabowski A, Luiken JJFP, Bonen A. Greater transport efficiencies of the membrane fatty acid transporters FAT/CD36 and FATP4 compared with FABPpm and FATP1: differential effects on fatty acid esterification and oxidation in rat skeletal muscle. J Biol Chem 284: 16522-16530, 2009.

316. Nishimura H, Pallardo FV, Seidner GA, Vannucci S, Simpson IA, Birnbaum MJ. Kinetics of GLUT1 and GLUT4 glucose transporters expressed in Xenopus oocytes. J Biol Chem 268: 85148520, 1993.

317. Odland LM, Heigenhauser GJ, Lopaschuk GD, Spriet LL. Human skeletal muscle malonyl-CoA at rest and during prolonged submaximal exercise. Am J Physiol Endocrinol Metab 270: E541E544, 1996.

318. Odland LM, Howlett RA, Heigenhauser GJ, Hultman E, Spriet LL. Skeletal muscle malonyl-CoA content at the onset of exercise at varying power outputs in humans. Am J Physiol Endocrinol Metab 274: E1080-E1085, 1998.

319. Oh E, Spurlin BA, Pessin JE, Thurmond DC. Munc18c heterozygous knockout mice display increased susceptibility for severe glucose intolerance. Diabetes 54: 638-647, 2005.

320. Okamoto F, Tanaka T, Sohmiya K, Kawamura K. CD36 abnormality and impaired myocardial long-chain fatty acid uptake in patients with hypertrophic cardiomyopathy. Jpn Circ $J$ 62: $499-$ 504, 1998.

321. Ouwens DM, Boer C, Fodor M, de Galan P, Heine RJ, Maassen JA, Diamant M. Cardiac dysfunction induced by high-fat diet is associated with altered myocardial insulin signalling in rats. Diabetologia 48: 1229-1237, 2005.

322. Ouwens DM, Diamant M, Foddor M, Habets D, Pelsers MMAL, El Hanaoui M, Dang ZC, van den Brom CE, Vlasblom R, Rietdijk A, Boer C, Coort SML, Glatz JFC, Luiken JJFP. Cardiac contractile dysfunction in insulin-resistant rats fed a high fat diet is associated with elevated CD36-mediated fatty acid uptake and esterification. Diabetologia 50: 1938-1948, 2007.

323. Overath P, Pauli G, Schairer HU. Fatty acid degradation in Escherichia coli. An inducible acyl-CoA synthetase, the mapping of old-mutations, and the isolation of regulatory mutants. Eur J Biochem 7: 559-574, 1969 .

324. Palanivel R, Eguchi M, Shuralyova I, Coe I, Sweeney G. Distinct effects of short- and long-term leptin treatment on glucose and fatty acid uptake and metabolism in HL-1 cardiomyocytes. Metabolism 55: 1067-1075, 2006.

325. Palanivel R, Sweeney G. Regulation of fatty acid uptake and metabolism in L6 skeletal muscle cells by resistin. FEBS Lett 579: 5049-5054, 2005.

326. Pan DA, Lillioja S, Kriketos AD, Milner MR, Baur LA, Jenkins AB, Storlien LH. Skeletal muscle triglyceride levels are inversely related to insulin action. Diabetes 46: 983-988, 1997.

327. Parton RG, Simons K. The multiple faces of caveolae. Nat Rev Mol Cell Biol 8: 185-194, 2007.

328. Pei Z, Fraisl P, Berger J, Jia Z, Forss-Petter S, Watkins PA. Mouse very long-chain acyl-CoA synthetase $3 /$ fatty acid transport protein 3 catalyzes fatty acid activation but not fatty acid transport in MA-10 cells. $J$ Biol Chem 279: 54454-54462, 2004.

329. Pelsers MM, Lutgerink JT, van Nieuwenhoven FA, Tandon NN, van der Vusse GJ, Arends JW, Hoogenboom HR, Glatz JFC. A sensitive immunoassay for rat fatty acid translocase (CD36) using phage antibodies selected on cell transfectants: abundant presence of fatty acid translocase/CD36 in cardiac and red skeletal 
muscle and up-regulation in diabetes. Biochem $J$ 337: 407-414, 1999.

330. Pelsers MM, Tsintzas K, Boon H, Jewell K, Norton L, Luiken JJ, Glatz JF, van Loon LJ. Skeletal muscle fatty acid transporter protein expression in type 2 diabetes patients compared with overweight, sedentary men and age-matched, endurance-trained cyclists. Acta Physiol 190: 209-219, 2007.

331. Petersen KF, Dufour S, Befroy D, Garcia R, Shulman GI. Impaired mitochondrial activity in the insulin-resistant offspring of patients with type 2 diabetes. $N$ Engl $J$ Med 350: 664-671, 2004.

332. Ploug T, Wojtaszewski J, Kristiansen S, Hespel P, Galbo H, Richter E. Glucose transport and transporters in muscle giant vesicles: differential effects of insulin and contractions. Am J Physiol Endocrinol Metab 264: E270-E278, 1993.

333. Pohl J, Ring A, Ehehalt R, Schulze-Bergkamen H, Schad A, Verkade P, Stremmel W. Long-chain fatty acid uptake into adipocytes depends on lipid raft function. Biochemistry 43: 41794187, 2004.

334. Pohl J, Ring A, Korkmaz U, Ehehalt R, Stremmel W. FAT/ CD36-mediated long-chain fatty acid uptake in adipocytes requires plasma membrane rafts. Mol Biol Cell 16: 24-31, 2005.

335. Pohl J, Ring A, Stremmel W. Uptake of long-chain fatty acids in HepG2 cells involves caveolae: analysis of a novel pathway. J Lipid Res 43: 1390-1399, 2002

336. Potter BJ, Stump D, Schwieterman W, Sorrentino D, Jacobs LN, Kiang CL, Rand JH, Berk PD. Isolation and partial characterization of plasma membrane fatty acid binding proteins from myocardium and adipose tissue and their relationship to analogous proteins in liver and gut. Biochem Biophys Res Commun 148: 1370-1376, 1987.

337. Pravenec M, Landa V, Zidek V, Musilova M, Kren V, Kazdova L, Aitman TJ, Glazier A, Ibrahimi A, Abumrad NA, Qi N, Wang JM, St. Lezin EM, Kurtz TW. Transgenic rescue of defective Cd36 ameliorates insulin resistance in spontaneously hypertensive rats. Nature Genet 27: 156-158, 2001.

338. Pravenec M, Zidek V, Simakova M, Kren V, Krenova D, Horky K, Jachymova M, Kazdova L, Aitman T, Churchil P, Webb RC, Hingarh NH, Yang Y, Wang JM, St. Lezin EM, Kurtz TW. Genetics of Cd36 and the clustering of multiple cardiovascular risk factors in spontaneous hypertension. J Clin Invest 109: 1651-1657, 1999.

339. Prekeris R, Klumperman J, Scheller RH. A Rab11/Rip11 protein complex regulates apical membrane trafficking via recycling endosomes. Mol Cell 6: 1437-1448, 2000.

340. Qi N, Kazdova L, Zidek V, Landa V, Kren V, Pershadsingh HA, Lezin ES, Abumrad NA, Pravenec M, Kurtz TW. Pharmacogenetic evidence that cd36 is a key determinant of the metabolic effects of pioglitazone. J Biol Chem 277: 48501-48507, 2002.

341. Qiao L, Zou C, Shao P, Schaack J, Johnson PF, Shao J. Transcriptional regulation of fatty acid translocase/CD36 expression by CCAAT/enhancer-binding protein alpha. $J$ Biol Chem 283: 87888795, 2008

342. Rabol R, Boushel R, Dela F. Mitochondrial oxidative function and type 2 diabetes. Appl Physiol Nutr Metab 31: 675-683, 2006.

343. Raney MA, Turcotte LP. Evidence for the involvement of CaMKII and AMPK in $\mathrm{Ca}^{2+}$-dependent signaling pathways regulating FA uptake and oxidation in contracting rodent muscle. $J$ Appl Physiol 104: 1366-1373, 2008.

344. Raney MA, Turcotte LP. Regulation of contraction-induced FA uptake and oxidation by AMPK and ERK1/2 is intensity dependent in rodent muscle. Am J Physiol Endocrinol Metab 291: E1220E1227, 2006

345. Raney MA, Yee AJ, Todd MK, Turcotte LP. AMPK activation is not critical in the regulation of muscle FA uptake and oxidation during low-intensity muscle contraction. Am J Physiol Endocrinol Metab 288: E592-E598, 2005.

346. Richards MR, Harp JD, Ory DS, Schaffer JE. Fatty acid transport protein 1 and long-chain acyl coenzyme A synthetase 1 interact in adipocytes. J Lipid Res 47: 665-672, 2006.

347. Richards MR, Listenberger LL, Kelly AA, Lewis SE, Ory D, Schaffer JE. Oligomerization of the murine fatty acid transport protein 1. J Biol Chem 278: 10477-10483, 2003.
348. Richieri GV, Anel A, Kleinfeld A. Interactions of long-chain fatty acids and albumin: determination of free fatty acid levels using the fluorescent probe ADIFAB. Biochemistry 32: 7574-7580, 1993.

349. Richieri GV, Kleinfeld AM. Unbound free fatty acid levels in human serum. J Lipid Res 36: 229-240, 1995.

350. Richieri GV, Ogata RT, Kleinfeld A. Equilibrium constants for the binding of fatty acids with fatty acid-binding proteins from adipocyte, intestine, heart, and liver measured with the fluorescent probe ADIFAB. J Biol Chem 269: 23918-23930, 1994.

351. Richieri GV, Ogata RT, Kleinfeld AM. A fluorescently labeled intestinal fatty acid binding protein. Interactions with fatty acids and its use in monitoring free fatty acids. J Biol Chem 267: 2349523501, 1992.

352. Richter EA, Nielsen JN, Jørgensen SB, Frosig C, Birk JB, Wojtaszewski JF. Exercise signalling to glucose transport in skeletal muscle. Proc Nutr Soc 63: 211-216, 2004.

353. Richter EA, Nielsen JN, Jørgensen SB, Frosig C, Wojtaszewski JF. Signalling to glucose transport in skeletal muscle during exercise. Acta Physiol Scand 178: 329-335, 2003.

354. Ring A, Le Lay S, Pohl J, Verkade P, Stremmel W. Caveolin-1 is required for fatty acid translocase (FAT/CD36) localization and function at the plasma membrane of mouse embryonic fibroblasts. Biochim Biophys Acta 1761: 416-423, 2006.

355. Ritov VB, Menshikova EV, He J, Ferrell RE, Goodpaster BH, Kelley DE. Deficiency of subsarcolemmal mitochondria in obesity and type 2 diabetes. Diabetes 54: 8-14, 2005.

356. Roepstorff C, Halberg N, Hillig T, Saha AK, Ruderman NB, Wojtaszewski JF, Richter EA, Kiens B. Malonyl-CoA and carnitine in regulation of fat oxidation in human skeletal muscle during exercise. Am J Physiol Endocrinol Metab 288: E133-E142, 2005.

357. Roepstorff C, Thiele M, Hillig T, Pilegaard H, Richter EA, Wojtaszewski JF, Kiens B. Higher skeletal muscle alpha2AMPK activation and lower energy charge and fat oxidation in men than in women during submaximal exercise. J Physiol 574: 125-138, 2006.

358. Rose H, Hennecke T, Kammermeier H. Sarcolemmal fatty acid transfer in isolated cardiomyocytes governed by albumin $/ \mathrm{mem}$ brane-lipid partition. J Mol Cell Cardiol 22: 883-892, 1990.

359. Rosenblatt-Velin N, Montessuit C, Papageorgiou I, Terrand J, Lerch R. Postinfarction heart failure in rats is associated with upregulation of GLUT-1 and downregulation of genes of fatty acid metabolism. Cardiovasc Res 52: 407-416, 2001.

360. Rupp H, Elimban V, Dhalla NS. Modification of subcellular organelles in pressure-overloaded heart by etomoxir, a carnitine palmitoyltransferase I inhibitor. FASEB $J$ 6: 2349-2353, 1992.

361. Ryder JW, Yang J, Galuska D, Rincon J, Bjornholm M, Krook A, Lund S, Pedersen O, Wallberg-Henriksson H, Zierath JR, Holman GD. Use of a novel impermeable biotinylated photolabeling reagent to assess insulin- and hypoxia-stimulated cell surface GLUT4 content in skeletal muscle from type 2 diabetic patients. Diabetes 49: 647-654, 2000.

362. Sakamoto K, Goodyear LJ. Invited review: intracellular signaling in contracting skeletal muscle. J Appl Physiol 93: 369-383, 2002.

363. Sakamoto K, Goransson O, Hardie DG, Alessi DR. Activity of LKB1 and AMPK-related kinases in skeletal muscle: effects of contraction, phenformin, and AICAR. Am J Physiol Endocrinol Metab 287: E310-E317, 2004.

364. Sakamoto K, McCarthy A, Smith D, Green KA, Grahame Hardie D, Ashworth A, Alessi DR. Deficiency of LKB1 in skeletal muscle prevents AMPK activation and glucose uptake during contraction. EMBO J 24: 1810-1820, 2005.

365. Saltiel AR, Kahn CR. Insulin signalling and the regulation of glucose and lipid metabolism. Nature 414: 799-806, 2001.

366. Sandoval A, Fraisl P, Arias-Barrau E, Dirusso CC, Singer D, Sealls W, Black PN. Fatty acid transport and activation and the expression patterns of genes involved in fatty acid trafficking. Arch Biochem Biophys 477: 363-371, 2008.

367. Sano H, Kane S, Sano E, Miinea CP, Asara JM, Lane WS, Garner CW, Lienhard GE. Insulin-stimulated phosphorylation of a Rab GTPase-activating protein regulates GLUT4 translocation. J Biol Chem 278: 14599-14602, 2003.

368. Sasaoka T, Hori H, Wada T, Ishiki M, Haruta T, Ishihara H, Kobayashi M. SH2-containing inositol phosphatase 2 negatively 
regulates insulin-induced glycogen synthesis in L6 myotubes. Diabetologia 44: 1258-1267, 2001.

369. Sato O, Kuriki C, Fukui Y, Motojima K. Dual promoter structure of mouse and human fatty acid translocase/CD36 genes and unique transcriptional activation by peroxisome proliferator-activated receptor alpha and gamma ligands. J Biol Chem 277: 15703-15711, 2002.

370. Schaap FG, Binas B, Danneberg H, van der Vusse GJ, Glatz JF. Impaired long-chain fatty acid utilization by cardiac myocytes isolated from mice lacking the heart-type fatty acid binding protein gene. Circ Res 85: 329-337, 1999.

371. Schaffer JE, Lodish HF. Expression cloning and characterization of a novel adipocyte long chain fatty acid transport protein. Cell 79: 427-436, 1994.

372. Schenk S, Horowitz JF. Coimmunoprecipitation of FAT/CD36 and CPTI in skeletal muscle increases proportionally with fat oxidation after endurance exercise training. Am J Physiol Endocrinol Metab 291: E254-E260, 2006.

373. Schmelter T, Trigatti BL, Gerber GE, Mangroo D. Biochemical demonstration of the involvement of fatty acyl-CoA synthetase in fatty acid translocation across the plasma membrane. J Biol Chem 279: 24163-24170, 2004.

374. Schmitt B, Fluck M, Decombaz J, Kreis R, Boesch C, Wittwer M, Graber F, Vogt M, Howald H, Hoppeler H. Transcriptional adaptations of lipid metabolism in tibialis anterior muscle of endurance-trained athletes. Physiol Genomics 15: 148-157, 2003.

375. Schmuth M, Ortegon AM, Mao-Qiang M, Elias PM, Feingold KR, Stahl A. Differential expression of fatty acid transport proteins in epidermis and skin appendages. J Invest Dermatol 125: 1174-1181, 2005.

376. Schwenk RW, Luiken JJ, Bonen A, Glatz JF. Regulation of sarcolemmal glucose and fatty acid transporters in cardiac disease. Cardiovasc Res 79: 249-258, 2008.

377. Schwenk RW, Luiken JJ, Eckel J. FIP2 and Rip11 specify Rab11a-mediated cellular distribution of GLUT4 and FAT/CD36 in H9c2-hIR cells. Biochem Biophys Res Commun 363: 119-125, 2007.

378. Schwieterman W, Sorrentino D, Potter BJ, Rand J, Kiang CL, Stump D, Berk PD. Uptake of oleate by isolated rat adipocytes is mediated by a $40-\mathrm{kDa}$ plasma membrane fatty acid binding protein closely related to that in liver and gut. Proc Natl Acad Sci USA 85: 359-363, 1988.

379. Sebastian D, Guitart M, Garcia-Martinez C, Mauvezin C, Orellana-Gavalda JM, Serra D, Gomez-Foix AM, Hegardt FG, Asins G. Novel role of FATP1 in mitochondrial fatty acid oxidation in skeletal muscle cells. J Lipid Res. In press.

380. Sharma S, Adrogue JV, Golfman L, Uray I, Lemm J, Youker K, Noon GP, Frazier OH, Taegtmeyer H. Intramyocardial lipid accumulation in the failing human heart resembles the lipotoxic rat heart. FASEB J 18: 1692-1700, 2004.

381. Shepherd PR. Mechanisms regulating phosphoinositide 3-kinase signalling in insulin-sensitive tissues. Acta Physiol Scand 183: 3-12, 2005.

382. Simard JR, Meshulam T, Pillai BK, Kirber MT, Brunaldi K, Xu S, Pilch PF, Hamilton JA. Caveolins sequester fatty acids on the cytoplasmic leaflet of the plasma membrane, augment triglyceride formation and protect cells from lipotoxicity. J Lipid Res. In press.

383. Simard JR, Pillai BK, Hamilton JA. Fatty acid flip-flop in a model membrane is faster than desorption into the aqueous phase. Biochemistry 47: 9081-9089, 2008.

384. Simoneau JA, Veerkamp JH, Turcotte LP, Kelley DE. Markers of capacity to utilize fatty acids in human skeletal muscle: relation to insulin resistance and obesity and effects of weight loss. FASEB $J$ 13: 2051-2060, 1999.

385. Smith AC, Bruce CR, Dyck DJ. AMP kinase activation with AICAR further increases fatty acid oxidation and blunts triacylglycerol hydrolysis in contracting rat soleus muscle. J Physiol 565: 547-553, 2005.

386. Smith AC, Bruce CR, Dyck DJ. AMP kinase activation with AICAR simultaneously increases fatty acid and glucose oxidation in resting rat soleus muscle. J Physiol 565: 537-546, 2005.

387. Smith AC, Mullen KL, Junkin KA, Nickerson J, Chabowski A, Bonen A, Dyck DJ. Metformin and exercise reduce muscle FAT/ CD36 and lipid accumulation and blunt theprogression of high-fat diet induced hyperglycemia. Am J Physiol Endocrinol Metab 293: E172-E181, 2007.

388. Smith J, Su X, El-Maghrabi R, Stahl PD, Abumrad NA. Opposite regulation of CD36 ubiquitination by fatty acids and insulin: effects on fatty acid uptake. J Biol Chem 283: 13578-13585, 2008.

389. Soeters MR, Sauerwein HP, Groener JE, Aerts JM, Ackermans MT, Glatz JF, Fliers E, Serlie MJ. Gender-related differences in the metabolic response to fasting. $J$ Clin Endocrinol Metab 92: 3646-3652, 2007.

390. Sogaard M, Tani K, Ye RR, Geromanos S, Tempst P, Kirchhausen T, Rothman JE, Sollner T. A rab protein is required for the assembly of SNARE complexes in the docking of transport vesicles. Cell 78: 937-948, 1994.

391. Song XM, Ryder JW, Kawano Y, Chibalin A, Krook A, Zierath JR. Muscle fiber type specificity in insulin signal transduction. Am J Physiol Regul Integr Comp Physiol 277: R1690-R1696, 1999.

392. Sonnichsen B, De Renzis S, Nielsen E, Rietdorf J, Zerial M. Distinct membrane domains on endosomes in the recycling pathway visualized by multicolor imaging of Rab4, Rab5, and Rab11. J Cell Biol 149: 901-914, 2000.

393. Sorrentino D, Stump D, Potter BJ, Robinson RB, White R, Kiang CL, Berk PD. Oleate uptake by cardiac myocytes is carrier mediated and involves a 40-kDa plasma membrane fatty acid binding protein similar to that in liver, adipose tissue, and gut. J Clin Invest 82: 928-935, 1988

394. Sorrentino D, Van Ness K, Moukabary K, Berk PD. Hepatocellular ${ }^{22} \mathrm{Na}^{+}$uptake: effect of oleate. Am J Physiol Gastrointest Liver Physiol 261: G1024-G1029, 1991.

395. Spector AA, John K, Fletcher JE. Binding of long-chain fatty acids to bovine serum albumin. J Lipid Res 10: 56-67, 1969.

396. Spitsberg VL, Matitashvili E, Gorewit RC. Association and coexpression of fatty-acid-binding protein and glycoprotein CD36 in the bovine mammary gland. Eur J Biochem 230: 872-878, 1995.

397. St-Denis JF, Cushman SW. Role of SNARE's in the GLUT4 translocation response to insulin in adipose cells and muscle. $J$ Basic Clin Physiol Pharmacol 9: 153-165, 1998.

398. Staels B, Fruchart JC. Therapeutic roles of peroxisome proliferator-activated receptor agonists. Diabetes 54: 2460-2470, 2005.

399. Stahl A, Evans JG, Pattel S, Hirsch D, Lodish HF. Insulin causes fatty acid transport protein translocation and enhanced fatty acid uptake in adipocytes. Dev Cell 2: 477-488, 2002.

400. Stanley WC, Recchia FA, Lopaschuk GD. Myocardial substrate metabolism in the normal and failing heart. Physiol Rev 85: 10931129, 2005.

401. Stapleton D, Mitchelhill KI, Gao G, Widmer J, Michell BJ, Teh T, House CM, Fernandez CS, Cox T, Witters LA, Kemp BE. Mammalian AMP-activated protein kinase subfamily. J Biol Chem 271: 611-614, 1996.

402. Starritt EC, Howlett RA, Heigenhauser GJ, Spriet LL. Sensitivity of CPT I to malonyl-CoA in trained and untrained human skeletal muscle. Am J Physiol Endocrinol Metab 278: E462-E468, 2000.

403. Steinberg GR, Dyck DJ. Development of leptin resistance in rat soleus muscle in response to high fat diets. Am J Physol Endocrinol Metab 279: E1374-E1382, 2000.

404. Steinberg GR, Dyck DJ, Calles-Escandon J, Tandon NN, Luiken JJFP, Glatz JF, Bonen A. Chronic leptin administration decreases fatty acid uptake and fatty acid transporters in rat skeletal muscle. J Biol Chem 277: 8854-8860, 2002.

405. Steinberg GR, Parolin ML, Heigenhauser GJF, Dyck DJ. Leptin increases fatty acid oxidation in lean but not obese human skeletal muscle: evidence of peripheral leptin resistance. Am $J$ Physiol Endocrinol Metab 283: E187-E192, 2002.

406. Steinberg GR, Rush JW, Dyck DJ. AMPK expression and phosphorylation are increased in rodent muscle after chronic leptin treatment. Am J Physiol Endocrinol Metab 284: E648-E654, 2003.

407. Storch J, Corsico B. The emerging functions and mechanisms of mammalian fatty acid-binding proteins. Annu Rev Nutr 28: 73-95, 2008.

408. Storch J, Kleinfeld AM. Transfer of long-chain fluorescent free fatty acids between unilamellar vesicles. Biochemistry 25: 1717$1726,1986$. 
409. Storlien LH, Jenkins AB, Chisholm DJ, Pascoe WS, Khouri S, Kraegen EW. Influence of dietary fat composition on development of insulin resistance in rats. Diabetes 40: 280-289, 1991.

410. Stratford S, Hoehn KL, Liu F, Summers SA. Regulation of insulin action by ceramide: dual mechanisms linking ceramide accumulation to the inhibition of Akt/protein kinase B. J Biol Chem 279: 36608-36615, 2004.

411. Stremmel W. Fatty acid uptake by isolated heart myocytes represents a carrier-mediated transport process. J Clin Invest 81: 844852, 1988.

412. Stremmel W, Berk PD. Hepatocellular influx of $\left[{ }^{14} \mathrm{C}\right]$ oleate reflects membrane transport rather than intracellular metabolism or binding. Proc Natl Acad Sci USA 83: 3086-3090, 1986.

413. Stremmel W, Berk PD. Hepatocellular uptake of sulfobromophthalein and bilirubin is selectively inhibited by an antibody to the liver plasma membrane sulfobromophthalein/bilirubin binding protein. J Clin Invest 78: 822-826, 1986.

414. Stremmel W, Lotz G, Strohmeyer G, Berk PD. Identification, isolation, and partial characterization of a fatty acid binding protein from rat jejunal microvillous membranes. J Clin Invest 75: 10681076,1985

415. Stremmel W, Strohmeyer G, Berk PD. Hepatocellular uptake of oleate is energy dependent, sodium linked, and inhibited by an antibody to a hepatocyte plasma membrane fatty acid binding protein. Proc Natl Acad Sci USA 83: 3584-3588, 1986.

416. Stremmel W, Strohmeyer G, Borchard F, Kochwa S, Berk PD. Isolation and partial characterization of a fatty acid binding protein in rat liver plasma membranes. Proc Natl Acad Sci USA 82: 4-8, 1985.

417. Stuart LM, Bell SA, Stewart CR, Silver JM, Richard J, Goss JL, Tseng AA, Zhang A, El Khoury JB, Moore KJ. CD36 signals to the actin cytoskeleton and regulates microglial migration via a p130Cas complex. J Biol Chem 282: 27392-27401, 2007.

418. Stuhlsatz-Krouper SM, Bennett NE, Schaffer JE. Substitution of alanine for serine 250 in the murine fatty acid transport protein inhibits long chain fatty acid transport. J Biol Chem 273: 2864228650, 1998.

419. Stump DD, Zhou SL, Berk PD. Comparison of plasma membrane FABP and mitochondrial isoform of aspartate aminotransferase from rat liver. Am J Physiol Gastrointest Liver Physiol 265: G894G902, 1993.

420. Su X, Abumrad NA. Cellular fatty acid uptake: a pathway under construction. Trends Endocrinol Metab 20: 72-77, 2009.

421. Summers SA, Garza LA, Zhou H, Birnbaum MJ. Regulation of insulin-stimulated glucose transporter GLUT4 translocation and Akt kinase activity by ceramide. Mol Cell Biol 18: 5457-5464, 1998.

422. Taegtmeyer H. Metabolism-the lost child of cardiology. J Am Coll Cardiol 36: 1386-1388, 2000.

423. Talanian JL, Galloway SD, Heigenhauser GJ, Bonen A, Spriet LL. Two weeks of high-intensity aerobic interval training increases the capacity for fat oxidation during exercise in women. $J$ Appl Physiol 102: 1439-1447, 2007.

424. Tanaka T, Nakata T, Oka T, Ogawa T, Okamoto F, Kusaka Y, Sohmiya K, Shimamoto K, Itakura K. Defect in human myocardial long-chain fatty acid uptake is caused by FAT/CD36 mutations. J Lipid Res 42: 751-759, 2001.

425. Tanaka T, Sohmiya K, Kawamura K. Is CD36 deficiency an etiology of hereditary hypertrophic cardiomyopathy? J Mol Cell Cardiol 29: 121-127, 1997.

426. Tang X, Powelka AM, Soriano NA, Czech MP, Guilherme A. PTEN, but not SHIP2, suppresses insulin signaling through the phosphatidylinositol 3-kinase/Akt pathway in 3T3-L1 adipocytes. J Biol Chem 280: 22523-22529, 2005.

427. Tao N, Wagner SJ, Lublin DM. CD36 is palmitolylated on both Nand C-terminal cytoplasmic tails. J Biol Chem 271: 22315-22320, 1996.

428. Thong FS, Dugani CB, Klip A. Turning signals on and off: GLUT4 traffic in the insulin-signaling highway. Physiology 20: 271-284, 2005.

429. Thurmond DC, Ceresa BP, Okada S, Elmendorf JS, Coker K, Pessin JE. Regulation of insulin-stimulated GLUT4 translocation by Munc18c in 3T3L1 adipocytes. J Biol Chem 273: 33876-33883, 1998.
430. Thyfault JP, Kraus RM, Hickner RC, Howell AW, Wolfe RR, Dohm GL. Impaired plasma fatty acid oxidation in extremely obese women. Am J Physiol Endocrinol Metab 287: E1076-E1081, 2004.

431. Tomas E, Sevilla L, Palacin M, Zorzano A. The insulin-sensitive GLUT4 storage compartment is a postendocytic and heterogeneous population recruited by acute exercise. Biochem Biophys Res Commun 284: 490-495, 2001.

432. Tong F, Black PN, Coleman RA, DiRusso CC. Fatty acid transport by vectorial acylation in mammals: roles played by different isoforms of rat long-chain acyl-CoA synthetases. Arch Biochem Biophys 447: 46-52, 2006.

433. Tontonoz P, Nagy L, Alvarez JG, Thomazy VA, Evans RM. PPARgamma promotes monocyte/macrophage differentiation and uptake of oxidized LDL. Cell 93: 241-252, 1998.

434. Towler MC, Hardie DG. AMP-activated protein kinase in metabolic control and insulin signaling. Circ Res 100: 328-341, 2007.

435. Treebak JT, Glund S, Deshmukh A, Klein DK, Long YC, Jensen TE, Jørgensen SB, Viollet B, Andersson L, Neumann D, Wallimann T, Richter EA, Chibalin AV, Zierath JR, Wojtaszewski JF. AMPK-mediated AS160 phosphorylation in skeletal muscle is dependent on AMPK catalytic and regulatory subunits. Diabetes 55: 2051-2058, 2006.

436. Trigatti BL, Anderson RG, Gerber GE. Identification of caveolin-1 as a fatty acid binding protein. Biochem Biophys Res Commun 255: 34-39, 1999.

437. Trigatti BL, Baker AD, Rajaratnam K, Rachubinski RA, Gerber GE. Fatty acid uptake in Candida tropicalis: induction of a saturable process. Biochem Cell Biol 70: 76-80, 1992.

438. Trigatti BL, Mangroo D, Gerber GE. Photoaffinity labeling and fatty acid permeation in 3T3-L1 adipocytes. J Biol Chem 266: 22621-22625, 1991.

439. Trischler M, Stoorvogel W, Ullrich O. Biochemical analysis of distinct Rab5- and Rab11-positive endosomes along the transferrin pathway. J Cell Sci 112: 4773-4783, 1999.

440. Tsiani E, Bogdanovic E, Sorisky A, Nagy L, Fantus IG. Tyrosine phosphatase inhibitors, vanadate and pervanadate, stimulate glucose transport and GLUT translocation in muscle cells by a mechanism independent of phosphatidylinositol 3-kinase and protein kinase C. Diabetes 47: 1676-1686, 1998.

441. Tucker MZ, Turcotte LP. Impaired fatty acid oxidation in muscle of aging rats perfused under basal conditions. Am J Physiol Endocrinol Metab 282: E1102-E1109, 2002.

442. Tunstall RJ, Mehan KA, Wadley GD, Collier GR, Bonen A, Hargreaves M, Cameron-Smith D. Exercise training increases lipid metabolism gene expression in human skeletal muscle. Am J Physiol Endocrinol Metab 283: E66-E72, 2002.

443. Turcotte LP, Raney MA, Todd MK. ERK1/2 inhibition prevents contraction-induced increase in plasma membrane FAT/CD36 content and FA uptake in rodent muscle. Acta Physiol Scand 184: 131-139, 2005.

444. Turcotte LP, Srivastava AK, Chiasson JL. Fasting increases plasma membrane fatty acid binding $\left(\mathrm{FABP}_{\mathrm{pm}}\right)$ in red skeletal muscle. Mol Cell Biochem 166: 153-158, 1997.

445. Turcotte LP, Swenberger JR, Tucker MZ, Trump G, Yee AJ, Luiken JJFP, Bonen A. Muscle palmitate transport and binding are saturable and inhibited by antibodies to FABPpm. Mol Cell Biochem 210: 53-63, 2000.

446. Turcotte LP, Swenberger JR, Tucker MZ, Yee AJ. Increased fatty acid uptake and altered fatty acid metabolism in insulinresistant muscle of obese Zucker rats. Diabetes 50: 1389-1396, 2001.

447. Turcotte LP, Swenberger JR, Tucker MZ, Yee AJ. Traininginduced elevation in FABPpm is associated with increased palmitate use in contracting muscle. J Appl Physiol 87: 285-293, 1999.

448. Uchiyama A, Aoyama T, Kamijo K, Uchida Y, Kondo N, Orii T, Hashimoto T. Molecular cloning of cDNA encoding rat very longchain acyl-CoA synthetase. J Biol Chem 271: 30360-30365, 1996.

449. Uhlig M, Passlack W, Eckel J. Functional role of Rab11 in GLUT4 trafficking in cardiomyocytes. Mol Cell Endocrinol 235: 1-9, 2005.

450. Ullrich O, Reinsch S, Urbe S, Zerial M, Parton RG. Rab11 regulates recycling through the pericentriolar recycling endosome. J Cell Biol 135: 913-924, 1996. 
451. Unger RH. Lipotoxic diseases. Annu Rev Med 53: 319-336, 2002.

452. Uphues I, Kolter T, Goud B, Eckel J. Insulin-induced translocation of the glucose transporter GLUT4 in cardiac muscle: studies on the role of small-molecular-mass GTP-binding proteins. Biochem J 301: 177-182, 1994.

453. Van der Heyden MA, Wijnhoven TJ, Opthof T. Molecular aspects of adrenergic modulation of cardiac L-type $\mathrm{Ca}^{2+}$ channels. Cardiovasc Res 65: 28-39, 2005.

454. Van der Lee KA, Vork MM, De Vries JE, Willemsen PH, Glatz JF, Reneman RS, van der Vusse GJ, van Bilsen M. Long-chain fatty acid-induced changes in gene expression in neonatal cardiac myocytes. J Lipid Res 41: 41-47, 2000.

455. Van der Lee KA, Willemsen PH, Samec S, Seydoux J, Dulloo AG, Pelsers MM, Glatz JF, van der Vusse GJ, van Bilsen M. Fasting-induced changes in the expression of genes controlling substrate metabolism in the rat heart. J Lipid Res 42: 1752-1758, 2001.

456. Van der Vusse GJ, Roemen THM. Gradient of fatty acids from blood plasma to skeletal muscle in dogs. J Appl Physiol 78: 18391843,1995

457. Van Loon LJ, Koopman R, Manders R, van der Weegen W, van Kranenburg GP, Keizer HA. Intramyocellular lipid content in type 2 diabetes patients compared with overweight sedentary men and highly trained endurance athletes. Am J Physiol Endocrinol Metab 287: E558-E565, 2004.

458. Van Nieuwenhoven FA, Luiken JJFP, de Jong YF, Grimaldi PA, van der Vusse GJ, Glatz JFC. Stable transfection of fatty acid translocase (CD36) in a rat heart muscle cell-line (H9c2 ). $J$ Lipid Res 39: 2039-2047, 1998.

459. Van Nieuwenhoven FA, Willemsen PH, van der Vusse GJ, Glatz JFC. Co-expression in rat heart and skeletal mucle of four genes coding for proteins implicated in long-chain fatty acid uptake. Int J Biochem Cell Biol 31: 489-498, 1999.

460. Van Oort MM, van Doorn JM, Bonen A, Glatz JF, van der Horst DJ, Rodenburg KW, Luiken JJ. Insulin-induced translocation of CD36 to the plasma membrane is reversible and shows similarity to that of GLUT4. Biochim Biophys Acta 1781: 61-71, 2008.

461. Van Oort MM, van Doorn JM, El Hasnaoui M, Glatz JFC, Bonen A, van der Horst DJ, Rodenburg KW, Luiken JJFP. Effects of AMPK activators on the subcellular distribution of fatty acid transporters CD36 and FABPpm. Arch Physiol Biochem 115: 137-146, 2009 .

462. Vila MC, Farese RV. Insulin rapidly increases glycerol-3-phosphate-acyltransferase activity in rat adipocytes. Arch Biochem Biophys 284: 366-368, 1991.

463. Viollet B, Mounier R, Leclerc J, Yazigi A, Foretz M, Andreelli F. Targeting AMP-activated protein kinase as a novel therapeutic approach for the treatment of metabolic disorders. Diabetes Metab 33: 395-402, 2007.

464. Vistisen B, Roepstorff K, Roepstorff C, Bonen A, van Deurs B, Kiens B. Sarcolemmal FAT/CD36 in human skeletal muscle colocalizes with caveolin-3 and is more abundant in type1 than type 2 fibers. J Lipid Res 45: 603-609, 2004.

465. Vorum H, Brodersen R, Kragh-Hansen U, Pedersen AO. Solubility of long-chain fatty acids in phosphate buffer at $\mathrm{pH}$ 7.4. Biochim Biophys Acta 1126: 135-142, 1992.

466. Wang P, Chatham JC. Onset of diabetes in Zucker diabetic fatty (ZDF) rats leads to improved recovery of function after ischemia in the isolated perfused heart. Am J Physiol Endocrinol Metab 286: E725-E736, 2004.

467. Wang PG, Lloyd SG, Zeng H, Bonen A, Chatham JC. The impact of altered substrate utilization on cardiac function in isolated hearts from Zucker diabetic fatty rats. Am J Physiol Heart Circ Physiol 288: H2102-H2110, 2005.

468. Wang Y, Sternfeld L, Yang F, Rodriguez JA, Ross C, Hayden MR, Carriere F, Liu G, Hofer W, Schulz I. Enhanced susceptibility to pancreatitis in severe hypertriglyceridaemic lipoprotein lipase-deficient mice and agonist-like function of pancreatic lipase in pancreatic cells. Gut 58: 422-430, 2009.
469. Watkins PA. Very-long-chain acyl-CoA synthetases. J Biol Chem 283: 1773-1777, 2008.

470. Watkins PA, Lu JF, Braiterman LT, Steinberg SJ, Smith KD. Disruption of a yeast very-long-chain acyl-CoA synthetase gene simulates the cellular phenotype of X-linked adrenoleukodystrophy. Cell Biochem Biophys 32: 333-337, 2000.

471. Watkins PA, Lu JF, Steinberg SJ, Gould SJ, Smith KD, Braiterman LT. Disruption of the Saccharomyces cerevisiae FAT1 gene decreases very long-chain fatty acyl-CoA synthetase activity and elevates intracellular very long-chain fatty acid concentrations. J Biol Chem 273: 18210-18219, 1998.

472. Weimar JD, DiRusso CC, Delio R, Black PN. Functional role of fatty acyl-coenzyme A synthetase in the transmembrane movement and activation of exogenous long-chain fatty acids. $\mathrm{J}$ Biol Chem 277: 29369-29376, 2002.

473. Welsh GI, Leney SE, Lloyd-Lewis B, Wherlock M, Lindsay AJ, McCaffrey MW, Tavare JM. Rip11 is a Rab11- and AS160-RabGAPbinding protein required for insulin-stimulated glucose uptake in adipocytes. J Cell Sci 120: 4197-4208, 2007.

474. Wilkes J, Bonen A, Bell RC. A modified high-fat diet induces insulin resistance in rat skeletal muscle but not adipocytes. $A m J$ Physiol Endocrinol Metab 275: E679-E686, 1998.

475. Williams D, Pessin JE. Mapping of R-SNARE function at distinct intracellular GLUT4 trafficking steps in adipocytes. J Cell Biol 180: 375-387, 2008.

476. Wilmsen HM, Ciaraldi TP, Carter L, Reehman N, Mudaliar SR, Henry RR. Thiazolidinediones upregulate impaired fatty acid uptake in skeletal muscle of type 2 diabetics. Am J Physiol Endocrinol Metab 285: E354-E362, 2003.

477. Winder WW, Hardie DG. Inactivation of acetyl-CoA carboxylase and activation of AMP-activated protein kinase in muscle during exercise. Am J Physiol Endocrinol Metab 270: E299-E304, 1996.

478. Witczak CA, Fujii N, Hirshman MF, Goodyear LJ. $\mathrm{Ca}^{2+} /$ calmodulin-dependent protein kinase kinase-alpha regulates skeletal muscle glucose uptake independent of AMP-activated protein kinase and Akt activation. Diabetes 56: 1403-1409, 2007.

479. Wu Q, Ortegon AM, Tsang B, Doege H, Feingold KR, Stahl A. FATP1 is an insulin-sensitive fatty acid transporter involved in diet-induced obesity. Mol Cell Biol 26: 3455-3467, 2006.

480. Yang J, Sambandam N, Han X, Gross RW, Courtois M, Kovacs A, Febbraio M, Finck BN, Kelly DP. CD36 deficiency rescues lipotoxic cardiomyopathy. Circ Res 100: 1208-1217, 2007.

481. Yoshida Y, Holloway GP, Ljubicic V, Hatta H, Spriet LL, Hood D, Bonen A. Negligible direct lactate oxidation in subsarcolemmal and intermyofibrillar mitochondria obtained from red and white rat skeletal muscle. J Physiol 582: 1317-1335, 2007.

482. Young ME, Guthrie PH, Razeghi P, Leighton B, Abbasi S, Patil S, Youker KA, Taegtmeyer H. Impaired long-chain fatty acid oxidation and contractile dysfunction in the obese Zucker rat heart. Diabetes 51: 2587-2595, 2002.

483. Zeigerer A, McBrayer MK, McGraw TE. Insulin stimulation of GLUT4 exocytosis, but not its inhibition of endocytosis, is dependent on RabGAP AS160. Mol Biol Cell 15: 4406-4415, 2004.

484. Zhou SL, Stump D, Isoal L, Berk PD. Constitutive expression of a saturable transport system for non-esterified fatty acids in Xenopus laevis oocytes. Biochem J 297: 315-319, 1994.

485. Zhou SL, Stump D, Kiang CL, Isola LM, Berk PD. Mitochondrial aspartate aminotransferase expressed on the surface of 3T3-L1 adipocytes mediates saturable fatty acid uptake. Proc Soc Exp Biol Med 208: 263-270, 1995.

486. Zou Z, DiRusso CC, Ctrnacta V, Black PN. Fatty acid transport in Saccharomyces cerevisiae. Directed mutagenesis of FAT1 distinguishes the biochemical activities associated with Fatp1. J Biol Chem 277: 31062-31071, 2002.

487. Zou Z, Tong F, Faergeman NJ, Borsting C, Black PN, DiRusso CC. Vectorial acylation in Saccharomyces cerevisiae. Fat1p and fatty acyl-CoA synthetase are interacting components of a fatty acid import complex. J Biol Chem 278: 16414-16422, 2003. 